\title{
Liver substrate metabolism in non-alcoholic fatty liver
}

Citation for published version (APA):

Roumans, K. H. M. (2021). Liver substrate metabolism in non-alcoholic fatty liver: role of hepatic lipid composition and hepatic glycogen measured by MR-techniques. [Doctoral Thesis, Maastricht University]. Gildeprint Drukkerijen. https://doi.org/10.26481/dis.20210915kr

Document status and date:

Published: 01/01/2021

DOI:

10.26481/dis.20210915kr

Document Version:

Publisher's PDF, also known as Version of record

\section{Please check the document version of this publication:}

- A submitted manuscript is the version of the article upon submission and before peer-review. There can be important differences between the submitted version and the official published version of record.

People interested in the research are advised to contact the author for the final version of the publication, or visit the DOI to the publisher's website.

- The final author version and the galley proof are versions of the publication after peer review.

- The final published version features the final layout of the paper including the volume, issue and page numbers.

Link to publication

\footnotetext{
General rights rights.

- You may freely distribute the URL identifying the publication in the public portal. please follow below link for the End User Agreement:

www.umlib.nl/taverne-license

Take down policy

If you believe that this document breaches copyright please contact us at:

repository@maastrichtuniversity.nl

providing details and we will investigate your claim.
}

Copyright and moral rights for the publications made accessible in the public portal are retained by the authors and/or other copyright owners and it is a condition of accessing publications that users recognise and abide by the legal requirements associated with these

- Users may download and print one copy of any publication from the public portal for the purpose of private study or research.

- You may not further distribute the material or use it for any profit-making activity or commercial gain

If the publication is distributed under the terms of Article $25 \mathrm{fa}$ of the Dutch Copyright Act, indicated by the "Taverne" license above, 
Liver substrate metabolism in non-alcoholic fatty liver:

role of hepatic lipid composition and hepatic glycogen measured by MR-techniques

Kay H.M. Roumans 


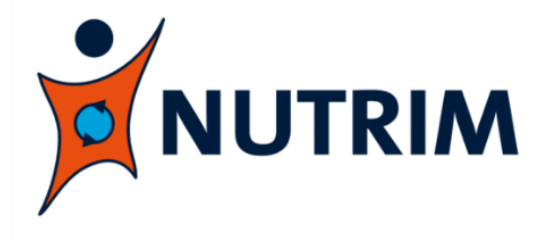

\section{Health Holland $\quad \forall$ tolland.}

The research presented in this thesis was performed within NUTRIM School of Nutrition and Translational Research in Metabolism. The research described in this thesis was conducted within the Health Holland framework and was financially supported by Unilever R\&D Wageningen and Health Holland. Chapter 7 was supported by a grant of the Dutch Heart Foundation (CVON2014-02).

Printed by: Gildeprint - www.gildeprint.nl

ISBN: 9789464192582

Copyright (C) Kay Roumans, Maastricht 2021 


\title{
Liver substrate metabolism in non-alcoholic fatty liver:
}

role of hepatic lipid composition and hepatic glycogen measured by MR-techniques

\author{
DISSERTATION
}

to obtain the degree of Doctor at the Maastricht University, on the authority of the Rector Magnificus,

Prof. dr. Rianne M. Letschert

in accordance with the decision of the Board of Deans, to be defended in public on Wednesday 15 September 2021, at 16.00 hours

by

Kay Henricus Marie Roumans 


\section{Supervisor:}

Prof. dr. P. Schrauwen

\section{Co-supervisors:}

Dr. V. Schrauwen-Hinderling

Dr. ir. L. Lindeboom, Imec - High Tech Campus Eindhoven

\section{Assessment Committee:}

Prof. dr. J. Plat (chair)

Prof. dr. L. Hodson, University of Oxford, United Kingdom

Prof. dr. E. Kooi

Prof. dr. S. La Fleur, Amsterdam UMC

Dr. R. Meex 


\section{TABLE OF CONTENTS}

CHAPTER $1 \quad$ General introduction and outline 7

CHAPTER 2 Liver fat storage and disposal pathways: methodologies, contributions to liver fat accumulation and dietary effects

CHAPTER 3 Hepatic saturated fatty acid fraction is associated with de novo lipogenesis and hepatic insulin resistance

CHAPTER 4 Hepatic glycogen is not modulated by one night of prolonged fasting in people with non-alcoholic fatty liver

CHAPTER 5 Effect of a low compared to a high glycemic index and saturated fat diet on liver fat and substrate metabolism

CHAPTER 6 Hepatic water and lipid T2 relaxation times are associated with level of steatosis - implications for absolute fat quantification

CHAPTER 7 Nicotinamide riboside supplementation alters body composition and skeletal muscle acetylcarnitine concentrations in healthy obese humans

CHAPTER 8 General discussion and conclusion

Addendum 185

Impact 186

Summary 189

Samenvatting 192

About the author 195

List of publications 196

Dankwoord 197 

CHAPTER 1

General introduction and outline 


\section{Non-alcoholic fatty liver}

Obesity and overweight are a major worldwide problem. Worldwide obesity rates have tripled in the last 45 years (1). In 2016, 13\% of the adults were obese and $40 \%$ of the adults were overweight (1). Obesity and overweight are characterized by excessive fat storage in adipose tissue. However, fat is also stored in non-adipose tissue, such as in liver, muscle and heart. Such fat storage is referred to as ectopic fat storage. In the liver, fat can be stored in hepatocytes and when intrahepatic lipids exceed $5 \%$ of liver weight, in absence of excessive alcohol consumption, this is defined as non-alcoholic fatty liver (NAFL). Currently, NAFL is the leading cause of chronic liver disease and it is estimated that $25 \%$ of the worldwide population has NAFL (2). Moreover, NAFL is even more prevalent in obesity, with rates estimated as high as $55-75 \%(3,4)$. NAFL can progress to more severe stages of liver disease, such as non-alcoholic steatohepatitis (NASH) and liver cirrhosis, conditions in which liver function is severely hampered and the risk for hepatocellular carcinoma is increased. Importantly, NAFL per se is already very strongly associated with metabolic diseases such as cardiovascular disease (CVD) and type II diabetes (T2D) (5-7). As obesity is still on the rise and NAFL prevalence, together with its metabolic consequences, is expected to increase even further, it will have a tremendous clinical and economic impact (2). Knowledge on how NAFL contributes to cardiometabolic disease is limited, particularly because of the limitations in techniques available to study this relationship non-invasively. Thus, advanced methodologies are needed to investigate metabolic processes in NAFL.

\section{Advanced MR methodologies to assess metabolic processes in NAFL}

Non-invasive imaging techniques, such as magnetic resonance spectroscopy (MRS) can be applied to acquire metabolic information. MRS yields chemical information of tissues, thereby making it a useful technique to study substrate stores and dynamics in different organs. As such, MRS has been shown to be of great value in determining ectopic lipid accumulation. In the liver, ${ }^{1} \mathrm{H}-\mathrm{MRS}$ is the gold standard to determine fat content. Using ${ }^{1} \mathrm{H}$ $M R S$, a spectrum is generated from which the lipid and water resonances can be quantified and from these resonances, intrahepatic lipid (IHL) content can be calculated. This technique has been used frequently in human studies, showing that IHL stores relate to hepatic insulin resistance $(8,9)$ and are increased in patients with type 2 diabetes $(10)$ and furthermore, change upon exercise (11-13) and dietary (14-17) interventions. Apart from determining the total amount of fat stored in the liver, ${ }^{1} \mathrm{H}$-MRS could also be used to get more qualitative information of stored IHL (i.e. type of fatty acids; saturated (SFA), monounsaturated (MUFA), poly-unsaturated fatty acids (PUFA)). Determining the hepatic fatty acid fractions is however challenging as the resonance of the unsaturated fatty acids are 
small, resonances are overlapping and imperfect suppression of the large water resonance can lead to contamination of other resonances. So far, parameters linked to the degree of unsaturation have been measured in some studies (18-21). These parameters indicate the relative abundance of double bonds in the fatty acid signal, however they do not specifically and robustly differentiate between hepatic SFA, MUFA and PUFA fractions. Such separation in SFA, MUFA and PUFA can yield interesting metabolic information as it is suggested that specifically SFA may have detrimental effects on metabolic health and the nature of fatty acid can also be indicative of the source of the hepatic fat. Other nuclei that can be investigated using MRS include ${ }^{13} \mathrm{C}$. Direct ${ }^{13} \mathrm{C}$-MRS has been applied on the liver in the past and can be used to determine hepatic glycogen stores. Studies have applied this method showing reductions in hepatic glycogen during the fasting period (22-24), postprandial increases in hepatic glycogen $(23,25)$ and defects in hepatic glycogen metabolism in patients with type 2 diabetes (23). Furthermore, hepatic glycogen has been shown to change upon dietary changes in glycaemic index (16). Presently, very little is known about glycogen dynamics in NAFL, even though it may be highly relevant in hepatic substrate partitioning. These advanced MRS techniques can be applied to investigate hepatic substrate metabolism and to better understand the metabolic changes that take place during the development of NAFL.

\section{Thesis outline}

The aim of this thesis is to investigate liver substrate metabolism in NAFL using advanced MR methodology. In chapter $\mathbf{2}$ the techniques available to study the different pathways leading to $\mathrm{IHL}$ accumulation are reviewed. Furthermore, studies using these techniques to estimate the relative contribution of the different pathways to IHL accumulation or to study dietary modulation of these pathways are discussed. De novo lipogenesis (DNL) is one of these pathways and represents the synthesis of fatty acids from carbohydrates. DNL is found to be strongly increased in NAFL and may be one of the underlying pathways leading to NAFL. The end product of DNL is SFA, and therefore high DNL rates might contribute to an increased hepatic SFA fraction. It is suggested that specifically these SFA negatively influence metabolic health. Therefore, in chapter $\mathbf{3}$ a novel magnetic resonance technique is developed, validated and applied, that enabled non-invasively quantification of the fractions of hepatic SFA, MUFA and PUFA separately, in healthy and metabolically compromised human volunteers. Using this methodology, the hypothesis that higher rates of DNL are associated with an increased fraction of SFA in human liver is tested. Additionally, it is investigated if populations at higher risk to develop metabolic complications are 
characterized by altered hepatic fatty acid composition, and whether hepatic fatty acid composition is related to hepatic insulin sensitivity.

Hepatic lipid- and carbohydrate metabolism are strongly intertwined and a key factor in determining substrate metabolism and partitioning towards oxidation or storage may be hepatic glycogen. Glycogen represents a dynamic local carbohydrate store and is usually thought to change dynamically over 24 hours; decreasing during fasting and being replenished upon refeeding. The fasting-induced glycogen depletion is generally thought of being a healthy reflection of metabolic flexibility and it is suggested that the regular depletion of hepatic glycogen stores may be underlying the beneficial effects seen with time restricting eating regimes. To study the importance of hepatic glycogen stores in NAFL, prolonged overnight fasting is used as a tool to modulate hepatic glycogen in volunteers with NAFL in chapter 4 . In this chapter it is investigated whether prolonged fasting would acutely lower hepatic glycogen and thereby, improve substrate oxidation during the night and postprandially in volunteers with NAFL. Additionally, effects of 5 days prolonged fasting on liver fat content and composition are studied.

In chapter $\mathbf{5}$ it is investigated whether changing the degree of fat saturation and the glycemic index (GI) in the diet could influence IHL content. Human studies investigating the effect of dietary fat and carbohydrate type on IHL are limited to proof of principle studies with exaggerated differences in diet composition. Therefore, two two-week diets differing in GI and SFA content are designed to study the effect of changes in dietary type of carbohydrate and fat, while still matching dietary patterns of the general population. Furthermore, it is studied whether a low vs. high GI/SFA diet increased whole-body fat oxidation, decreased glycemic response and reduced hepatic glycogen levels.

Chapter 6 focusses on the importance of different factors that could influence IHL quantification. Specifically, it is evaluated how variations in $\mathrm{T} 2$ relaxation times affect the calculations of absolute $\mathrm{IHL} \%(\mathrm{w} / \mathrm{w})$ and to what extend the bias occurs while using different IHL quantification formulas. In chapter 7 advanced techniques are applied to assess the metabolic effect of six-week nicotamine riboside (NR) supplementation. Results from animal studies suggest that NR, as an NAD+ precursor, can have metabolically beneficial effects, counteracting the negative effects of overweight and aging, however, human data is largely lacking. Therefore, insulin sensitivity and IHL content are investigated by hyperinsulinemic-euglycemic clamp technique and MRS respectively after NR and placebo. Finally, in chapter $\mathbf{8}$ the main results and conclusions of the previous chapters in this thesis are discussed in a broader perspective. Furthermore, future directions in the field of liver substrate metabolism are discussed. 


\section{REFERENCES}

1. WHO. Obesity and overweight 2020 [Available from: https://www.who.int/newsroom/fact-sheets/detail/obesity-and-overweight.

2. Younossi Z, Tacke F, Arrese M, Chander Sharma B, Mostafa I, Bugianesi E, et al. Global Perspectives on Nonalcoholic Fatty Liver Disease and Nonalcoholic Steatohepatitis. Hepatology (Baltimore, Md). 2019;69(6):2672-82.

3. Gaggini M, Morelli M, Buzzigoli E, DeFronzo RA, Bugianesi E, Gastaldelli A. Nonalcoholic fatty liver disease (NAFLD) and its connection with insulin resistance, dyslipidemia, atherosclerosis and coronary heart disease. Nutrients. 2013;5(5):1544-60.

4. Bellentani S, Saccoccio G, Masutti F, Croce LS, Brandi G, Sasso F, et al. Prevalence of and risk factors for hepatic steatosis in Northern Italy. Annals of internal medicine. 2000;132(2):112-7.

5. Lim S, Oh TJ, Koh KK. Mechanistic link between nonalcoholic fatty liver disease and cardiometabolic disorders. International journal of cardiology. 2015;201:408-14.

6. Targher G, Day CP, Bonora E. Risk of cardiovascular disease in patients with nonalcoholic fatty liver disease. The New England journal of medicine. 2010;363(14):1341-50.

7. Targher G, Byrne CD. Clinical Review: Nonalcoholic fatty liver disease: a novel cardiometabolic risk factor for type 2 diabetes and its complications. The Journal of clinical endocrinology and metabolism. 2013;98(2):483-95.

8. Kotronen A, Vehkavaara S, Seppälä-Lindroos A, Bergholm R, Yki-Järvinen H. Effect of liver fat on insulin clearance. American journal of physiology Endocrinology and metabolism. 2007;293(6):E1709-15.

9. Brouwers B, Schrauwen-Hinderling VB, Jelenik T, Gemmink A, Havekes B, Bruls Y, et al. Metabolic disturbances of non-alcoholic fatty liver resemble the alterations typical for type 2 diabetes. Clinical science (London, England : 1979). 2017;131(15):1905-17.

10. Kotronen A, Juurinen L, Tiikkainen M, Vehkavaara S, Yki-Järvinen H. Increased liver fat, impaired insulin clearance, and hepatic and adipose tissue insulin resistance in type 2 diabetes. Gastroenterology. 2008;135(1):122-30.

11. Brouwers B, Schrauwen-Hinderling VB, Jelenik T, Gemmink A, Sparks LM, Havekes $B$, et al. Exercise training reduces intrahepatic lipid content in people with and people without nonalcoholic fatty liver. American journal of physiology Endocrinology and metabolism. 2018;314(2):E165-e73.

12. Johnson NA, Sachinwalla T, Walton DW, Smith K, Armstrong A, Thompson MW, et al. Aerobic exercise training reduces hepatic and visceral lipids in obese individuals without weight loss. Hepatology (Baltimore, Md). 2009;50(4):1105-12.

13. Sullivan S, Kirk EP, Mittendorfer B, Patterson BW, Klein S. Randomized trial of exercise effect on intrahepatic triglyceride content and lipid kinetics in nonalcoholic fatty liver disease. Hepatology (Baltimore, Md). 2012;55(6):1738-45.

14. van Herpen NA, Schrauwen-Hinderling VB, Schaart G, Mensink RP, Schrauwen P. Three weeks on a high-fat diet increases intrahepatic lipid accumulation and 
decreases metabolic flexibility in healthy overweight men. The Journal of clinical endocrinology and metabolism. 2011;96(4):E691-5.

15. Luukkonen PK, Sädevirta S, Zhou Y, Kayser B, Ali A, Ahonen L, et al. Saturated Fat Is More Metabolically Harmful for the Human Liver Than Unsaturated Fat or Simple Sugars. Diabetes care. 2018;41(8):1732-9.

16. Bawden S, Stephenson M, Falcone Y, Lingaya M, Ciampi E, Hunter K, et al. Increased liver fat and glycogen stores after consumption of high versus low glycaemic index food: A randomized crossover study. Diabetes, obesity \& metabolism. 2017;19(1):70-7.

17. Bjermo H, Iggman D, Kullberg J, Dahlman I, Johansson L, Persson L, et al. Effects of n-6 PUFAs compared with SFAs on liver fat, lipoproteins, and inflammation in abdominal obesity: a randomized controlled trial. The American journal of clinical nutrition. 2012;95(5):1003-12.

18. Johnson NA, Walton DW, Sachinwalla T, Thompson CH, Smith K, Ruell PA, et al. Noninvasive assessment of hepatic lipid composition: Advancing understanding and management of fatty liver disorders. Hepatology (Baltimore, Md). 2008;47(5):1513-23.

19. Hamilton G, Yokoo T, Bydder M, Cruite I, Schroeder ME, Sirlin CB, et al. In vivo characterization of the liver fat (1)H MR spectrum. NMR in biomedicine. 2011;24(7):784-90.

20. Lundbom J, Hakkarainen A, Soderlund S, Westerbacka J, Lundbom N, Taskinen MR. Long-TE $1 \mathrm{H}$ MRS suggests that liver fat is more saturated than subcutaneous and visceral fat. NMR in biomedicine. 2011;24(3):238-45.

21. Gajdosik M, Chadzynski GL, Hangel G, Mlynarik V, Chmelik M, Valkovic L, et al. Ultrashort-TE stimulated echo acquisition mode (STEAM) improves the quantification of lipids and fatty acid chain unsaturation in the human liver at $7 \mathrm{~T}$. NMR in biomedicine. 2015;28(10):1283-93.

22. Magnusson I, Rothman DL, Katz LD, Shulman RG, Shulman GI. Increased rate of gluconeogenesis in type II diabetes mellitus. A $13 \mathrm{C}$ nuclear magnetic resonance study. The Journal of clinical investigation. 1992;90(4):1323-7.

23. Krssak M, Brehm A, Bernroider E, Anderwald C, Nowotny P, Dalla Man C, et al. Alterations in postprandial hepatic glycogen metabolism in type 2 diabetes. Diabetes. 2004;53(12):3048-56.

24. Macauley M, Smith FE, Thelwall PE, Hollingsworth KG, Taylor R. Diurnal variation in skeletal muscle and liver glycogen in humans with normal health and Type 2 diabetes. Clinical science (London, England : 1979). 2015;128(10):707-13.

25. Taylor R, Magnusson I, Rothman DL, Cline GW, Caumo A, Cobelli C, et al. Direct assessment of liver glycogen storage by $13 \mathrm{C}$ nuclear magnetic resonance spectroscopy and regulation of glucose homeostasis after a mixed meal in normal subjects. The Journal of clinical investigation. 1996;97(1):126-32. 
General introduction 


\section{CHAPTER 2}

\section{Liver fat storage and disposal pathways: methodologies, contributions to liver fat accumulation and dietary effects}

K.H.M. Roumans ${ }^{1}$, J. Basset Sagarminaga ${ }^{1}$, H.P.F. Peters ${ }^{2}$, P. Schrauwen ${ }^{1}$, V.B. Schrauwen-Hinderling ${ }^{1,3}$.

${ }^{1}$ Department of Nutrition and Movement Sciences, Maastricht University,

P.O. BOX 616, 6200 MD, Maastricht, The Netherlands.

${ }^{2}$ Unilever Food Innovation Center, Plantage 14, 6708 WJ Wageningen, The Netherlands.

${ }^{3}$ Department of Radiology and Nuclear Medicine, Maastricht University Medical Center, P.O. BOX 5800, 6202 AZ, Maastricht, The Netherlands.

This chapter has been published in slightly adjusted form:

Curr Opin Lipidol. 2021 Feb 1;32(1):9-15 


\section{ABSTRACT}

Non-alcoholic fatty liver (NAFL) is the most common liver disorder in western society and is strongly associated with metabolic diseases such as cardiovascular disease and type II diabetes. This condition of excessive liver fat storage is the result of an imbalance between lipid storage (direct storage of fat from a meal, de novo lipogenesis and uptake of nonesterified fatty acids mainly derived from adipose tissue lipolysis) and disposal (oxidation and secretion). Knowledge on the contribution of each of these pathways to liver fat content in humans is essential in order to develop strategies to prevent and treat NAFL. Techniques used to assess these individual pathways are predominantly stable isotope and radioisotope techniques, which require specific expertise and are costly. Validated indirect markers that are easier to apply, are often lacking. These methodological limitations also translate into a limited amount of studies that have been performed to investigate to what extent the different accumulation and disposal routes can be modulated by diet. Here, we review the techniques available to study the different pathways leading to liver fat accumulation. Furthermore, we will review studies using these techniques to estimate the relative contribution of the different pathways to liver fat accumulation or to study dietary modulation of these pathways. 


\section{INTRODUCTION}

A dramatic rise in the prevalence of non-alcoholic fatty liver (NAFL) has been observed over the last few decades and it is now considered to be the most common liver disorder worldwide (1). NAFL is characterized by excessive fat accumulation in the liver that is not associated with high alcohol consumption. NAFL can progress to more severe stages of liver disease, such as non-alcoholic steatohepatitis (NASH) and liver cirrhosis, conditions in which liver function is severely hampered and the risk for hepatocellular carcinoma is increased. Importantly, even if no further progression of liver disease occurs, NAFL per se is also very strongly associated with metabolic diseases such as cardiovascular disease (CVD) and type II diabetes (T2D) (2-4). Strikingly, the prevalence of NAFL in obese people is estimated to be as high as $55-75 \%(5,6)$. Therefore, reducing liver fat content is a promising target to decrease the risk of these metabolic diseases.

Excessive fat accumulation in the liver is thought to be the result of an imbalance between lipid storage (due to increased delivery and synthesis), and disposal. It is now well established that fat that is stored in the liver (in hepatocytes) originates from three main sources: 1) direct fat storage from a meal, 2) de novo synthesis of fatty acids from glucose, fructose or amino acids (de novo lipogenesis; DNL) 3) from uptake of plasma non-esterified fatty acids (NEFA) mainly derived from adipose tissue lipolysis (figure 1). In healthy individuals, these processes are balanced by the activation of metabolic pathways that contribute to the mobilization and clearance of hepatic fat. These pathways include: 1) mitochondrial fatty acid oxidation for the production of ATP and fatty acid shuttling towards ketogenesis after initial ß-oxidation (acetyl-CoA disposal), 2) fatty acid incorporation into VLDL-particles to be secreted into the circulation (figure 1). While the pathways leading to hepatic fat accumulation have long been identified, knowledge on the contribution of each of these pathways to liver fat content in humans is sparse, in part because appropriate techniques are lacking. Gaining a better understanding of the mechanisms, which contribute to hepatic fat accumulation is crucial to the development of effective treatment strategies for NAFL and its associated metabolic disturbances.

In this review, an overview of the techniques available to study the different pathways leading to liver fat accumulation will be provided. Furthermore, we will review studies using these techniques to estimate the relative contribution of the different pathways to liver fat accumulation or to study dietary modulation of these pathways. 


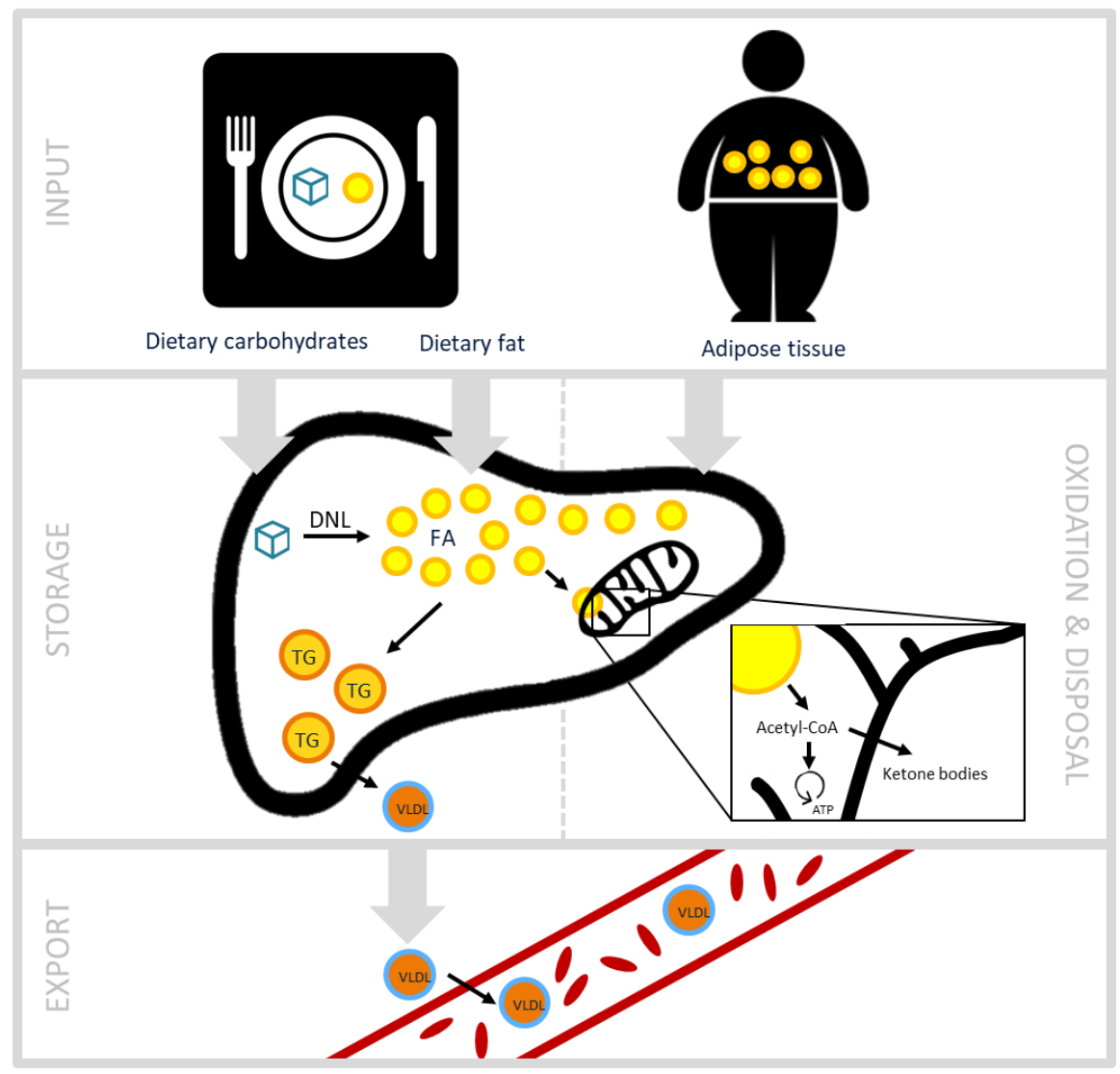

Figure 1: Overview of liver fat storage and disposal pathways. Storage pathways include direct fat storage from a meal, de novo lipogenesis (DNL) from carbohydrates and adipose-tissue derived non-esterified fatty acids (NEFA) uptake. Liver lipid disposal pathways are mitochondrial fatty acid (FA) oxidation and ketogenesis after initial ßoxidation (acetyl-CoA disposal), and triglyceride (TG) incorporation into very low density lipid (VLDL)-particles to be secreted into the circulation.

\section{DIETARY FAT STORAGE}

\subsection{Pathway description}

Following a meal, dietary fat is taken up in the intestines (7). In the enterocytes chylomicrons (CM) are formed. These large complexes of triglycerides (TG), phospholipids, cholesterol and proteins enter the circulation via the lymphatic system (7). The CM particles are usually too big to pass the fenestrations of hepatic sinusoids and will not reach the surface of the hepatocyte (8). Therefore, the particles first need to be 'cleared' by lipoprotein lipase (LPL)-mediated hydrolysis in muscle and adipose tissue. As a result, CM 
lose TG content and remnant particles are formed. These remnant particles, in contrast to $\mathrm{CM}$ itself, can penetrate the fenestrations, reach the surface of hepatocytes and can be taken up in the hepatocytes through receptor-mediated uptake where they are cleared of their remaining TG (8). Another route by which dietary fat reaches the liver is by so-called fatty acid spill-over: the fatty acids that are released by LPL-mediated lipolysis at the endothelium in adipose tissue or muscle will not all be taken up by the peripheral tissues, but in part remain in the circulation and thereby feed into the plasma NEFA pool (9). Subsequently, the liver can also take up these 'spill-over fatty acids' mediated by fatty acid transport proteins (10).

\subsection{Techniques to measure this pathway}

\subsubsection{Stable isotope measurements in plasma}

Dietary fat uptake is commonly measured by using fatty acid tracers, for example, fatty acids that are enriched with the stable carbon isotope ${ }^{13} \mathrm{C}(11-14)$ or deuterium $\left({ }^{2} \mathrm{H}\right)(15-18)$. Carbon-13 and deuterium have a low natural abundance ( $1.1 \%$ and $0.015 \%$, respectively), making them good candidates for tracer studies focusing on dietary fat uptake. Usually, a meal with ${ }^{13} \mathrm{C}$-labeled palmitate, deuterated tri-palmitate or $\left[{ }^{2} \mathrm{H}_{35}\right]$ stearate is given to trace incorporation of meal fat in very low-density lipoprotein TG (VLDL-TG) (11-18). The fatty acid composition and the tracer enrichment were shown to be similar in VLDL-TG and liver TG (determined from liver biopsies), therefore the ${ }^{13} \mathrm{C}$ and ${ }^{2} \mathrm{H}$ enrichment of plasma VLDLTGs can be used as a surrogate for liver fat enrichment and can be used to determine hepatic storage of meal fat $(15,19)$. Isotopic enrichments in VLDL-TG are generally determined by GC/MS.

\subsubsection{Spectroscopy measurements in the liver}

To investigate tracer enrichments directly in the liver, liver biopsies have been used (15). An alternative approach is through spectroscopy or PET methodology, assessing which proportion of the lipids in a meal is ending up in the liver. Magnetic resonance spectroscopy (MRS) techniques can be used to measure ${ }^{13} \mathrm{C}$ enrichment directly in the liver after consumption of ${ }^{13} \mathrm{C}$-labeled fatty acids $(20,21)$. The difficulty with standard ${ }^{13} \mathrm{C}-\mathrm{MRS}$ is the low sensitivity of this method and the high contamination of the ${ }^{13} \mathrm{C}$ lipid spectra of the liver by ${ }^{13} \mathrm{C}$ signal from adipose tissue, which is due to suboptimal localization of the measured signal to the liver. To overcome these problems, so called ${ }^{13} \mathrm{C}$-edited methods can be used. With this method, the superior sensitivity and localization of ${ }^{1} \mathrm{H}-\mathrm{MRS}$ can be used by applying ${ }^{1} \mathrm{H}$-MRS techniques that specifically quantify the signal of ${ }^{1} \mathrm{H}$ nuclei that are directly 
linked to ${ }^{13} \mathrm{C}$ and therefore, the ${ }^{1} \mathrm{H}$-MRS signal becomes proportional of ${ }^{13} \mathrm{C}$ enrichment ('indirect' ${ }^{13} \mathrm{C}$ spectroscopy or ${ }^{13} \mathrm{C}$-edited ${ }^{1} \mathrm{H}$-MRS). Indeed, it was shown that such indirect ${ }^{13} \mathrm{C}$ spectroscopy can be used to quantify the ${ }^{13} \mathrm{C}$ lipid signal in the liver and thereby 'track' the ${ }^{13} \mathrm{C}$-fatty acids originating from a meal (21). Since the ${ }^{13} \mathrm{C}$ signal is followed over time in the liver, the measured ${ }^{13} \mathrm{C}$ signal in the liver reflects net storage of dietary fat (uptake minus disposal), also referred to as dietary fat retention.

\subsubsection{Radioisotopic measurements in the liver}

Another technique that has been used to determine dietary fat uptake in the liver is positron emission tomography (PET) in combination with oral intake of $14(\mathrm{R}, \mathrm{S})$-[(18)F]fluoro-6-thiaheptadecanoic acid ( $\left.{ }^{18} \mathrm{FTHA}\right)$ tracer, a long chain fatty acid analog containing ${ }^{18}$ fluor, which can be detected by PET (22). The radioactive signal of this tracer can be measured in time and in different target organs, including the liver. ${ }^{18} \mathrm{FTHA}$ cannot be metabolized after entering the organs, but can be esterified and incorporated in protein complexes. Therefore, in tissues like skeletal muscle or the heart ${ }^{18} \mathrm{FTHA}$ is trapped in the tissue and reflects fat uptake only, while in the liver, this tracer can also leave the organ when secreted in VLDL, similar to the recycling of dietary fatty acids in VLDL. Therefore, it reflects the balance between uptake and export, where oxidation is not considered (22).

\subsection{Contribution to liver fat accumulation and influence of diet}

Generally, dietary fat storage in the liver is thought to be small. Using deuterated tripalmitate as a tracer in whole-food meals, the percentage of VLDL-TG contributed by dietary fat over four days in NAFL patients was estimated to be $15 \%$ (15). Less physiologically relevant, oral and duodenal administration of liquid food has been used to determine contribution in healthy subjects, finding values of around $15-20 \%$ and $10-15 \%$, respectively five hours after a meal (16). Using indirect ${ }^{13} \mathrm{C}$ spectroscopy, postprandial retention of orally administered ${ }^{13} \mathrm{C}$-labeled fatty acids in the liver after 6 hours has been found to be $1.5 \%$, for both lean and obese subjects (21). This equals dietary fat making up around $10 \%$ of the total liver fat pool per day for an individual with a relatively low liver fat percentage (21). Also between glucose tolerant and glucose intolerant subjects no differences in dietary fat uptake have been reported upon a standardized mixed meal, when measuring between 90 and 120 minutes after oral administration of ${ }^{18} \mathrm{FTHA}$ tracer (22).

A study performed by Chong et al. showed by using oral administration of $\left[\mathrm{U}-{ }^{13} \mathrm{C}\right]$ palmitate that dietary fat contribution to VLDL-TG was similar upon a three-day high-fat and highcarbohydrate diet in eight healthy volunteers (around 15\% six hours after a mixed meal) 
(11). Using the same tracer, Parry et al. showed that there was no difference in dietary fat contribution to VLDL-TG upon a 4-week diet enriched with saturated fat compared to a diet enriched with free sugars in sixteen overweight males, with values around $5-10 \%$ six hours after a meal (14). In addition, supplementation with omega-3 fatty acids (4 g/day eicosapentaenoic acid (EPA) + docosahexaenoic acid (DHA) as ethyl esters) for eight weeks in thirty-eight healthy men did also not change dietary fat retention six hours after a meal compared to baseline (13). With respect to the type of carbohydrates in a meal, [U${ }^{13} \mathrm{C}$ ]palmitate experiments have shown in sixteen healthy volunteers that there is lower relative contribution of dietary fatty acids to VLDL-TG after consumption of a high fructose/low glucose compared to a low fructose/high glucose meal (23). To date, spectroscopy and PET methods have not been used to investigate the impact of different diet compositions on dietary fat retention.

Altogether, data on the importance of dietary fat retention in the liver is limited and measured in different populations and different diets. Based on a few studies, it seems that the relative contribution of meal-derived fat storage is rather robust. However, the potential impact of carbohydrate type and fat type is interesting to investigate in more detail.

\section{DE NOVO LIPOGENESIS}

\subsection{Pathway description}

De novo lipogenesis (DNL) is another pathway contributing to the accumulation of fat in the liver. This pathway comprises reactions in the mitochondrial matrix and the cytosol of hepatocytes that lead to the formation of fatty acids (24). Acetyl-CoA, derived from catabolic pathways of carbohydrates or amino acids, serves as the main substrate for this process (24). The cytosolic acetyl-CoA will be converted to the saturated fatty acid palmitate through several enzymatic reactions involving the enzymes Acetyl-CoA carboxylase (ACC), which forms malonyl-CoA from acetyl-CoA, and fatty acid synthase (FAS), which in turn synthesizes palmitate from malonyl-CoA (10).

\subsection{Techniques to measure this pathway}

\subsection{1 ${ }^{13} \mathrm{C}$-acetate infusion}

${ }^{13} \mathrm{C}$-acetate has frequently been used in studies to determine DNL contribution to liver fat. Upon intravenous infusion, this labeled acetate will be taken up by the liver. In hepatocytes, the acetate is converted to acetyl-CoA, thereby labeling the intrahepatic acetyl-CoA pool. 
This ${ }^{13} \mathrm{C}$ labeled acetyl-CoA is then converted to palmitate by the enzymes ACC and FAS. Based on tracer enrichments in the intrahepatic acetyl-CoA precursor pool and the product pool of VLDL-palmitate, fractional synthesis of fatty acids can be determined from the precursor to product ratio, however this requires taking hepatic biopsies for determination of acetyl-CoA precursor pool enrichment (25). To overcome this, mass isotopomer distribution analysis (MIDA) has been introduced by Hellerstein and Neese (26). Through this technique, the acetyl-CoA precursor enrichment can be determined from the relative distribution pattern of labeled isotopomers in the product (VLDL-palmitate).

Most studies using this tracer have used short infusion protocols ranging from several hours to two days (16-18, 27-34). However, infusion protocols of more than two days have also been used (15), improving the assessment of DNL contribution to liver fat in people with larger hepatic lipid pools: since the intrahepatic lipid pool in NAFL takes days or even weeks to turnover, short-labeling protocols may underestimate the true DNL contribution (35). A disadvantage of longer protocols, however, is that with stable isotope approaches there will be recycling of isotope labeled fatty acids that can interfere with the determination of DNL.

\subsubsection{Deuterium oxide measurements}

Another frequently used stable isotope tracer for DNL measurement is deuterium oxide (12, $13,23,36-41)$. Upon oral administration, the body water pool will become enriched in deuterium. Consequently, deuterium will also be incorporated in NADPH, a metabolite that is used in the last step of the DNL pathway for the de novo synthesis of palmitate, thus labeling the palmitate formed in DNL (42). Compared to the intravenous infusion of ${ }^{13} \mathrm{C}$ acetate, the use of deuterium oxide is less demanding as this tracer can be easily consumed orally as enriched water.

Similar to ${ }^{13} \mathrm{C}$ infusion protocols, deuterium tracer protocols largely differ in duration, varying from several hours to up to 10 days $(12,36)$. Most frequently, a short protocol is used, consisting of a loading dose of deuterium labeled water provided in two servings in the evening, followed by ad libitum intake of low-dose deuterium labeled water to maintain body water enrichment levels $(13,36-38,41)$. In case of applying a deuterium protocol over up to 10 days, with oral intake of deuterium oxide at home, it should be noted that standardization of participant's lifestyle over these days is very important to limit unwanted lifestyle influences. 


\subsubsection{Plasma markers of DNL}

Besides the use of stable isotope tracers, plasma FA levels/ratios are often used to infer hepatic DNL. The main plasma markers used are the lipogenic index, the percentage increase in palmitate ('new palmitate'), and the Stearoyl-CoA desaturase (SCD) index. In large scale studies, where more costly and time-consuming techniques would not be feasible, these indices can be used as an alternative marker for tracer-based methods.

The lipogenic index $(11,33,43-45)$ is expressed as the ratio of palmitate $(16: 0)$ to linoleate $(18: 2 n 6)$ in VLDL-TG, and has been shown to be in agreement with ${ }^{13} \mathrm{C}$ labeled acetate measurements following a high carbohydrate diet (33). As the primary end product of DNL is saturated fatty acids in humans, increased DNL rates will lead to a higher relative contribution of palmitate to VLDL-TG mainly at the expense of PUFAs such as linoleate (32). The body cannot synthesize linoleate and therefore its appearance in plasma can only be from diet and adipose tissue. By matching the proportion of linoleate in the diet to the proportion in adipose tissue or assuming that linoleate in adipose tissue and diet are similar, linoleate contribution to VLDL-TG reflects the contribution of fatty acids not synthesized by the body and its dilution reflects DNL (33).

Instead of expressing palmitate relatively to linoleate, determining the percentage increase in only palmitate has also been used to measure DNL (46). The relative increase in palmitate contribution to VLDL-TG in response to fructose (and glucose) feeding has been shown to highly correlate with the change in DNL as determined by ${ }^{13} \mathrm{C}$ labeled acetate infusion combined with MIDA (46).

As isotope tracer studies are more costly and more challenging to perform, using these two surrogate markers of DNL could be a good alternative for large-scale studies. Important to note is that these markers should be used within the defined feeding conditions they are designed for, namely high simple carbohydrate and fructose feeding, as recently it has been shown that the lipogenic index poorly reflects DNL in habitual diet conditions (47). This is likely due to the significant effect that dietary fat intake can have on the lipid composition, and thereby also palmitate content, of VLDL-TG.

Next to the lipogenic index and increase in palmitate, the $\mathrm{SCD} 1_{(16)}$ index has been proposed as a marker for DNL $(11,48)$. SCD1 is a key enzyme involved in lipogenesis as it converts SFAs (mainly palmitic and stearic acid) to MUFAs. The ratio of $16: 1 \mathrm{n}-7$ to $16: 0$ [SCD1 $1_{(16)}$ ] is often used to estimate SCD1 activity $(11,43,49)$. The validity of SCD1 $1_{(16)}$ as a marker of DNL has been tested against the isotope tracer deuterium oxide with some conflicting findings. A study by Lee et al. reported a strong positive association between SCD1(16) and DNL 
assessed through 10 days of $\mathrm{D}_{2} \mathrm{O}$ enrichment (48). In contrast, a more recent study by Rosqvist et al. suggests that there is no association between $\operatorname{SCD} 1_{(16)}$ and $\mathrm{DNL}\left(12 \mathrm{~h} \mathrm{D}_{2} \mathrm{O}\right.$ enrichment) when subjects consume their habitual diet (47). It is yet unclear what is at the basis of these discrepancies. Possibly, the differences in tracer enrichment duration play a role.

\subsection{Contribution to liver fat accumulation and influence of diet}

Both iv infusion of ${ }^{13} \mathrm{C}$-acetate tracer and oral intake of deuterated water have been used to assess the relative contribution of DNL to VLDL-TG, showing that DNL contributed to around $5-10 \%$ of VLDL-TG in healthy individuals in the fasted state, whereas contribution increased to around $20-25 \%$ in people with $\operatorname{NAFL}(12,15,16)$. In the postprandial state, the proportion of DNL derived fatty acids in VLDL-TG increased from 5-10\% in the fasted state up to $15-20 \%$ after a first meal and to $20-25 \%$ after a second meal in healthy individuals (16). In people with NAFL this increase seemed to be absent, with DNL contributions similar during fasting and postprandial periods (around $20 \%$ of TG-rich lipoproteins (tTRL-TG) after 3 days of ${ }^{13} \mathrm{C}$-acetate labeling) (15).

Effects of dietary interventions on DNL have also been studied frequently. Specifically, the effect of dietary carbohydrate and fat on DNL has been a topic of great interest. Using the before mentioned tracer methodologies, several studies indicate that high carbohydrate diets increase fasting and postprandial fractional DNL in both lean and obese subjects when compared to diets high in fat and similar in protein $(29,33,40,45,50,51)$ and that replacement of carbohydrates by both fat and protein leads to a reduction in fasting DNL (52). In addition, overfeeding with simple carbohydrates for 3-4 weeks has been shown to increase DNL, as measured by deuterated water and lipogenic index, parallel to an increase in liver fat $(43,44,53)$. The effect of carbohydrate intake on DNL may be dependent on the type of carbohydrate consumed, as DNL rates have been reported to be higher upon meals/diets high in fructose than meals/diets high in glucose or complex carbohydrates (23, $28,30)$. In line with these isotope tracer studies, carbohydrate type effects have also been shown using the lipogenic index. It has been shown in a small study population of three healthy volunteers that an increase in palmitate-rich and lineolate-poor VLDL-TG mediated by a 10-day high sugar diet can be reduced by 7-10 day substitution of dietary starch for sugar (54). Regarding the effect of fatty acids on DNL, Green et al. recently showed by using deuterated water that 8-week supplementation with the omega-3 fatty acids EPA and DHA at a dose of $4 \mathrm{~g} /$ day decreased both fasting and postprandial DNL compared to baseline in thirty-eight healthy men (13). 
Summarizing, DNL seems to be a significant contributor to liver fat, making it an interesting target for intervention studies aiming at decreasing liver fat storage. In this respect, diets high in carbohydrates stimulate DNL most, with fructose and simple sugars as most potent stimulators. Also, the lipogenic index has been shown to change upon dietary interventions stimulating DNL and therefore can render some valuable information on DNL in a less invasive and less costly way when studying dietary effects in large-scale studies.

\section{NEFA UPTAKE}

\subsection{Pathway description}

The largest contributor to hepatic fat originates from uptake of plasma non-esterified fatty acids (NEFA) $(15,16)$. The main source of plasma NEFAs is fatty acids originating from adipose tissue lipolysis, while spillover fatty acids can also contribute (15). These plasma NEFAs can be taken up by the liver through protein mediated transport (10). Uptake of NEFAs by the liver is strongly dependent on the level of NEFAs in the plasma, with lower plasma NEFA levels resulting in reduced uptake of NEFA by the liver (55).

\subsection{Techniques to measure this pathway}

\subsubsection{Stable isotope measurements in plasma}

Contribution of NEFA to liver fat can be assessed using intravenous infusion of palmitate tracer, to label the plasma NEFA pool, and subsequent determination of tracer enrichment in VLDL-TG. The assumption is made that palmitate is representative for all plasma free fatty acids with respect to turnover (56). Additionally, palmitate tracer is most commonly used as this fatty acid is highly abundant in plasma and is the cheapest fatty acid tracer. Basically, the method is similar to the method used to measure dietary fat uptake by labeling dietary fatty acids. However, by infusing the labeled palmitate instead of providing the tracer orally, the plasma NEFA pool is labeled and the palmitate that will be taken up by the liver will represent NEFA contribution to liver fat. Tracing back the labeled palmitate in VLDL-TG thus provides information on the contribution of plasma NEFA to liver fat. The most frequently used palmitate tracer is ${ }^{13} \mathrm{C}$-labeled palmitate $(12,15-17,29)$, but also intravenous deuterium palmitate tracers have been used to assess NEFA contribution to VLDL-TG (18, 57). The preferred tracer depends on the combination with other tracers (e.g. when for example ${ }^{13} \mathrm{C}$-palmitate is used to track dietary fat contribution, a deuterium labeled palmitate tracer is preferred to study hepatic NEFA uptake). 


\subsubsection{Radioisotope measurements in the liver}

Besides tracing the labeled fatty acids in VLDL-TG, fatty acid tracer has also been used in combination with PET imaging (58-60). In this respect, ${ }^{18} \mathrm{FTHA}$ tracer, the earlier mentioned long chain fatty acid analog containing ${ }^{18}$ fluorine, can be used to trace NEFA uptake by the liver. Upon intravenous injection, FTHA dilutes in the NEFA pool and can be taken up by the liver. As mentioned before, this tracer cannot be oxidized and therefore the amount of FTHA trapped in the liver determined with PET imaging provides information on the balance between hepatic NEFA uptake and export. Another fatty acid tracer that has been combined with PET imaging is ${ }^{11} \mathrm{C}$-labeled palmitate $(55,61)$. In contrast to $\mathrm{FTHA},{ }^{11} \mathrm{C}$-labeled palmitate can be oxidized completely. If this is taken into account by using compartmental modeling, also the uptake of FA can be determined.

\subsection{Contribution to liver fat accumulation and influence of diet}

The relative contribution of NEFA to VLDL-TG has been determined using ${ }^{13} \mathrm{C}$-palmitate in subjects with NAFL and healthy subjects. Under fasting conditions the proportion from NEFA in VLDL-TG was around $65 \%$ and $60 \%$ in healthy volunteers and volunteers with NAFL, respectively $(15,16)$, showing that NEFA uptake is the largest contributor to hepatic fat, at least in the fasted state. Of the NEFA pool approximately $80 \%$ was originating from adipose tissue in volunteers with NAFL in the fasting state, showing that adipose tissue is the main contributor to plasma NEFA. Postprandially, plasma insulin concentrations will rise, leading to suppression of adipose tissue lipolysis. In NAFL, adipose tissue contribution to NEFA decreases to around $60 \%$ in the fed state. The decrease in adipose tissue NEFA release into the circulation translates into a decreased uptake of plasma NEFA by the liver. NEFA contribution to VLDL-TG in the postprandial state decreased to around $40 \%$ in healthy participants (16). In participants with NAFL postprandial contribution was determined in tTRL-TG and decreased from $55 \%$ fasted to $32 \%$ postprandially (15). In addition, using PET methodology, an ${ }^{18}$ FTHA tracer study showed that fasting uptake of NEFA by the liver was impaired in people with impaired glucose tolerance (IGT) compared to healthy control subjects (59), whereas no differences in hepatic fat uptake have been shown between obese and lean subjects using infusion of ${ }^{11} \mathrm{C}$-palmitate in combination with PET (61).

Studies focusing on the impact of diet on hepatic NEFA contribution are limited. Comparing high carbohydrate/low fat diets to high fat/low carbohydrate diets, using either intravenous infusion of ${ }^{13} \mathrm{C}$-palmitate or ${ }^{2} \mathrm{H}_{2}$-palmitic acid tracer showed that fasting and 6-hour postmeal contributions of NEFA to VLDL-TG were similar in healthy volunteers $(11,29)$. Recently, NEFA contribution was compared between a 4-week relatively high-fat diet enriched in SFA 
and a 4-week relatively high-carbohydrate diet enriched in free sugars, using ${ }^{2} \mathrm{H}_{2}$-palmitate in sixteen overweight males (14). NEFA contribution was not increased upon high saturated fat intake when compared to high simple carbohydrate intake $6 \mathrm{~h}$ after meal consumption.

In summary, several studies have shown that NEFA uptake is the largest contributor to hepatic fat in the fasted state. Nevertheless, dietary studies are however limited and specifically the effects of different types of fat and carbohydrates on hepatic NEFA uptake using before mentioned tracer methodologies still awaits further study.

\section{FAT OXIDATION AND ACETYL-COA DISPOSAL}

\subsection{Pathway description}

So far, methods that focused on lipid storage were discussed. Here and in the next paragraph we discuss methods that focus on lipid disposal. Oxidation of hepatic fat is one of the pathways contributing to liver fat removal. Hepatic FA will be broken down to acetylCoA in mitochondrial $\beta$-oxidation. The formed acetyl-CoA can subsequently be used in the TCA cycle to be oxidized further, thereby producing $\mathrm{NADH}$, which serves as an electron donor needed in the oxidative phosphorylation to produce ATP. Alternatively, acetyl-CoA can be used for the formation of ketone bodies (62). Ketone body synthesis mainly takes place in the hepatic mitochondria via the formation of acetoacyl-CoA and $\beta$-hydroxy- $\beta$ methylglutaryl-CoA (HMG-CoA) and produces the ketone bodies acetoacetate, $\beta$ hydroxybutyrate $(\mathrm{BHB})$ and acetone. Ketogenesis takes place when acetyl-CoA formation exceeds its use by the TCA cycle, in such situation, acetyl-CoA accumulates and levels increase (63). As an example, ketone body formation is stimulated under fasted conditions, when fatty acid degradation to acetyl-CoA is increased (63).

\subsection{Techniques to measure this pathway}

\subsubsection{Direct measurements of hepatic fat oxidation}

A method that is used to measure complete oxidation of hepatic fatty acids is the combined use of intravenously infused ${ }^{11} \mathrm{C}$-palmitate tracer and PET imaging $(61,64)$. As mentioned before, by making use of modeling approaches several liver fat fluxes including hepatic palmitate oxidation can be determined (61). This is the only approach used to measure oxidation of hepatic fat directly. There are techniques that can determine hepatic TCA cycle flux using stable isotope tracers in combination with $\operatorname{MRS}(65,66)$ or plasma analyses $(67)$, and techniques determining complete hepatic oxidation by a ${ }^{13} \mathrm{C}$-methionine breath test 
(68-71), however such techniques do not specifically determine hepatic fat oxidation and therefore are outside the scope of this review.

\subsubsection{Indirect markers of hepatic fat oxidation and acetyl-CoA disposal}

Next to stable isotope measurements, indirect markers have been used to provide an indication of liver specific fat oxidation $(57,61,72,73)$. Most often, circulating betahydroxybutyrate (BHB) is used, which is widely accepted as marker for hepatic fat oxidation. Although plasma BHB levels are an indirect measure, it is seen as an organ-specific marker for liver fat oxidation as it is mainly produced by hepatic mitochondria. BHB is exported from the liver and can be used by other tissues. Therefore, circulating levels of BHB not only depend on the export rate from the liver, but also on extra-hepatic use. Nevertheless, lozzo and colleagues compared hepatic fat oxidation determined by the ${ }^{11} \mathrm{C}$-PET technique to circulating BHB levels and found a strong positive association between the two measurements (61).

\subsection{Contribution to liver fat accumulation and influence of diet}

To date, the importance of hepatic fat oxidation in relation to liver fat accumulation has only been studied by comparing BHB levels in people with and without NAFL and these studies show decreased $(74,75)$, increased $(76,77)$, and similar $(78)$ BHB levels in NAFL compared to people without NAFL. These discrepancies might be explained by differences in population characteristics, sample size and fasting time. Thus, these studies so far have not been able to provide a clear answer on the role of liver fat oxidation in the accumulation of liver fat. To answer this question the earlier mentioned PET methodology could be applied, providing direct instead of indirect information on liver fat oxidation. This methodology has already been used to compare liver fat oxidation in lean and obese subjects (61), and could in a similar way be used to compare people with and without NAFL.

Dietary effects have been studied extensively, mostly using blood BHB levels as a marker for hepatic fat oxidation. Plasma BHB levels increase upon consumption of a high fat meal, whereas BHB levels do not increase upon a carbohydrate rich meal (79). In line with this, a 3-day high fat/low carbohydrate diet and a 2-week high fat/low carbohydrate diet resulted in higher BHB levels compared to isoenergetic low fat/high carbohydrate diets $(57,72)$. Similar, upon a two-week low carbohydrate diet, higher in both fat and protein, BHB has been shown to increase compared to baseline (52). Furthermore, an 8-week high MUFAdiet has been shown to decrease fasting plasma BHB (when compared to baseline) in patients with type 2 diabetes (80). No changes in plasma BHB where found after an 8-week 
high carbohydrate/high fibre/high GI diet in patients with type 2 diabetes (80). However, no definitive conclusions regarding the effects of the type of fat and carbohydrate on liver fat oxidation can be drawn from these studies as effects were only compared to baseline.

Overall, direct hepatic fat oxidation methodology is not often applied, as this requires advanced stable isotope and imaging techniques and exposure to ionizing radiation. Such methodology would however be interesting to apply in studies investigating the role of liver fat oxidation in NAFL. In nutritional studies BHB has frequently been used as marker for hepatic fat oxidation, as it is a widely accepted and easy to determine blood marker. These nutritional studies have shown increased hepatic fat oxidation upon diets high in dietary fat and low in carbohydrates, but effects may differ depending on the type of carbohydrates or fatty acids. The latter has hardly been studied. While theoretically increased hepatic fat oxidation seems a good way to reduce liver fat accumulation, the physiological relevance of fat oxidation rates in determining hepatic fat is not well investigated. Future studies should measure hepatic fat oxidation directly in people with NAFL and in interventions combined with measurements of hepatic steatosis, in order to investigate whether decreased fat oxidation may underlie NAFL etiology.

\section{VLDL SECRETION}

\subsection{Pathway description}

Another pathway contributing to hepatic fat clearance is the secretion of VLDL. Liver TGs are used as component for VLDL assembly, together with cholesterol and apolipoproteins. Assembled TG rich VLDL particles can be secreted immediately or can be stored in the cytosol for delayed secretion (18). Upon secretion, liver TGs are transported within these VLDL particles through the circulation to peripheral tissues, where LPL ensures the release of the packed TGs.

\subsection{Techniques to measure this pathway}

\subsubsection{Tracers to determine VLDL particle production}

VLDL particle production has been assessed by studying the kinetics of apo B100, an important lipoprotein on VLDL particles. For its biosynthesis, amino acids are needed and therefore an amino acid tracer can be used to label apoB100 in order to study VLDL production. Different leucine tracers, such as trideuterated leucine and ${ }^{13} \mathrm{C}$-leucine, have been used to intravenously label apoB100 (81-85). The enrichment of leucine tracer in VLDL apoB100 can be determined with GC/MS and is used for the calculation of VLDL production 
based on compartment modeling $(81,83)$. However, changes in the amount of VLDL particles produced by the liver do not necessarily have to translate into changes in secreted VLDL-TG as the degree of TG loading also plays a role. For example, increased production of VLDL particles can take place without changes in VLDL-TG secretion, reflecting the formation of smaller particles (84).

\subsubsection{Tracers to determine VLDL-TG secretion}

To study the amount of TGs secreted from the liver in VLDL, ${ }^{13} \mathrm{C}$-glycerol and ${ }^{13} \mathrm{C}$-palmitate tracers can be used. Upon intravenous infusion, the labeled glycerol or palmitate will be incorporated into TG formed in the liver, which will subsequently be assembled in VLDL particles. Based on the incorporation curves of ${ }^{13} \mathrm{C}$-glycerol or ${ }^{13} \mathrm{C}$-palmitate in VLDL, the synthesis of VLDL-TG can then be determined $(18,29,84,86)$. Another palmitate tracer method that can provide information on the amount of TG released from the liver is ${ }^{11} \mathrm{C}$ palmitate PET imaging, as mentioned earlier (61).

In addition to glycerol and palmitate tracers, VLDL-TG tracers have been used $(64,72,87$, 88). The VLDL-TG tracer technique is based on the tracer dilution principle. Endogenous produced VLDL-TG by the liver, will dilute the infused, labeled VLDL-TG enrichment and therefore, absolute VLDL-TG secretion rates can be determined based on the tracer infusion rate and arterial tracer enrichment levels. The main advantage of this technique is that it is based on fewer assumptions than modeling based methods. VLDL-TG tracers however, have to be induced in vivo or ex vivo, as there are no VLDL-tracers commercially available.

\subsection{Contribution to liver fat accumulation and influence of diet}

To which extent VLDL secretion contributes to the development of hepatic steatosis is yet unclear, as studies investigating this are lacking. It is however known that people with mutations in ApoB genes develop NAFL, as is the case in familial hypobetalipoproteinemia (FHBL) (89). Liver fat content in FHBL individuals has been reported to be three times higher as compared to healthy individuals (89). Liver fat may accumulate in these people because of impaired VLDL assembly. The combined PET/ ${ }^{11} \mathrm{C}$ tracer technique has been used to show that of the liver fat disposal pathways, VLDL-TG secretion seems of less importance to hepatic fat disposal compared to fat oxidation (61). Furthermore, VLDL-TG secretion has been studied in different populations indicating similar TG secretion between lean and obese people (61), and increased TG secretion in people with hypertriglyceridemia (18) and type 2 diabetes (88) compared to healthy controls. In NAFL, VLDL-TG export is increased $(86,90)$ and therefore, changes in VLDL-TG export do not explain NAFL. Thus, the increased 
VLDL-TG export seems to be a consequence of NAFL rather than its cause. Nevertheless, changes in VLDL-TG export will always have consequences for liver fat storage.

Some dietary intervention studies have been performed in which VLDL-TG secretion was assessed. Conflicting results have been reported on the comparison of high carbohydrate/low fat and low carbohydrate/high fat diets on VLDL-TG secretion. In healthy lean subjects VLDL-TG secretion was increased upon a two-week high carbohydrate diet (75\% of energy as $\mathrm{CHO}$ vs $30 \%$ energy as $\mathrm{CHO}$ in $\mathrm{HF}$ diet) as determined with VLDL tracer (72), whereas Parks et al. did not find differences between a 5 week high carbohydrate/low fat diet and a 1 week control diet (consisting of $68 \%$ vs $50 \%$ energy as $\mathrm{CHO}$ ) in normolipidemic lean and hypertriglyceridemic overweight subjects when using ${ }^{13} \mathrm{C}$ palmitate tracer (29). Whether these conflicting results are due to the different study populations or the differences in carbohydrate and fat content between the 2 studies is unknown.

The effects of fish oils, linoleic acid and medium chain fatty acids (MCFA) have been studied on VLDL ApoB secretion. Three-week supplementation of $60 \mathrm{mg} / \mathrm{d}$ linoleic acid compared to MCFA did not result in differences in ApoB secretion (81). Six-week fish oil supplementation on the other hand showed reductions in VLDL ApoB secretion compared to placebo (corn oil) $(83,91)$. No differences in VLDL ApoB secretion have been shown upon 10-week ingestion of EPA, DHA or corn oil (85). In these studies VLDL-TG secretion was not determined.

Summarizing, little is known about the importance of TG secretion from the liver in determining liver fat accumulation. VLDL-TG export is increased in NAFL, indicating that changes in VLDL-TG are not causing NAFL. However, changes in VLDL-TG will have consequences for NAFL. A limited number of studies have investigated dietary effects on VLDL-TG secretion and similar to nutritional studies on hepatic fat oxidation, the physiological relevance of these nutritional findings remain unknown.

\section{CONCLUSIONS}

Hepatic fat accumulation is the result of an imbalance between lipid storage on the one hand and disposal on the other hand. The different pathways involved in fattening of the liver can accurately be measured by stable isotope techniques in combination with VLDLTG analysis or imaging techniques as MRS and by PET methodologies. As an alternative, a few validated plasma markers and indexes can be used as a more feasible alternative in studies with large sample sizes. Despite the availability of a wide range in techniques to measure these pathways, knowledge on the contribution of each pathway to liver fattening 
in humans in health and disease is still limited. This is most likely due to the specialized expertise and facilities needed to perform isotope tracer studies and the high costs of such studies. Furthermore, relative contributions depend on factors as study population and nutritional state (fed vs. fasted), factors which require more detailed investigation as well. While some specific questions with respect to dietary influence on pathway contributions have been answered, much remains unclear. Future research on the role of liver fat pathways in liver fat accumulation will improve our understanding of the mechanisms contributing to liver fat accumulation, which is crucial to the development of effective treatment strategies for NAFL and its associated metabolic disturbances. 


\section{ACKNOWLEDGEMENTS}

KR was in part financed by the Ministry of Economic Affairs and Climate Policy by means of the PPP Allowance made available by the Top Sector Life Sciences \& Health to stimulate public-private partnerships and by Unilever R\&D Wageningen. We acknowledge the support to KR and PS from the Netherlands Cardiovascular Research Initiative: an initiative with support of the Dutch Heart Foundation (CVON2014-02 ENERGISE). VS-H is a recipient of an ERC starting grant (grant no. 759161 'MRS in diabetes'). HP was an employee of Unilever at the time of this work and has since changed his professional affiliation to: Superfoods, Landsmeer. 


\section{REFERENCES}

1. Younossi Z, Anstee QM, Marietti M, Hardy T, Henry L, Eslam M, et al. Global burden of NAFLD and NASH: trends, predictions, risk factors and prevention. Nature reviews Gastroenterology \& hepatology. 2018;15(1):11-20.

2. Lim S, Oh TJ, Koh KK. Mechanistic link between nonalcoholic fatty liver disease and cardiometabolic disorders. International journal of cardiology. 2015;201:408-14.

3. Targher G, Day CP, Bonora E. Risk of cardiovascular disease in patients with nonalcoholic fatty liver disease. The New England journal of medicine. 2010;363(14):1341-50.

4. Targher G, Byrne CD. Clinical Review: Nonalcoholic fatty liver disease: a novel cardiometabolic risk factor for type 2 diabetes and its complications. The Journal of clinical endocrinology and metabolism. 2013;98(2):483-95.

5. Gaggini M, Morelli M, Buzzigoli E, DeFronzo RA, Bugianesi E, Gastaldelli A. Nonalcoholic fatty liver disease (NAFLD) and its connection with insulin resistance, dyslipidemia, atherosclerosis and coronary heart disease. Nutrients. 2013;5(5):1544-60.

6. Bellentani S, Saccoccio G, Masutti F, Croce LS, Brandi G, Sasso F, et al. Prevalence of and risk factors for hepatic steatosis in Northern Italy. Annals of internal medicine. 2000;132(2):112-7.

7. Iqbal J, Hussain MM. Intestinal lipid absorption. American journal of physiology Endocrinology and metabolism. 2009;296(6):E1183-94.

8. Cooper AD. Hepatic uptake of chylomicron remnants. Journal of lipid research. 1997;38(11):2173-92.

9. Hodson L, Frayn KN. Hepatic fatty acid partitioning. Current opinion in lipidology. 2011;22(3):216-24.

10. Musso G, Gambino R, Cassader M. Recent insights into hepatic lipid metabolism in non-alcoholic fatty liver disease (NAFLD). Progress in lipid research. 2009;48(1):126.

11. Chong MF, Hodson L, Bickerton AS, Roberts R, Neville M, Karpe F, et al. Parallel activation of de novo lipogenesis and stearoyl-CoA desaturase activity after $3 \mathrm{~d}$ of high-carbohydrate feeding. The American journal of clinical nutrition. 2008;87(4):817-23.

12. Lambert JE, Ramos-Roman MA, Browning JD, Parks EJ. Increased de novo lipogenesis is a distinct characteristic of individuals with nonalcoholic fatty liver disease. Gastroenterology. 2014;146(3):726-35.

13. Green CJ, Pramfalk C, Charlton CA, Gunn PJ, Cornfield T, Pavlides M, et al. Hepatic de novo lipogenesis is suppressed and fat oxidation is increased by omega-3 fatty acids at the expense of glucose metabolism. BMJ open diabetes research \& care. 2020;8(1).

14. Parry SA, Rosqvist F, Mozes FE, Cornfield T, Hutchinson M, Piche ME, et al. Intrahepatic Fat and Postprandial Glycemia Increase After Consumption of a Diet Enriched in Saturated Fat Compared With Free Sugars. Diabetes care. 2020. 
15. Donnelly KL, Smith Cl, Schwarzenberg SJ, Jessurun J, Boldt MD, Parks EJ. Sources of fatty acids stored in liver and secreted via lipoproteins in patients with nonalcoholic fatty liver disease. J Clin Invest. 2005;115(5):1343-51.

16. Timlin MT, Barrows BR, Parks EJ. Increased dietary substrate delivery alters hepatic fatty acid recycling in healthy men. Diabetes. 2005;54(9):2694-701.

17. Barrows BR, Parks EJ. Contributions of different fatty acid sources to very lowdensity lipoprotein-triacylglycerol in the fasted and fed states. J Clin Endocrinol Metab. 2006;91(4):1446-52.

18. Vedala A, Wang W, Neese RA, Christiansen MP, Hellerstein MK. Delayed secretory pathway contributions to VLDL-triglycerides from plasma NEFA, diet, and de novo lipogenesis in humans. Journal of lipid research. 2006;47(11):2562-74.

19. Peter A, Cegan A, Wagner S, Lehmann R, Stefan N, Königsrainer A, et al. Hepatic lipid composition and stearoyl-coenzyme A desaturase 1 mRNA expression can be estimated from plasma VLDL fatty acid ratios. Clinical chemistry. 2009;55(12):211320.

20. Ravikumar B, Carey PE, Snaar JE, Deelchand DK, Cook DB, Neely RD, et al. Real-time assessment of postprandial fat storage in liver and skeletal muscle in health and type 2 diabetes. American journal of physiology Endocrinology and metabolism. 2005;288(4):E789-97.

21. Lindeboom L, de Graaf RA, Nabuurs Cl, van Ewijk PA, Hesselink MK, Wildberger JE, et al. Quantum coherence spectroscopy to measure dietary fat retention in the liver. JCl insight. 2016;1(13):e84671.

22. Labbe SM, Grenier-Larouche T, Noll C, Phoenix S, Guerin B, Turcotte EE, et al. Increased myocardial uptake of dietary fatty acids linked to cardiac dysfunction in glucose-intolerant humans. Diabetes. 2012;61(11):2701-10.

23. Low WS, Cornfield T, Charlton CA, Tomlinson JW, Hodson L. Sex Differences in Hepatic De Novo Lipogenesis with Acute Fructose Feeding. Nutrients. 2018;10(9).

24. Ferramosca A, Zara V. Modulation of hepatic steatosis by dietary fatty acids. World journal of gastroenterology. 2014;20(7):1746-55.

25. Hellerstein MK, Schwarz JM, Neese RA. Regulation of hepatic de novo lipogenesis in humans. Annual review of nutrition. 1996;16:523-57.

26. Hellerstein MK, Neese RA. Mass isotopomer distribution analysis: a technique for measuring biosynthesis and turnover of polymers. The American journal of physiology. 1992;263(5 Pt 1):E988-1001.

27. Marques-Lopes I, Ansorena D, Astiasaran I, Forga L, Martinez JA. Postprandial de novo lipogenesis and metabolic changes induced by a high-carbohydrate, low-fat meal in lean and overweight men. The American journal of clinical nutrition. 2001;73(2):253-61.

28. Stanhope KL, Schwarz JM, Keim NL, Griffen SC, Bremer AA, Graham JL, et al. Consuming fructose-sweetened, not glucose-sweetened, beverages increases visceral adiposity and lipids and decreases insulin sensitivity in overweight/obese humans. The Journal of clinical investigation. 2009;119(5):1322-34. 
29. Parks EJ, Krauss RM, Christiansen MP, Neese RA, Hellerstein MK. Effects of a lowfat, high-carbohydrate diet on VLDL-triglyceride assembly, production, and clearance. The Journal of clinical investigation. 1999;104(8):1087-96.

30. Schwarz JM, Noworolski SM, Wen MJ, Dyachenko A, Prior JL, Weinberg ME, et al. Effect of a High-Fructose Weight-Maintaining Diet on Lipogenesis and Liver Fat. The Journal of clinical endocrinology and metabolism. 2015;100(6):2434-42.

31. Hellerstein MK, Neese RA, Schwarz JM. Model for measuring absolute rates of hepatic de novo lipogenesis and reesterification of free fatty acids. The American journal of physiology. 1993;265(5 Pt 1):E814-20.

32. Aarsland A, Chinkes D, Wolfe RR. Contributions of de novo synthesis of fatty acids to total VLDL-triglyceride secretion during prolonged hyperglycemia/hyperinsulinemia in normal man. The Journal of clinical investigation. 1996;98(9):2008-17.

33. Hudgins LC, Hellerstein M, Seidman C, Neese R, Diakun J, Hirsch J. Human fatty acid synthesis is stimulated by a eucaloric low fat, high carbohydrate diet. The Journal of clinical investigation. 1996;97(9):2081-91.

34. Stiede K, Miao W, Blanchette HS, Beysen C, Harriman G, Harwood HJ, Jr., et al. Acetyl-coenzyme A carboxylase inhibition reduces de novo lipogenesis in overweight male subjects: A randomized, double-blind, crossover study. Hepatology (Baltimore, Md). 2017;66(2):324-34.

35. C. Beysen TEA, M.K. Hellerstein, S.M. Turner. Isotopic Tracers for the Measurement of Metabolic Flux Rates. In: A.J. Krentz CW, M. Hompesch, editor. Translational Research Methods in Diabetes, Obesity, and Nonalcoholic Fatty Liver Disease2019.

36. Matikainen N, Adiels M, Soderlund S, Stennabb S, Ahola T, Hakkarainen A, et al. Hepatic lipogenesis and a marker of hepatic lipid oxidation, predict postprandial responses of triglyceride-rich lipoproteins. Obesity (Silver Spring). 2014;22(8):1854-9.

37. Mancina RM, Matikainen N, Maglio C, Soderlund S, Lundbom N, Hakkarainen A, et al. Paradoxical dissociation between hepatic fat content and de novo lipogenesis due to PNPLA3 sequence variant. J Clin Endocrinol Metab. 2015;100(5):E821-5.

38. Petersen KF, Dufour S, Savage DB, Bilz S, Solomon G, Yonemitsu S, et al. The role of skeletal muscle insulin resistance in the pathogenesis of the metabolic syndrome. Proceedings of the National Academy of Sciences of the United States of America. 2007;104(31):12587-94.

39. McDevitt RM, Bott SJ, Harding M, Coward WA, Bluck LJ, Prentice AM. De novo lipogenesis during controlled overfeeding with sucrose or glucose in lean and obese women. The American journal of clinical nutrition. 2001;74(6):737-46.

40. Wilke MS, French MA, Goh YK, Ryan EA, Jones PJ, Clandinin MT. Synthesis of specific fatty acids contributes to VLDL-triacylglycerol composition in humans with and without type 2 diabetes. Diabetologia. 2009;52(8):1628-37.

41. Diraison F, Moulin P, Beylot M. Contribution of hepatic de novo lipogenesis and reesterification of plasma non esterified fatty acids to plasma triglyceride synthesis during non-alcoholic fatty liver disease. Diabetes Metab. 2003;29(5):478-85. 
42. Paglialunga S, Dehn CA. Clinical assessment of hepatic de novo lipogenesis in nonalcoholic fatty liver disease. Lipids in health and disease. 2016;15(1):159.

43. Silbernagel G, Kovarova M, Cegan A, Machann J, Schick F, Lehmann R, et al. High hepatic SCD1 activity is associated with low liver fat content in healthy subjects under a lipogenic diet. The Journal of clinical endocrinology and metabolism. 2012;97(12):E2288-92.

44. Sevastianova K, Santos A, Kotronen A, Hakkarainen A, Makkonen J, Silander K, et al. Effect of short-term carbohydrate overfeeding and long-term weight loss on liver fat in overweight humans. The American journal of clinical nutrition. 2012;96(4):727-34.

45. Hudgins LC, Hellerstein MK, Seidman CE, Neese RA, Tremaroli JD, Hirsch J. Relationship between carbohydrate-induced hypertriglyceridemia and fatty acid synthesis in lean and obese subjects. Journal of lipid research. 2000;41(4):595-604.

46. Hudgins LC, Parker TS, Levine DM, Hellerstein MK. A dual sugar challenge test for lipogenic sensitivity to dietary fructose. J Clin Endocrinol Metab. 2011;96(3):861-8.

47. Rosqvist F, McNeil CA, Pramfalk C, Parry SA, Low WS, Cornfield T, et al. Fasting hepatic de novo lipogenesis is not reliably assessed using circulating fatty acid markers. The American journal of clinical nutrition. 2019;109(2):260-8.

48. Lee JJ, Lambert JE, Hovhannisyan Y, Ramos-Roman MA, Trombold JR, Wagner DA, et al. Palmitoleic acid is elevated in fatty liver disease and reflects hepatic lipogenesis. The American journal of clinical nutrition. 2015;101(1):34-43.

49. Fabbrini E, Magkos F, Su X, Abumrad NA, Nejedly N, Coughlin CC, et al. Insulin sensitivity is not associated with palmitoleate availability in obese humans. Journal of lipid research. 2011;52(4):808-12.

50. Schwarz JM, Neese RA, Turner S, Dare D, Hellerstein MK. Short-term alterations in carbohydrate energy intake in humans. Striking effects on hepatic glucose production, de novo lipogenesis, lipolysis, and whole-body fuel selection. The Journal of clinical investigation. 1995;96(6):2735-43.

51. Schwarz JM, Linfoot P, Dare D, Aghajanian K. Hepatic de novo lipogenesis in normoinsulinemic and hyperinsulinemic subjects consuming high-fat, lowcarbohydrate and low-fat, high-carbohydrate isoenergetic diets. The American journal of clinical nutrition. 2003;77(1):43-50.

52. Mardinoglu A, Wu H, Bjornson E, Zhang C, Hakkarainen A, Rasanen SM, et al. An Integrated Understanding of the Rapid Metabolic Benefits of a CarbohydrateRestricted Diet on Hepatic Steatosis in Humans. Cell metabolism. 2018;27(3):55971 e5.

53. Luukkonen PK, Sädevirta S, Zhou Y, Kayser B, Ali A, Ahonen L, et al. Saturated Fat Is More Metabolically Harmful for the Human Liver Than Unsaturated Fat or Simple Sugars. Diabetes care. 2018;41(8):1732-9.

54. Hudgins LC, Seidman CE, Diakun J, Hirsch J. Human fatty acid synthesis is reduced after the substitution of dietary starch for sugar. The American journal of clinical nutrition. 1998;67(4):631-9.

55. Rigazio S, Lehto HR, Tuunanen H, Nagren K, Kankaanpaa M, Simi C, et al. The lowering of hepatic fatty acid uptake improves liver function and insulin sensitivity 
without affecting hepatic fat content in humans. American journal of physiology Endocrinology and metabolism. 2008;295(2):E413-9.

56. Magkos F, Mittendorfer B. Stable isotope-labeled tracers for the investigation of fatty acid and triglyceride metabolism in humans in vivo. Clinical lipidology. 2009;4(2):215-30.

57. Roberts R, Bickerton AS, Fielding BA, Blaak EE, Wagenmakers AJ, Chong MF, et al. Reduced oxidation of dietary fat after a short term high-carbohydrate diet. The American journal of clinical nutrition. 2008;87(4):824-31.

58. Iozzo P, Turpeinen AK, Takala T, Oikonen V, Solin O, Ferrannini E, et al. Liver uptake of free fatty acids in vivo in humans as determined with $14(\mathrm{R}, \mathrm{S})$-[18F]fluoro-6thia-heptadecanoic acid and PET. European journal of nuclear medicine and molecular imaging. 2003;30(8):1160-4.

59. Iozzo P, Turpeinen AK, Takala T, Oikonen V, Bergman J, Gronroos T, et al. Defective liver disposal of free fatty acids in patients with impaired glucose tolerance. The Journal of clinical endocrinology and metabolism. 2004;89(7):3496-502.

60. Immonen H, Hannukainen JC, Kudomi N, Pihlajamaki J, Saunavaara V, Laine J, et al. Increased Liver Fatty Acid Uptake Is Partly Reversed and Liver Fat Content Normalized After Bariatric Surgery. Diabetes care. 2018;41(2):368-71.

61. Iozzo P, Bucci M, Roivainen A, Nagren K, Jarvisalo MJ, Kiss J, et al. Fatty acid metabolism in the liver, measured by positron emission tomography, is increased in obese individuals. Gastroenterology. 2010;139(3):846-56, 56 e1-6.

62. McGarry JD, Foster DW. Regulation of hepatic fatty acid oxidation and ketone body production. Annual review of biochemistry. 1980;49:395-420.

63. Fabbrini E, Magkos F. Hepatic Steatosis as a Marker of Metabolic Dysfunction. Nutrients. 2015;7(6):4995-5019.

64. Gormsen LC, Sondergaard E, Christensen NL, Jakobsen S, Nielsen EHT, Munk OL, et al. Metformin does not affect postabsorptive hepatic free fatty acid uptake, oxidation or resecretion in humans: A 3-month placebo-controlled clinical trial in patients with type 2 diabetes and healthy controls. Diabetes, obesity \& metabolism. 2018;20(6):1435-44.

65. Befroy DE, Perry RJ, Jain N, Dufour S, Cline GW, Trimmer JK, et al. Direct assessment of hepatic mitochondrial oxidative and anaplerotic fluxes in humans using dynamic 13C magnetic resonance spectroscopy. Nat Med. 2014;20(1):98-102.

66. Petersen KF, Befroy DE, Dufour S, Rothman DL, Shulman GI. Assessment of Hepatic Mitochondrial Oxidation and Pyruvate Cycling in NAFLD by (13)C Magnetic Resonance Spectroscopy. Cell Metab. 2016;24(1):167-71.

67. Sunny NE, Parks EJ, Browning JD, Burgess SC. Excessive hepatic mitochondrial TCA cycle and gluconeogenesis in humans with nonalcoholic fatty liver disease. Cell metabolism. 2011;14(6):804-10.

68. Banasch M, Ellrichmann M, Tannapfel A, Schmidt WE, Goetze O. The non-invasive (13)C-methionine breath test detects hepatic mitochondrial dysfunction as a marker of disease activity in non-alcoholic steatohepatitis. European journal of medical research. 2011;16(6):258-64. 
69. Armuzzi A, Marcoccia S, Zocco MA, De Lorenzo A, Grieco A, Tondi P, et al. NonInvasive assessment of human hepatic mitochondrial function through the 13Cmethionine breath test. Scandinavian journal of gastroenterology. 2000;35(6):6503.

70. Duro D, Duggan C, Valim C, Bechard L, Fitzgibbons S, Jaksic T, et al. Novel intravenous (13)C-methionine breath test as a measure of liver function in children with short bowel syndrome. Journal of pediatric surgery. 2009;44(1):236-40; discussion 40.

71. Milazzo L, Piazza M, Sangaletti O, Gatti N, Cappelletti A, Adorni F, et al. [13C]Methionine breath test: a novel method to detect antiretroviral drug-related mitochondrial toxicity. The Journal of antimicrobial chemotherapy. 2005;55(1):849.

72. Mittendorfer B, Sidossis LS. Mechanism for the increase in plasma triacylglycerol concentrations after consumption of short-term, high-carbohydrate diets. The American journal of clinical nutrition. 2001;73(5):892-9.

73. Browning JD, Baker JA, Rogers T, Davis J, Satapati S, Burgess SC. Short-term weight loss and hepatic triglyceride reduction: evidence of a metabolic advantage with dietary carbohydrate restriction. The American journal of clinical nutrition. 2011;93(5):1048-52.

74. Fletcher JA, Deja S, Satapati S, Fu X, Burgess SC, Browning JD. Impaired ketogenesis and increased acetyl-CoA oxidation promote hyperglycemia in human fatty liver. $\mathrm{JCl}$ insight. 2019;5(11).

75. Croci I, Byrne NM, Choquette S, Hills AP, Chachay VS, Clouston AD, et al. Wholebody substrate metabolism is associated with disease severity in patients with nonalcoholic fatty liver disease. Gut. 2013;62(11):1625-33.

76. Bugianesi E, Gastaldelli A, Vanni E, Gambino R, Cassader M, Baldi S, et al. Insulin resistance in non-diabetic patients with non-alcoholic fatty liver disease: sites and mechanisms. Diabetologia. 2005;48(4):634-42.

77. Männistö VT, Simonen M, Hyysalo J, Soininen P, Kangas AJ, Kaminska D, et al. Ketone body production is differentially altered in steatosis and non-alcoholic steatohepatitis in obese humans. Liver international : official journal of the International Association for the Study of the Liver. 2015;35(7):1853-61.

78. Kotronen A, Seppälä-Lindroos A, Vehkavaara S, Bergholm R, Frayn KN, Fielding BA, et al. Liver fat and lipid oxidation in humans. Liver international : official journal of the International Association for the Study of the Liver. 2009;29(9):1439-46.

79. Surina DM, Langhans W, Pauli R, Wenk C. Meal composition affects postprandial fatty acid oxidation. The American journal of physiology. 1993;264(6 Pt 2):R106570.

80. Bozzetto L, Costabile G, Luongo D, Naviglio D, Cicala V, Piantadosi C, et al. Reduction in liver fat by dietary MUFA in type 2 diabetes is helped by enhanced hepatic fat oxidation. Diabetologia. 2016;59(12):2697-701.

81. van Schalkwijk DB, Pasman WJ, Hendriks HF, Verheij ER, Rubingh CM, van Bochove $\mathrm{K}$, et al. Dietary medium chain fatty acid supplementation leads to reduced VLDL 
lipolysis and uptake rates in comparison to linoleic acid supplementation. PloS one. 2014;9(7):e100376.

82. Watts GF, Riches FM, Kelly JM, Powell MA, Croft KD. Determinants of the kinetics of very low-density lipoprotein apolipoprotein B-100 in non-obese men. Clinical and experimental pharmacology \& physiology. 1997;24(8):556-62.

83. Chan DC, Watts GF, Mori TA, Barrett PH, Redgrave TG, Beilin L. Randomized controlled trial of the effect of n-3 fatty acid supplementation on the metabolism of apolipoprotein B-100 and chylomicron remnants in men with visceral obesity. The American journal of clinical nutrition. 2003;77(2):300-7.

84. Smith GI, Magkos F, Reeds DN, Okunade AL, Patterson BW, Mittendorfer B. One day of mixed meal overfeeding reduces hepatic insulin sensitivity and increases VLDL particle but not VLDL-triglyceride secretion in overweight and obese men. The Journal of clinical endocrinology and metabolism. 2013;98(8):3454-62.

85. Allaire J, Vors C, Tremblay AJ, Marin J, Charest A, Tchernof A, et al. High-Dose DHA Has More Profound Effects on LDL-Related Features Than High-Dose EPA: The ComparED Study. The Journal of clinical endocrinology and metabolism. 2018;103(8):2909-17.

86. Fabbrini E, Mohammed BS, Magkos F, Korenblat KM, Patterson BW, Klein S. Alterations in adipose tissue and hepatic lipid kinetics in obese men and women with nonalcoholic fatty liver disease. Gastroenterology. 2008;134(2):424-31.

87. Gormsen LC, Jensen MD, Nielsen S. Measuring VLDL-triglyceride turnover in humans using ex vivo-prepared VLDL tracer. Journal of lipid research. 2006;47(1):99-106.

88. Sorensen LP, Andersen IR, Sondergaard E, Gormsen LC, Schmitz O, Christiansen JS, et al. Basal and insulin mediated VLDL-triglyceride kinetics in type 2 diabetic men. Diabetes. 2011;60(1):88-96.

89. Tanoli $T$, Yue $P$, Yablonskiy D, Schonfeld $G$. Fatty liver in familial hypobetalipoproteinemia: roles of the APOB defects, intra-abdominal adipose tissue, and insulin sensitivity. Journal of lipid research. 2004;45(5):941-7.

90. Toledo FG, Sniderman AD, Kelley DE. Influence of hepatic steatosis (fatty liver) on severity and composition of dyslipidemia in type 2 diabetes. Diabetes care. 2006;29(8):1845-50.

91. Chan DC, Watts GF, Barrett PH, Beilin LJ, Redgrave TG, Mori TA. Regulatory effects of HMG CoA reductase inhibitor and fish oils on apolipoprotein B-100 kinetics in insulin-resistant obese male subjects with dyslipidemia. Diabetes. 2002;51(8):2377-86. 
Liver fat storage and disposal pathways 


\section{CHAPTER 3}

\section{Hepatic saturated fatty acid fraction is associated with de novo lipogenesis and hepatic insulin resistance}

Kay H.M. Roumans ${ }^{1,{ }^{*}}$, Lucas Lindeboom ${ }^{1,2,{ }^{*}}$, Pandichelvam Veeraiah ${ }^{1,2}$,

Carlijn M.E. Remie ${ }^{1}$, Esther Phielix ${ }^{1}$, Bas Havekes ${ }^{3}$, Yvonne M.H. Bruls ${ }^{1,2}$,

Martijn C.G.J. Brouwers ${ }^{3}$, Marcus Ståhlman ${ }^{4}$, Marjan Alssema ${ }^{5}$, Harry P.F.

Peters $^{5}$, Renée de Mutsert ${ }^{6}$, Bart Staels ${ }^{7}$, Marja-Riitta Taskinen ${ }^{8}$, Jan

Borén ${ }^{4}$, Patrick Schrauwen ${ }^{1}$, Vera B. Schrauwen-Hinderling ${ }^{1,2}$

${ }^{1}$ Department of Nutrition and Movement Sciences, Maastricht University, P.O. BOX 616, 6200 MD, Maastricht, The Netherlands.

${ }^{2}$ Department of Radiology and Nuclear Medicine, Maastricht University Medical Center, P.O. BOX 5800, 6202 AZ, Maastricht, The Netherlands.

${ }^{3}$ Department of Internal Medicine, Division of Endocrinology and Metabolic Disease, Maastricht University Medical Center, P.O. BOX 5800, 6202 AZ, Maastricht, The Netherlands.

${ }^{4}$ Department of Molecular and Clinical Medicine, University of Gothenburg, and Sahlgrenska University Hospital, P.O. Box 428, 40530, Gothenburg, Sweden.

${ }^{5}$ Unilever Food Innovation Center, Plantage 14, 6708 WJ Wageningen, The Netherlands. ${ }^{6}$ Department of Clinical Epidemiology, Leiden University Medical Center, P.O. box 9600, 2300 RC, Leiden, The Netherlands.

${ }^{7}$ Univ. Lille, Inserm, CHU Lille, Institut Pasteur de Lille, U1011 - EGID, F-59000 Lille, France ${ }^{8}$ Research Program, Unit Clinical and Molecular Metabolism, University of Helsinki, P.O box 63 (Haartmaninkatu 8), 00014 Helsinki, Finland.

${ }^{*}$ Authors contributed equally.

Nat Commun. 2020 Apr 20;11(1):1891. 


\section{ABSTRACT}

Hepatic steatosis is associated with poor cardiometabolic health, with de novo lipogenesis (DNL) contributing to hepatic steatosis and subsequent insulin resistance. Hepatic saturated fatty acids (SFA) may be a marker of DNL and are suggested to be most detrimental in contributing to insulin resistance. Here we show in a cross-sectional study design (ClinicalTrials.gov ID: NCT03211299) that we are able to distinguish the fractions of hepatic SFA, mono- and polyunsaturated fatty acids in healthy and metabolically compromised volunteers using proton magnetic resonance spectroscopy $\left({ }^{1} \mathrm{H}-\mathrm{MRS}\right)$. DNL is positively associated with SFA fraction and is elevated in patients with non-alcoholic fatty liver and type 2 diabetes. Intriguingly, SFA fraction shows a strong, negative correlation with hepatic insulin sensitivity. Our results show that the hepatic lipid composition, as determined by our ${ }^{1} \mathrm{H}-\mathrm{MRS}$ methodology, is a measure of DNL and suggest that specifically the SFA fraction may hamper hepatic insulin sensitivity. 


\section{INTRODUCTION}

Non-alcoholic fatty liver (NAFL) is the most common cause of chronic liver disease, with estimated prevalence rates of $20-35 \%$ in Western countries (1). In obese people NAFL prevalence rates as high as $50-70 \%$ have been reported (2). NAFL can progress to steatohepatitis, fibrosis, and cirrhosis, which can lead to liver failure, and hepatocellular carcinoma (3). Moreover, ectopic fat accumulation in the liver is associated with impairments in cardiometabolic health $(4,5)$. In this respect, NAFL has been shown to be associated with insulin resistance on hepatic and whole-body level (6-9).

However, not all individuals with NAFL will develop insulin resistance, steatohepatitis or other liver disease, and it is of utmost clinical importance to understand which factors contribute to a pathologic fatty liver. It has been suggested that the pathway by which fat accumulation in the liver occurs may impact the clinical outcome. It has been shown that high rates of de novo lipogenesis (DNL) are associated with metabolic risk (10-12). In addition, animal experiments have suggested that the degree of saturation of the accumulating fatty acids in the liver may impact the metabolic consequences, with more saturated fatty acids (SFA) leading to worsened metabolic outcome (13). Interestingly, the end product of de novo synthesis of fatty acids is mainly SFA and therefore, high rates of DNL may result in a higher proportion of hepatic SFA, possibly explaining why DNL is associated with poor metabolic health. These findings stress the importance for a more detailed characterisation of hepatic lipid composition in humans, to ultimately understand the risk factors for the development of hepatic insulin resistance and disease.

However, in humans such data is very scarce, mainly due to the invasiveness of liver biopsy procedures that are needed. Therefore, only very few studies have determined hepatic fat composition, and these studies have been performed in patients in which liver biopsies were justified due to their risk for liver disease. Generally, these studies showed higher mono-unsaturated fatty acids (MUFA) fraction at the expense of the poly-unsaturated fatty acids (PUFA) fraction in people with NAFL compared to people without NAFL $(14,15)$. Earlier MR-based studies already investigated some parameters that are linked to degree of unsaturation(16-19), however these do not specifically and robustly quantify hepatic SFA, MUFA and PUFA fraction separately.

Here, we develop, validate and apply a magnetic resonance (MR) post-processing tool that enables to non-invasively quantify the fractions of hepatic SFA, MUFA and PUFA separately, in healthy and metabolically compromised human volunteers. Using this methodology, we test the hypothesis that higher rates of DNL are associated with an increased fraction of SFA in human liver. We also investigate if populations at higher risk to develop metabolic 
complications are characterized by altered hepatic fatty acid composition, and whether hepatic fatty acid composition is related to hepatic insulin sensitivity. We show that our ${ }^{1} \mathrm{H}$ MRS postprocessing methodology can be used to measure hepatic fatty acid composition in humans, that hepatic SFA content is strongly related to rates of DNL and that specifically the hepatic SFA fraction is related to hepatic insulin resistance.

\section{METHODS}

\section{Clinical Study Design}

The study was conducted at Maastricht University Medical Center, the Netherlands, between August 2017 and June 2018, and was approved by the Medical Ethical Committee of Maastricht University Medical Centre. Research was performed in accordance with relevant ethical regulations regarding human research participants. The study was registered at clinicaltrials.gov with identifier NCT03211299.

\section{Participants}

All participants recruited for this study provided written informed consent. Twenty-two healthy overweight/obese participants (BMI $27-35 \mathrm{~kg} / \mathrm{m}^{2}$ ), aged $45-70$ years with a large range in liver fat content (0.9-38.4\%) were recruited for this study. Female study participants were postmenopausal. Exclusion criteria were engagement in exercise for more than 2 hours per week, unstable body weight (weight loss or gain more than $3 \mathrm{~kg}$ in 3 months preceding enrollment), alcohol consumption more than 2 units per day, smoking more than 5 cigarettes per day, contra-indication for MRI, use of anti-coagulants, use of other medication known to interfere with the outcome parameters, diabetes or other active disease. Participants participated in an MRS measurement, deuterated water measurement and two-step hyperinsulinemic-euglycemic clamp, including baseline subcutaneous adipose tissue biopsy. All measurements took place within a time window of six weeks. Two days before each of the measurements, participants were instructed to refrain from physical exercise and alcohol consumption. The evening before the measurements, participants consumed a standardised high carbohydrate diner and fasted overnight. For group comparisons, two patient groups were included in the study: nine patients with type 2 diabetes and seven patients with glycogen storage disease type 1a. Patients with type 2 diabetes were aged 40-75 years, had a BMI between $25-38 \mathrm{~kg} / \mathrm{m}^{2}$ and relatively well controlled type 2 diabetes: HbA1c $<9.5 \%$. Patients had stable dietary habits, were on treatment with oral medication only (Metformin, Tolbutamide or Gliclazide) and did not use other medication known to interfere with the outcome parameters. Female patients were 
postmenopausal. Exclusion criteria were engagement in exercise for more than 3 hours per week, uncontrolled hypertension, anemia, unstable body weight (weight loss or gain more than $5 \mathrm{~kg}$ in 3 months preceding enrollment), alcohol or drug abuse, being vegetarian or vegan, having significant food allergies, contra-indication for MRI, use of anti-coagulants and clinically relevant active disease. GSD1a patients were recruited aged 18 years and older, clinically diagnosed with GSD1a and without contra-indications for MRI. In vivo validation in adipose tissue was performed in a subgroup of the healthy overweight/obese participants $(n=8)$. Reproducibility measurements in the liver were performed in a separate group of individuals aged between 25-71 years and BMI between $25.7-38.5 \mathrm{~kg} / \mathrm{m}^{2}(\mathrm{n}=7)$.

\section{Overview of specified outcomes}

The primary outcome was lipid composition (SFA fraction) as measured by Magnetic Resonance Spectroscopy and rates of de novo lipogenesis as measured by incorporation of deuterated water. Secondary outcome was hepatic insulin sensitivity as determined by suppression of hepatic glucose output during the low insulin phase in a 2-step hyperinsulinemic euglycemic clamp.

\section{Measurement of lipid content and lipid composition}

In this study, the lipid content and lipid composition (fraction of hepatic SFA, MUFA and PUFA) were determined by proton magnetic resonance spectroscopy ( ${ }^{1} \mathrm{H}-\mathrm{MRS}$ ) (healthy overweight/obese controls without NAFL: $n=7$ for total liver fat content, $n=6$ for liver fat composition, healthy overweight/obese with NAFL: $n=15$, patients with type 2 diabetes: $n=9$ and patients with GSD type $1 \mathrm{a}: \mathrm{n}=7$ ). All ${ }^{1} \mathrm{H}-\mathrm{MRS}$ experiments were performed on a $3 \mathrm{~T} \mathrm{MR}$ system (Achieva 3T-X Philips Healthcare, Best, Netherlands) by using a 32-channel sense cardiac/torso coil (Philips Healthcare, Best, Netherlands). All spectra were obtained by using a STEAM sequence (20) with the following parameters; repetition time (TR) $4500 \mathrm{~ms} /$ echo time (TE) $20 \mathrm{~ms} /$ mixing time (TM) 16 ms, spectral bandwidth $2000 \mathrm{~Hz}$ and data points 2048 . For the in vivo hepatic lipid spectra VAPOR water suppression (21) was applied and an additional water reference scan was obtained. The number of averages was 16 for the phantom experiments and in adipose tissue and 128 for the in vivo hepatic spectra. We used a voxel size of $30 \times 30 \times 30 \mathrm{~mm}$ for the hepatic and $15 \times 15 \times 15 \mathrm{~mm}$ for the adipose tissue measurements.

All obtained lipid spectra were post-processed in a home-written MATLAB (MATLAB 2014b, The MathWorks, Inc., Natick, Massachusetts, United States.) script in which, prior to fitting, all spectra are individually corrected for phase and frequency shift. Additionally, we 
performed eddy current correction for the individual lipid spectra. Phasing, frequency alignment and eddy current correction were all performed on individual spectra before signal averaging. Bad quality spectra from in vivo (e.g. due to motion) were removed automatically. For this, the linewidth, amplitude and frequency offset of the peaks in each individual spectrum were compared to the average values of all spectra.

For the setup of the post-processing routine we used a four-step approach. First, we acquired both ${ }^{1} \mathrm{H}$ and ${ }^{13} \mathrm{C}$ high-resolution (HR) NMR spectra from five different oils (olive, arachis, sunflower, safflower and rice oil), by using a pulse-acquire sequence. The lipid composition of the different oils was determined by integration of the peaks in the methyl region in the ${ }^{13} \mathrm{C}$ HR spectra. In the ${ }^{1} \mathrm{H}$ HR spectra four different regions were integrated, corresponding to the methyl protons (around $0.90 \mathrm{ppm}$ ), the allylic protons (around 2.02 $\mathrm{ppm}$ ), the alpha carbonyl group (around $2.20 \mathrm{ppm}$ ) and the diallylic protons (around 2.75 ppm) (supplementary figure 1). The ${ }^{1} \mathrm{H}$ HR spectra were used to develop a basis set used by the developed fitting algorithm. The lipid signal was described by 16 individual resonances ( 2 for the methyl group, 5 for the methylene group, 1 for the beta methyle-group, 2 for the allylic group, 2 for the alpha-carbonyl group and 1 for the diallylic group). In this basis set, the relative frequency shifts, the splitting patterns and the initial linewidths were described.

In a second setup step, we acquired spectra from the different oils on our clinical 3T scanner. Eddy current correction was applied based on an additional water reference scan with an identical experimental setup. The oil spectra were fitted with the developed Matlab algorithm. To this end, using the basis set from the HR spectra, the time domain signal was simulated. The amplitudes and relative frequency shifts of the individual peaks were updated iteratively. In each step the difference between the simulated spectrum and the acquired spectrum was minimized in the frequency domain. Next to these individual parameters, also the Gaussian line broadening, the Lorentzian line broadening, the zero order phase, an overall frequency shift and the baseline offset were automatically updated, affecting all the peaks in an identical fashion. Essential for our approach, we furthermore fixed the ratio of the methyl group and the alpha-carbonyl group (which overlaps with the allylic group at $3 \mathrm{~T}$ ) to 0.6 . Theoretically this factor should be 0.67 , as the methyl group contains 3 protons and the alpha-carbonyl group contains 2 protons and both groups are present only once in every fatty acid. However, due to TE-induced losses this factor will be affected. Therefore, this factor was determined empirically. In a final fine-tuning step of the fitting routine, the zero-order phase was accurately fitted by minimizing the residual in the spectrum in the methyl and allylic + alpha-carboxyl region specifically. 
The signal amplitude ratio of the diallylic over the methyl peaks and the ratio of the alphacarbonyl + allylic over the methyl peaks was determined in both the phantom and the HR experiment, to empirically determine correction factors for TE-induced signal in our STEAM recordings. These empirically determined correction factors were then used for the measurements in adipose tissue and in the liver to calculate the lipid composition using the following formulas:

$$
\begin{gathered}
\% P U F A=C A *\left(\frac{2}{3} * \frac{\text { Sdiallylic }}{\text { Smethyl }}\right) * 100 \\
\% M U F A=C B *\left[\frac{3}{4} *\left(\frac{\text { Salphacarb }+ \text { Sallylic }}{\text { Smethyl }}-\frac{2}{3}\right) * 100\right]-\% P U F A \\
\% S F A=100-\% P U F A-\% M U F A
\end{gathered}
$$

with $C A$ and $C B$ the empirically determined correction factor for the PUFA and MUFA calculation respectively ( $C A=0.83$ and $C B=0.99$; see also supplementary table 1 ).

In a third step, the lipid composition in subcutaneous adipose tissue was determined in vivo with ${ }^{1} \mathrm{H}-\mathrm{MRS}$ using the developed fitting routine and this was compared to the ex vivo lipid composition as determined by mass spectrometry analysis in adipose tissue biopsies, in eight participants. For the adipose tissue ${ }^{1} \mathrm{H}$-MRS measurement, we applied a gradient cycled STEAM sequence, to correct for eddy currents, as previously described (22).

In a final validation step to determine reproducibility, we applied the developed methodology in the liver of seven healthy individuals (BMI $30.6 \pm 3.7 \mathrm{~kg} / \mathrm{m}^{2}$; age $49 \pm 17.2$ year; 3 women), with a wide range of intrahepatic fat (2.1-19.6\%). To this end, we performed the ${ }^{1} \mathrm{H}-\mathrm{MRS}$ acquisition and repeated this after replacement of the subject on the table. The ICC was calculated for the lipid composition and total lipid content as a measure to determine the reproducibility between two consecutive measurements. Lipid content was calculated after $\mathrm{T}_{2}$ correction as ratio of the $\mathrm{CH}_{3}$ peak relative to the unsuppressed water resonance, expressed as percentage weight/weight.

\section{Deuterated water measurement of DNL}

A background blood sample was drawn in the afternoon before intake of the deuterated water in 18 healthy overweight/obese participants. Together with the evening meal, participants were given $2.86 \mathrm{~g} / \mathrm{kg}$ body weight deuterated water $(70 \% 2 \mathrm{H} 2 \mathrm{O}$, Cambridge Isotope laboratories) in two servings. A blood sample for DNL analysis was drawn at fasting in the morning, 16 hours after the first serving of deuterated water. The DNL was analyzed 
from enrichment in VLDL-TG of deuterated water $(10,23)$. Two participants were excluded from the analysis of DNL because of TG levels higher than $4 \mathrm{mmol} / \mathrm{L}$, influencing the reliability of the deuterated water measurement.

\section{Body composition}

Body mass and body volume were assessed using air-displacement plethysmography (ADP) using the Bod Pod device (Cosmed, Italy, Rome) according to the manufacturer's instructions on the day of the hyperinsulinemic-euglycemic clamp (healthy overweight/obese volunteers: $n=21$, patients with type 2 diabetes: $n=9)(24)$. Thoracic gas volume was predicted based on equations included in the Bod Pod software (version 4.2.0). From these data, body fat percentage was calculated as described by Siri (25).

\section{Subcutaneous adipose tissue biopsy}

In a subgroup of eight healthy overweight/obese participants, a subcutaneous adipose tissue biopsy ( $1 \mathrm{~g}$ ) was collected $6-8 \mathrm{~cm}$ lateral from the umbilicus, under local anesthesia ( $1 \%$ lidocaine) by needle biopsy before the start of the hyperinsulinemic-euglycemic clamp. Upon tissue collection, the tissue was rinsed with sterile saline and visible blood vessels were removed. Thereafter, the biopsy was snap-frozen in liquid nitrogen and stored at $80^{\circ} \mathrm{C}$ for later lipid composition analyses.

\section{Analysis of VLDL and adipose tissue triglycerides}

Plasma samples used for VLDL-TG analyses were taken around $16 \mathrm{pm}$ before the intake of the deuterated water. The VLDL fractions (20-50 $\mu \mathrm{l})$ and adipose tissues (10-20mg) were extracted using the BUME method $(26,27)$. The total lipid extracts were evaporated under a stream of nitrogen and reconstituted in $250 \mu \mathrm{L}$ chloroform/methanol [2:1]. Triglycerides were detected by direct infusion (shotgun) analysis on a QTRAP 5500 mass spectrometer (Sciex, Concord, Canada) equipped with a robotic nanoflow ion source, TriVersa NanoMate (Advion BioSciences, Ithaca, NJ) according to previous work (28). More specifically, a fraction of the total lipid extract was diluted 1:100 (for VLDL) and 1:100 000 (for adipose tissue) in chloroform:methanol [1:2] with $5 \mathrm{mM}$ ammonium acetate and infused at $250 \mathrm{nl} / \mathrm{min}$ for 15 minutes. The NanoMate was run with a voltage of $1.2 \mathrm{kV}$ and a gas pressure of 0.8 psi. The analysis was performed in positive ion mode by neutral loss detection of 11 common acyl fragments formed during collision induced dissociation of the ammoniated triglycerides. The nano-interface of the mass spectrometer was heated to 60 degrees and the measurements was made using a scan speed of 200 dalton/sec. The neutral loss scans 
were cycled and in total 50 cycles were acquired for each neutral loss scan (one neutral loss for each fatty acid). The data was processed using the LipidView 1.2 software (Sciex, Concord, Canada) and the most abundant signal intensities from the most commonly found triglyceride species were used to calculate the abundance and composition of the different triglycerides (supplementary table 2 and 3). Quantification was made using a one point calibration against glyceryl- $d_{5}$-hexadecanoate (CDN isotopes, Quebec, Canada), which was added to the infusion solvent.

\section{Hyperinsulinemic-euglycemic clamp}

A two-step hyperinsulinemic-euglycemic clamp was performed to assess hepatic insulin sensitivity using the low-dose phase $\left(10 \mathrm{mU} / \mathrm{m}^{2} / \mathrm{min}\right)$ (healthy overweight/obese participants: $n=21)$. A primed continuous infusion of $D-[6,6-2 \mathrm{H} 2]$ glucose $(0.04 \mathrm{mg} / \mathrm{kg} / \mathrm{min})$ was started to determine rates of endogenous glucose production (EGP), glucose appearance ( $\mathrm{Ra})$, and glucose disposal (Rd). After $180 \mathrm{~min}$, participants were given infusion of low insulin ( $10 \mathrm{mU} / \mathrm{m} 2 / \mathrm{min}$ ) for $3 \mathrm{~h}$. During the last $30 \mathrm{~min}$ of the insulin infusion step, blood samples were collected. Steele's single pool non- steady state equations were used to calculate glucose $\mathrm{Ra}$ and $\mathrm{Rd}$ (29). Volume of distribution was assumed to be $0.160 \mathrm{~L} / \mathrm{kg}$ for glucose. Hepatic insulin sensitivity was calculated as the percentage of EGP suppression during the low-dose phase. In addition, hepatic insulin sensitivity was determined according to this methodology in patients with type 2 diabetes $(n=9)$. Clamp parameters are shown in supplementary table 4.

\section{Statistical analysis}

Results are expressed as mean \pm SEM for group comparisons. Population characteristics are expressed as mean \pm SD. Continuous variables were tested for normality. Two-sided pearson correlation was performed to identify correlations between variables. For nonnormally distributed data two-sided spearman correlation was performed. Group comparisons were assessed by one-way ANOVA. For non-normally distributed data KruskalWallis analyses was performed to compare groups. In case of significant group differences in the group comparisons, post-hoc analyses were performed using Bonferroni correction to test which groups were significantly different. A p-value $<0.05$ was considered statistically significant. Statistical analyses were performed using SPSS 23.0 for Mac OS. 


\section{RESULTS}

\section{Development and validation of hepatic ${ }^{1} \mathrm{H}-\mathrm{MRS}$ method}

To allow determination of hepatic fatty acid composition, we developed a ${ }^{1} \mathrm{H}-\mathrm{MRS}$ analysis protocol. To this end, we used information from ${ }^{1} \mathrm{H}$ high-resolution (HR) NMR spectra from five different vegetable oils to improve our analysis routine and used ${ }^{13} \mathrm{C} H R$ NMR to determine the true lipid composition of these oils. We acquired spectra from these different oils with proton magnetic resonance spectroscopy $\left({ }^{1} \mathrm{H}-\mathrm{MRS}\right)$ on our clinical $3 \mathrm{~T}$ scanner and calculated average corrections factors to correct for TE-induced losses in the ${ }^{1} \mathrm{H}-\mathrm{MRS}$ spectra. When using the average corrections factors, the lipid composition as determined by our clinical protocol showed excellent agreement with the true lipid composition determined by high-resolution NMR: the intraclass correlation coefficient (ICC) for SFA, MUFA and PUFA fraction was $0.982,0.970,0.987$ and the CV was $6 \%, 9 \%$ and $9 \%$, respectively (figure 1 ).

As it is known that in vivo several factors can influence MR spectra, the next step was to validate our method in vivo. To this end, we aimed to validate our ${ }^{1} \mathrm{H}$-MRS lipid composition measurement with analysis performed in biopsy material. As it is ethically difficult to take liver biopsies for this purpose, we rather performed subcutaneous adipose tissue biopsies, which is far less invasive. Therefore, we validated our MRS method in vivo by comparing lipid composition in subcutaneous adipose tissue acquired by the ${ }^{1} \mathrm{H}-\mathrm{MRS}$ technique and by mass spectrometry analysis in subcutaneous adipose tissue biopsies in eight participants (figure 2). As can be seen in figure 2, there was reasonably good agreement between the two methods. The ICC for SFA, MUFA and PUFA fraction was $0.333,0.146,0.306$ and the CV was $8 \%, 7 \% ; 12 \%$, respectively. Thus, lipid composition determined by ${ }^{1} \mathrm{H}-\mathrm{MRS}$ in vivo is in close agreement with lipid composition measured ex vivo in adipose tissue biopsies. The final validation step was to apply our protocol in the liver in vivo. We tested reproducibility in seven healthy individuals (separate group) with intrahepatic lipid content ranging from $2-18 \%$ (figure 3). As can be seen in figure 3, the ICC for total IHL content, SFA, MUFA and PUFA fraction was found to be $0.997,0.562,0.756,0.557$ and the CV was $4 \%, 4 \%, 3 \%$, and $12 \%$, respectively. These results indicate high reproducibility for determining in vivo hepatic lipid composition using our developed approach.

In addition, we compared hepatic lipid composition by our MRS method to plasma VLDLtriglyceride (VLDL-TG) composition acquired by mass spectrometry analysis in seventeen participants (figure 4). As can be seen in figure 4, especially the SFA fraction showed a strong correlation with hepatic SFA\% (Pearson $r=0.80, p<0.001$ ). 


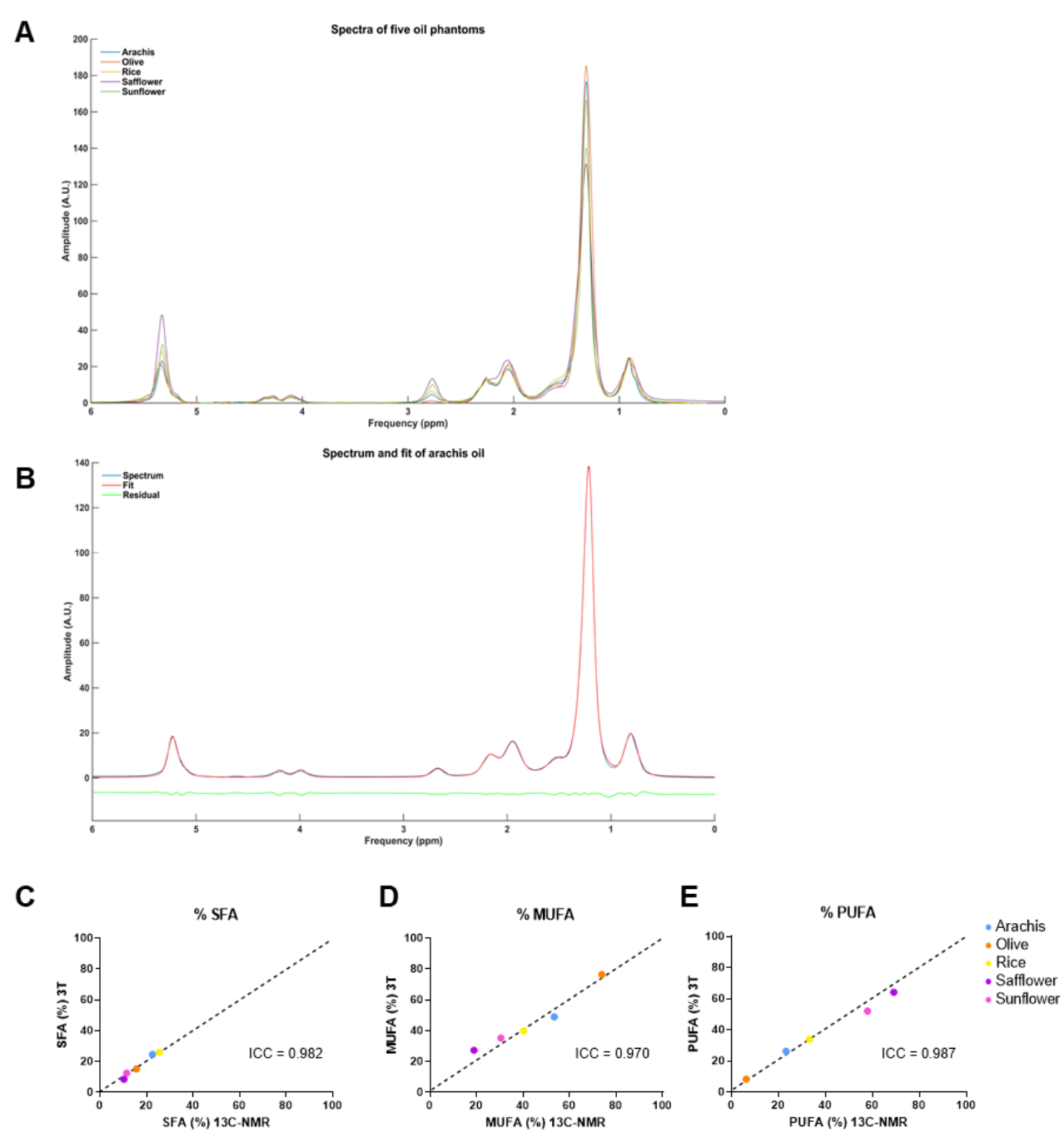

Figure 1: Validation of ${ }^{1} \mathrm{H}-\mathrm{MRS}$ method in oil phantoms. A Lipid spectra acquired from five different oil phantoms (arachis, olive, rice, safflower and sunflower oil) showing the different lipid proton peaks and their position. B Lipid spectrum and fit of arachis oil. Correlations between C SFA, D MUFA, E PUFA measured at $3 T$ with ${ }^{1} \mathrm{H}-\mathrm{MRS}$ and measured with high-resolution ${ }^{13} \mathrm{C}-\mathrm{NMR}$ spectroscopy. The intraclass correlation coefficient (ICC) is shown in the respective plots (Intraclass correlation). 


\section{Chapter 3}

A
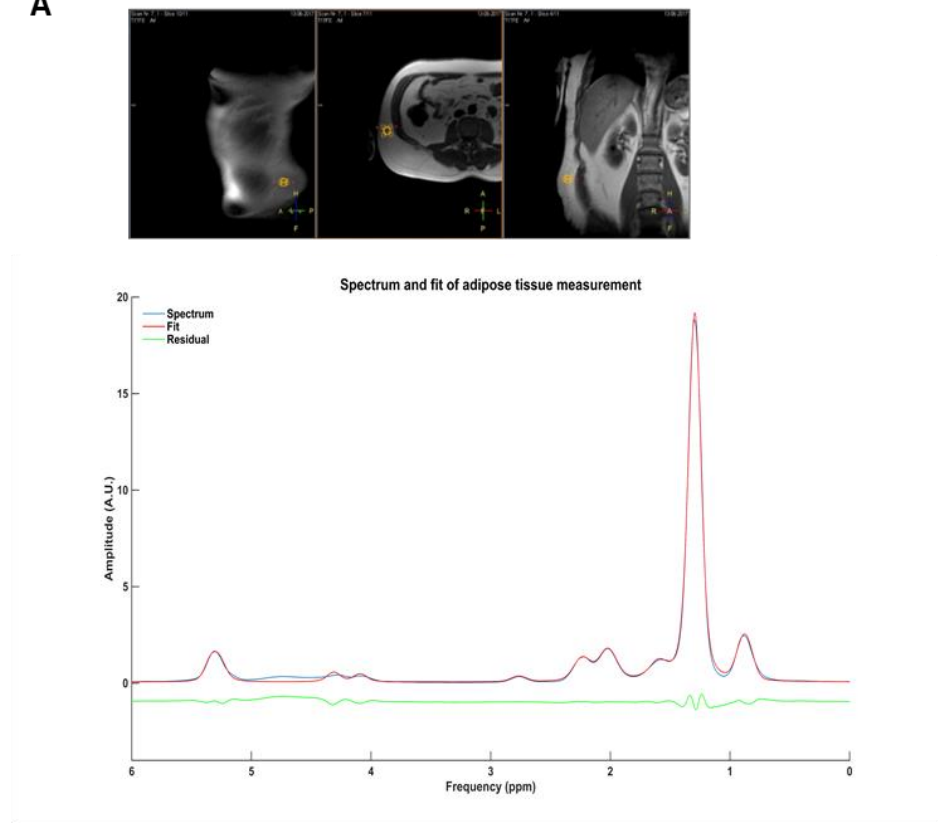

B

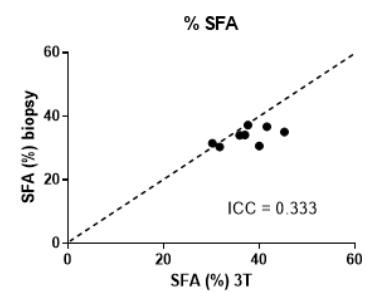

C

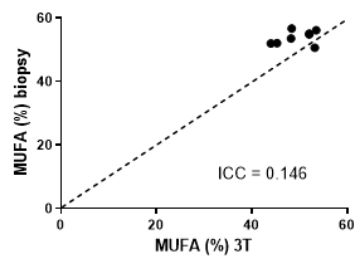

D

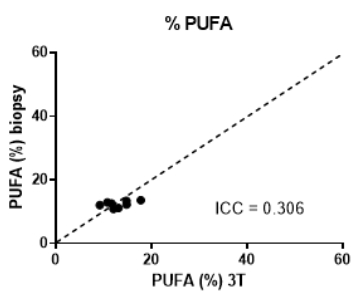

Figure 2: Validation of ${ }^{1} \mathrm{H}$-MRS method in subcutaneous adipose tissue. A T2 weighted Turbo spin echo MR image showing the voxel position located on adipose tissue and its corresponding lipid spectrum together with the fitted spectrum. The relationships between subcutaneous adipose tissue measured at $3 \mathrm{~T}$ and adipose lipid composition determined through biopsy for the different lipid fractions: B SFA, C MUFA and D PUFA ( $n=8)$. The intraclass correlation coefficient (ICC) is shown in the respective plots (Intraclass correlation). 
A
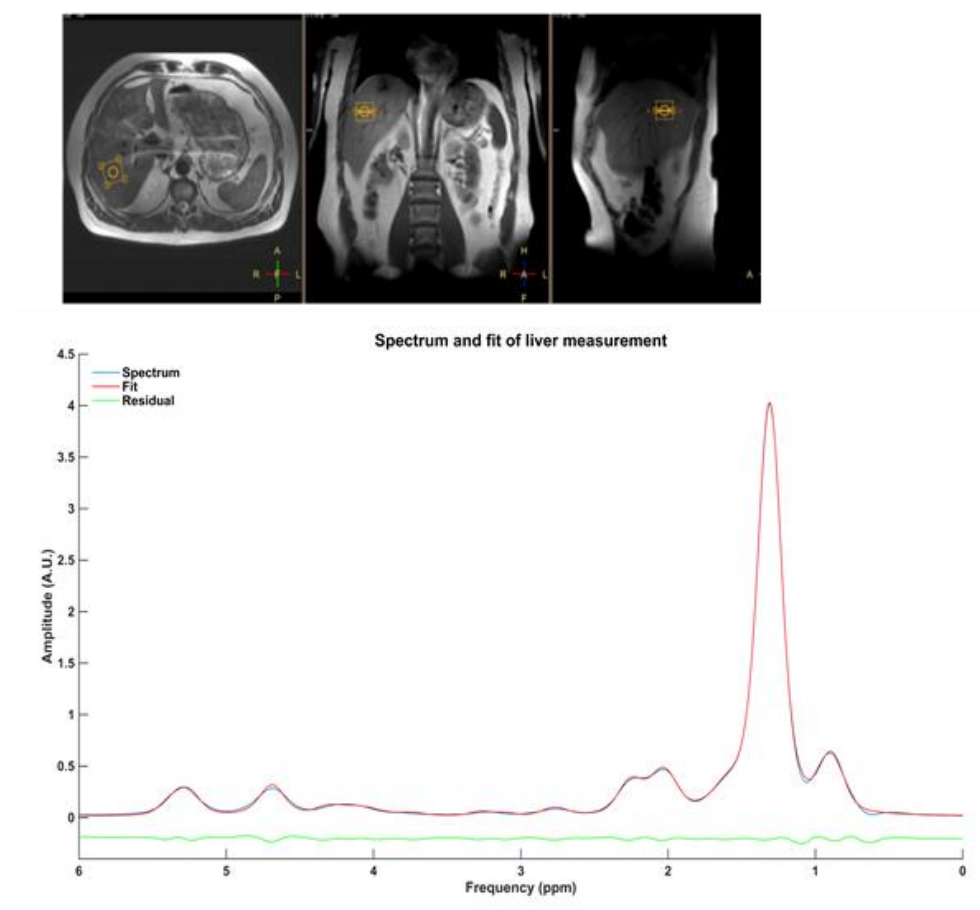

B

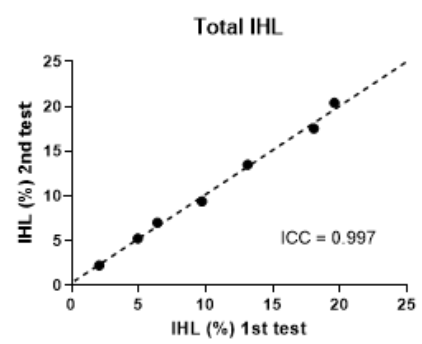

D

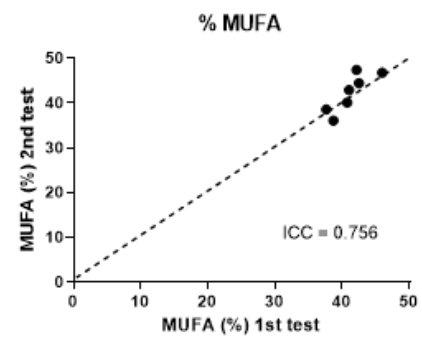

C

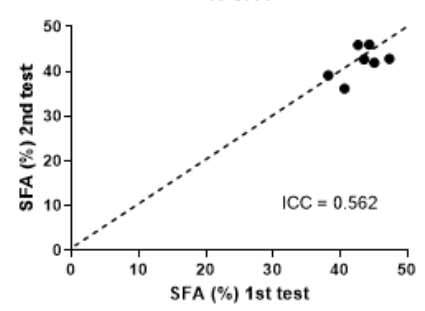

E

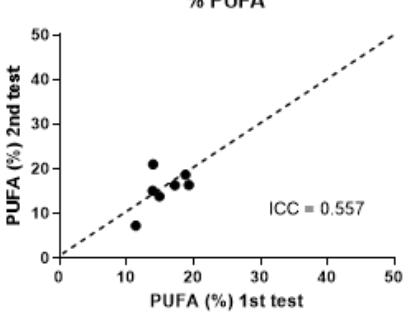

Figure 3: Validation of ${ }^{1} \mathrm{H}-\mathrm{MRS}$ method by testing reproducibility in vivo. A T2 weighted Turbo spin echo MR image showing the voxel position located on liver and its corresponding lipid spectrum together with the fitted spectrum. Scatter plots showing the reproducibility of B total liver fat content and C SFA fraction, D MUFA fraction and E PUFA fraction. ( $n=7)$. Reproducibility was tested by performing two repeated measurements. The intraclass correlation coefficient (ICC) is shown in the respective plots (Intraclass correlation). 
A

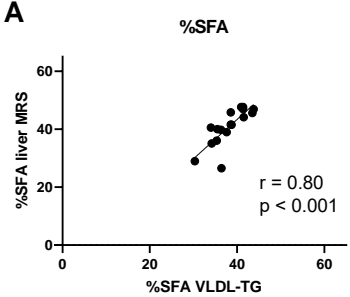

B

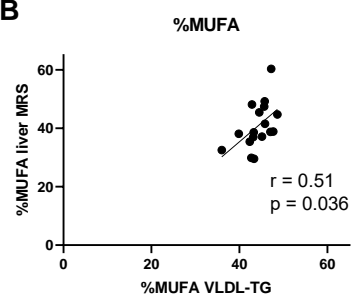

C

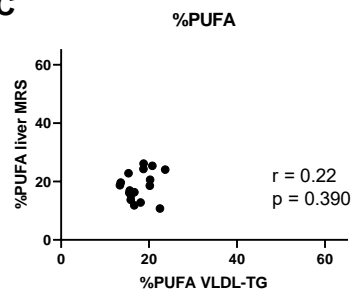

Figure 4: The relationship between hepatic lipid composition and plasma VLDL-TG composition. Relationships are shown for the different lipid fractions: A SFA, B MUFA and C PUFA ( $n=17)$. Hepatic \%SFA determined with MRS and \%SFA in VLDL-TG correlated significantly $\left(p=1.38 \times 10^{-4}\right)$. The correlation coefficient is shown in the respective plots (two-sided Pearson correlation).

\section{Higher hepatic SFA is associated with increased DNL}

DNL is an important factor in the development of fatty liver (30). We hypothesized that DNL would specifically lead to the accumulation of saturated fatty acids, as palmitate is the main product of DNL. Therefore, we determined DNL by deuterated water in overweight and obese participants with a wide range of liver fat content $(n=16,0.9-38.4 \%)$ and related it to the hepatic fatty acid composition determined by our MRS protocol in the same volunteers. DNL was not associated with total liver fat content (figure 5A). Interestingly, however, DNL correlated positively with the hepatic SFA fraction (Pearson $r=0.52, p=0.047$; figure 5B). Furthermore, we found a strong negative correlation between DNL and hepatic MUFA fraction (Pearson $r=-0.71, p=0.003$; figure $5 C$ ). In addition, $D N L$ was negatively correlated with MUFA/SFA ratio (Pearson $r=-0.64 p=0.010$; figure $5 E$ ). No association was found between DNL and PUFA fraction (figure 5D). Of note, SFA fraction in plasma VLDL-TG did not correlate with DNL (Pearson $r=0.27, p=0.308$ ). These results suggest that 1 ) higher rates of DNL lead to altered saturation of hepatic lipids, and 2) that our non-invasive method can be used as a measure of the degree of DNL, directly in the liver. 
A

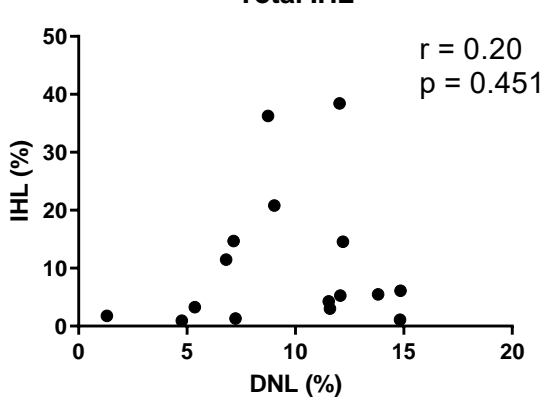

C

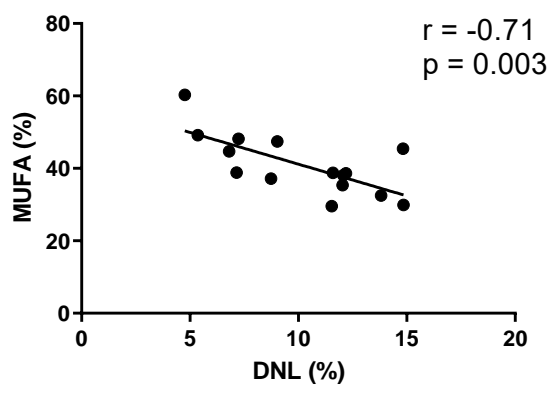

E

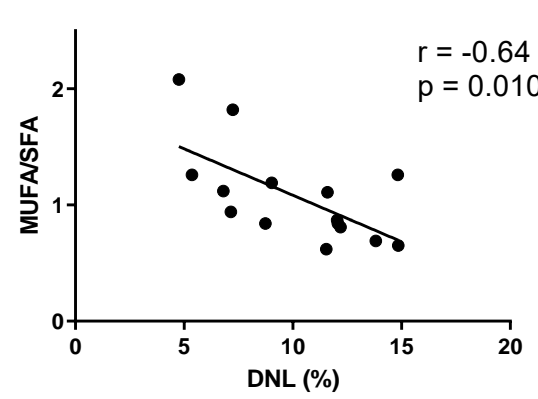

B

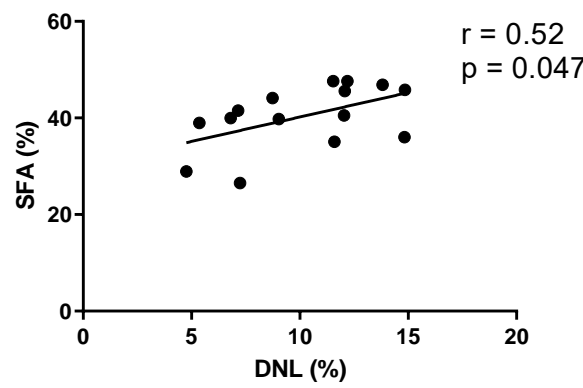

D

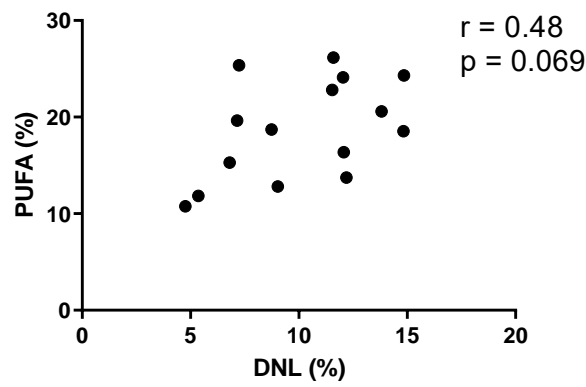

Figure 5: Relationship between DNL and liver fat composition. The relationships between DNL and $\mathbf{A}$ total liver fat content, B SFA fraction, C MUFA fraction, D PUFA fraction and E MUFA/SFA ratio in healthy overweight/obese participants (with and without NAFL, $n=16$ for total liver fat content and $n=15$ for the fatty acid fractions and MUFA/SFA ratio). The correlation coefficient is shown in the respective plots ( $\mathrm{A}$; two-sided Spearman correlation, B-E; two-sided Pearson correlation). 


\section{Hepatic SFA fraction is of clinical relevance in humans}

To investigate if hepatic lipid composition may also have clinical relevance, we compared hepatic lipid composition in volunteers with a range in metabolic complications. We subdivided the volunteers in whom we demonstrated the relationship between DNL and hepatic SFA, into participants with NAFL (liver fat content $>5 \%, n=15$ ) and control participants (liver fat content $<5 \%, n=7$ ); volunteers with NAFL are known to be at increased risk to develop type 2 diabetes and other cardiometabolic disorders (6-8). In addition, we measured hepatic lipid fractions and hepatic insulin sensitivity (IS) in a group of patients with type 2 diabetes $(n=9)$ and furthermore, determined hepatic lipid fractions in glycogen storage disease type 1a (GSD1a) patients $(n=7)$, known to have elevated rates of DNL. This inborn error of metabolism is caused by a defect in glucose-6-phosphatase, which hampers the final step in gluconeogenesis and glycogenolysis in liver and kidney, and, as a consequence, favours the shift of glucose-6-phosphate towards DNL and hepatic steatosis (31). Control and NAFL participants and patients with type 2 diabetes were comparable in age and BMI. All subject characteristics are summarized in table 1.

Table 1: Subject characteristics of control and NAFL participants, patients with T2D and GSD1a.

\begin{tabular}{lcccc}
\hline & Control $(\mathrm{n}=7)$ & NAFL $(\mathrm{n}=15)$ & T2D $(\mathrm{n}=9)$ & GSD1a $(\mathrm{n}=7)$ \\
\hline Age (years) & $59 \pm 6.8$ & $58 \pm 7.1$ & $65 \pm 4.5$ & $37 \pm 12.3^{\mathrm{A}, \mathrm{B}, \mathrm{C}}$ \\
BMI (kg/m $\left.{ }^{2}\right)$ & $29.1 \pm 2.3$ & $30.7 \pm 3.1$ & $29.4 \pm 4.2$ & $27.5 \pm 3.2$ \\
Sex (f/m) & $6 / 1$ & $7 / 8$ & $2 / 7$ & $5 / 2$ \\
Body fat (\%) & $42.1 \pm 8.1$ & $42.1 \pm 7.8$ & $34.1 \pm 5.8$ & - \\
Plasma glucose (mmol/L) & $5.2 \pm 0.4$ & $5.6 \pm 0.5$ & $7.5 \pm 1.1^{\mathrm{A}, \mathrm{B}}$ & $3.9 \pm 0.8^{\mathrm{A}, \mathrm{B}, \mathrm{C}}$ \\
Plasma insulin (pmol/L) & $35.2 \pm 8.1$ & $89.8 \pm 40.4^{\mathrm{A}}$ & $72.8 \pm 58.3$ & $13.8 \pm 5.0^{\mathrm{B}, \mathrm{C}}$ \\
Plasma NEFA (mmol/L) & $667 \pm 58$ & $623 \pm 141$ & $566 \pm 211$ & $876 \pm 421$ \\
Plasma TG (mmol/L) & $1.5 \pm 0.8$ & $2.6 \pm 1.2$ & $1.6 \pm 0.5$ & $5.0 \pm 1.5^{\mathrm{A}, \mathrm{B}, \mathrm{C}}$ \\
ALT (U/L) & $22 \pm 4.1$ & $35 \pm 14.4$ & $27 \pm 11.4$ & $18 \pm 4.9^{\mathrm{B}}$ \\
AST (U/L) & $22 \pm 3.7$ & $28 \pm 6.3$ & $23 \pm 5.9$ & $34 \pm 9.3^{\mathrm{A}, \mathrm{C}}$ \\
Intrahepatic fat content & $2.2 \pm 1.3$ & $14.4 \pm 10.4^{\mathrm{A}}$ & $6.9 \pm 5.2$ & $16.5^{\mathrm{C}} \pm 18.9^{\mathrm{A}}$ \\
(\% weight/weight) & & & & \\
\hline
\end{tabular}

Data are presented as mean \pm SD. Overweight/obese controls without NAFL (controls, $n=7$ ), overweight/obese with NAFL (NAFL, $n=15$ ), patients with type 2 diabetes (T2D, n=9) and GSD type 1a (GSD1a, n=7). Age was significantly lower in GSD1a compared to control $\left(p=2.5 \times 10^{-5}\right)$, NAFL $\left(p=3.0 \times 10^{-6}\right)$ and T2D $\left(p=1.47 \times 10^{-7}\right)$. Plasma glucose was significantly lower in GSD1a compared to control $(p=0.011)$, NAFL $\left(p=5.6 \times 10^{-5}\right)$ and T2D $\left(p=1.44 \times 10^{-}\right.$ $\left.{ }^{10}\right)$, and was significantly higher in T2D compared to control $\left(p=4.0 \times 10^{-6}\right)$ and NAFL $\left(p=6.0 \times 10^{-6}\right)$. Plasma insulin was significantly lower in GSD1a compared to NAFL $\left(p=3.4 \times 10^{-5}\right)$ and T2D $(p=0.006)$, and was significantly higher in NAFL compared to control $(p=0.048)$. Plasma TG was significantly higher in GSD1a compared to control $\left(p=3.0 \times 10^{-6}\right)$, NAFL $\left(p=1.33 \times 10^{-4}\right)$ and T2D $\left(p=2.0 \times 10^{-6}\right)$. ALT was significantly higher in NAFL compared to GSD1a $(p=0.011)$. AST was significantly higher in GSD1a compared to control $(p=0.006)$ and T2D ( $p=0.017)$. Intrahepatic fat content was significantly higher in NAFL compared to control $(p=0.002)$ and GSD1a compared to control $(p=0.027)$. A Significantly different from control, ${ }^{B}$ significantly different from NAFL, ${ }^{\mathrm{C}}$ significantly different from T2D (KruskalWallis, $p<0.05$ for plasma insulin, plasma NEFA, ALT and intrahepatic fat content, and one-way ANOVA, $p<0.05$ for all other parameters). Bonferroni correction was used for post-hoc analyses. 
Hepatic SFA fraction was higher in NAFL individuals $(42.9 \% \pm 0.8 \%$, ANOVA, $p=0.022$, mean \pm SEM; figure $6 \mathrm{~B})$ and in type 2 diabetes patients $(43.9 \% \pm 1.7 \%$, ANOVA, $p=0.016$; figure $6 \mathrm{~B})$ compared to controls $(35.5 \% \pm 3.1 \%)$. Hepatic MUFA and PUFA fractions did not significantly differ between these groups (figure 6C and 6D). No significant associations were found between hepatic fat content and any of the FA fractions in healthy participants with and without NAFL. Therefore, these data suggest that specifically the SFA fraction is elevated in metabolically compromised volunteers.

To further confirm that DNL and SFA fraction may be causally related, and to further investigate the clinical relevance of SFA, we determined SFA fraction in a specific group of patients, known to have elevated rates of DNL, i.e. patients with glycogen storage disease type 1a (GSD1a) ( $n=7)$. In these patients, SFA fraction $(49.5 \% \pm 2.0 \%)$ was markedly higher compared to NAFL participants $(42.9 \% \pm 0.8 \%$, ANOVA, $p=0.034$; figure $6 \mathrm{~B})$ and controls $(35.5 \% \pm 3.1 \%$, ANOVA, $p<0.001$; figure $6 \mathrm{~B})$. The MUFA fraction in these patients was reduced compared to controls ( $33.2 \% \pm 1.1 \%$ vs. $45.2 \% \pm 4.2 \%$, ANOVA, $p=0.006$; figure $6 C$ ). These results further confirm that the fraction of hepatic SFA and MUFA reflect the rate of DNL and can be used as a non-invasive measure to characterise hepatic metabolism in clinical patients in more detail.

\section{Higher hepatic SFA is associated with reduced hepatic IS}

Our results so far suggest that specifically the saturated fat fraction is elevated in patients and individuals with an enhanced metabolic risk. These data are consistent with findings in animal studies, where it was found that specifically hepatic SFA is of importance in relation to insulin resistance (13). We therefore investigated the relationship between hepatic lipid composition and hepatic IS using the golden-standard two-step hyperinsulinemic euglycemic clamp. Hepatic IS was found to be $40 \%$ lower in patients with type 2 diabetes compared to controls (EGP suppression controls: $74.2 \% \pm 7.0 \%$ and T2D: $41.6 \% \pm 4.1 \%$, ANOVA, $p=0.002$; figure 7F), with intermediate values in NAFL (EGP suppression $56.9 \% \pm$ 4.9\%; figure 7F). The hepatic SFA fraction was strongly and negatively associated with hepatic IS (Spearman $r=-0.55, p=0.002$; figure 7B), whereas correlation with hepatic MUFA fraction was weaker (Spearman $r=0.39, p=0.034$; figure $7 C$ ) and PUFA fraction and total hepatic fat content did not correlate with hepatic IS (figure 7A, D). Furthermore, the MUFA/SFA ratio was positively associated with hepatic IS (Spearman $r=0.51, p=0.005$; figure $7 E$ ). These results suggest that specifically the SFA fraction in the liver negatively contributes to hepatic IS, rather than the total amount of hepatic fat. 
A

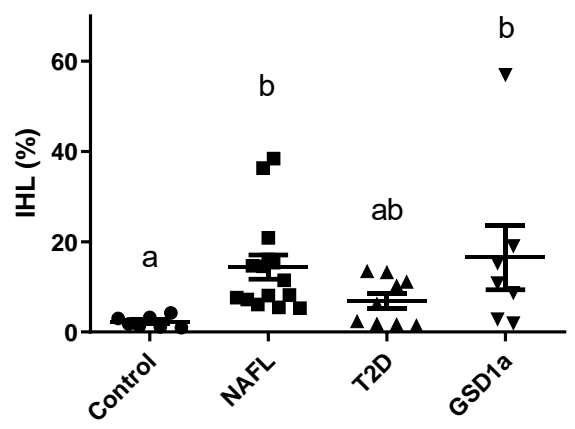

C

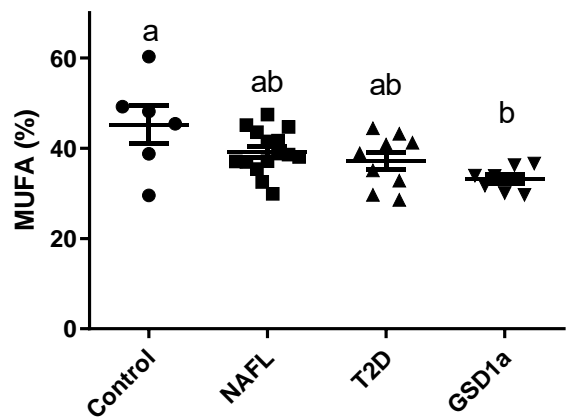

B

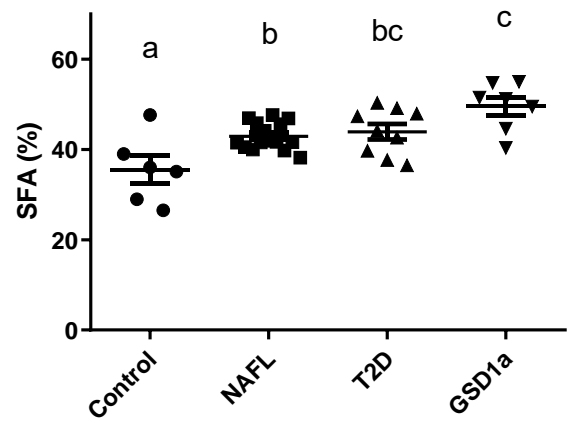

D

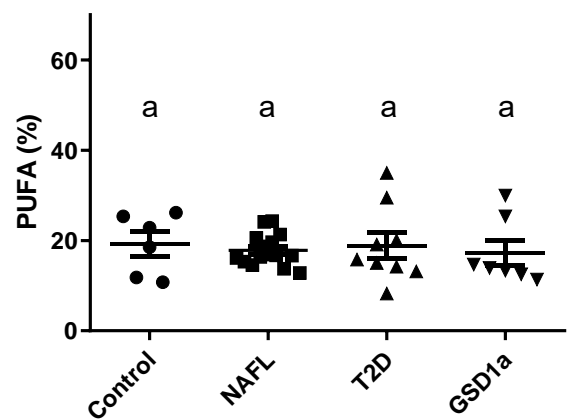

Figure 6: Liver fat content and composition in groups with different metabolic disorders. Comparisons between overweight/obese controls without NAFL (controls, $n=7$ for total liver fat content, $n=6$ for liver fat composition), overweight/obese with NAFL (NAFL, $n=15$ ), patients with type 2 diabetes (T2D, n=9) and GSD type 1a (GSD1a, $n=7)$. A Total liver fat content in control, NAFL, T2D and GSD1a. Total liver fat content was significantly higher in the NAFL group compared to the control group $(p=0.002)$ and in the GSD1a group compared to the control group $(p=0.027)$. B SFA fraction in control, NAFL, T2D and GSD1a. SFA fraction was significantly higher in the GSD1a group compared to the control group $\left(p=7.3 \times 10^{-5}\right)$ and NAFL group $(p=0.034)$, significantly higher in the T2D group compared to the control group ( $p=0.016$ ), and significantly higher in the NAFL group compared to the control group $(p=0.022)$. C MUFA fraction in control, NAFL, T2D and GSD1a. MUFA fraction was significantly lower in the GSD1a group compared to the control group $(p=0.006)$. D PUFA fraction in control, NAFL, T2D and GSD1a. Data are presented as mean with error bars showing the SEM. Different letters indicate significant differences between groups (Kruskal-Wallis, $\mathrm{p}<0.05$ for IHL and PUFA, and one-way ANOVA, $\mathrm{p}<0.05$ for SFA and MUFA). Bonferroni correction was used for post-hoc analyses. 

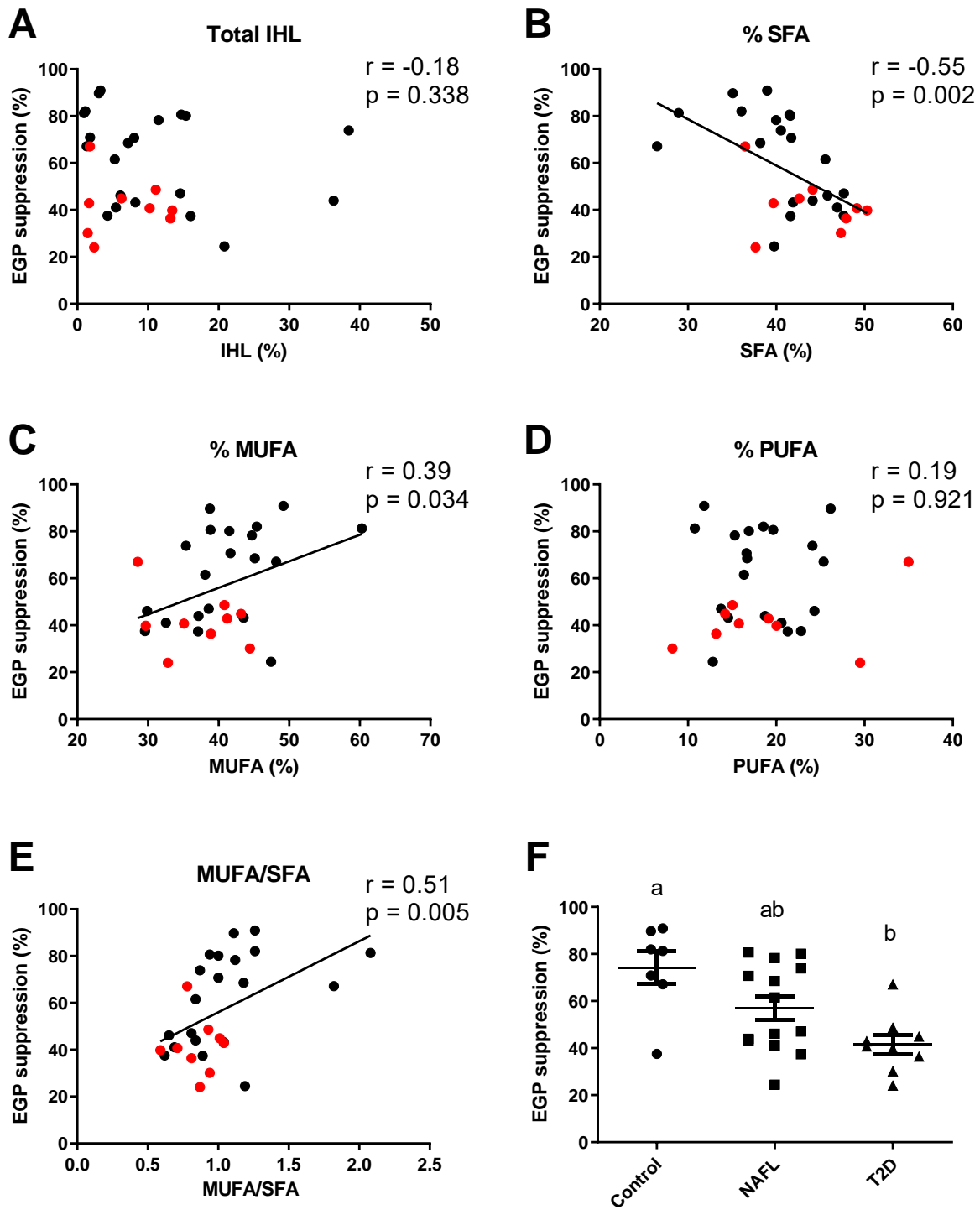

Figure 7: The relationship between liver fat composition and hepatic insulin sensitivity. The relationship between hepatic IS (EGP suppression) and A total liver fat content, B SFA fraction, C MUFA fraction and D PUFA fraction and E MUFA/SFA ratio in overweight and obese individuals (healthy with and without NAFL in black and patients with T2D in red, $n=30$ for total liver fat content and $n=29$ for the fatty acid fractions and MUFA/SFA ratio). The correlation coefficient is shown in the respective plots (Two-sided Spearman correlation). F EGP suppression in healthy overweight/obese without NAFL (controls, $n=7)$, overweight/obese with NAFL $(n=14)$ and patients with T2D ( $n=9)$. EGP suppression in patients with T2D was significantly lower compared to controls $(p=0.002)$. Data are presented as mean with error bars showing the SEM. Different letters indicate significant differences between groups (one-way ANOVA, $\mathrm{p}<0.05$ ). Bonferroni correction was used for post-hoc analyses. 


\section{DISCUSSION}

The relation of hepatic lipid composition to cardiometabolic health and specifically insulin resistance has not been investigated in humans. Due to the invasive procedures necessary and due to challenges accompanying the use of non-invasive methods such as ${ }^{1} \mathrm{H}-\mathrm{MRS}$, determination of hepatic lipid composition is hampered. Here, we developed, validated and applied ${ }^{1} \mathrm{H}-\mathrm{MRS}$ methodology that enabled us to non-invasively quantify the fractions of hepatic SFA, MUFA and PUFA separately. We showed that DNL was positively related to the hepatic SFA fraction and negatively to the MUFA fraction in overweight/obese participants, suggesting that high rates of DNL modify fatty acid composition. We confirmed this association between DNL and hepatic SFA fraction by showing increased hepatic SFA fraction in GSD1a patients, a genetic model of increased DNL. Furthermore, we showed that the hepatic SFA fraction is elevated in NAFL and type 2 diabetes patients and related to hepatic insulin resistance. These results show that our ${ }^{1} \mathrm{H}-\mathrm{MRS}$ protocol can be used to determine hepatic fat composition in humans and that particularly the SFA fraction can be seen as a non-invasive measure of DNL, directly measured in the liver. Furthermore, our results indicate that hepatic lipid composition may be a clinically important feature of hepatic fat accumulation.

Previously, a few studies have used similar approaches using ${ }^{1} \mathrm{H}$-MRS for hepatic lipid profiling, but none of these studies have reported SFA, MUFA and PUFA fractions separately (16-19). Even more so, in case of changes in MUFA/PUFA ratios, the calculation of the saturation index by using the methine resonance can lead to a misinterpretation of the lipid composition. We here show that by acquiring high quality liver spectra and by applying a sophisticated post-processing method for these spectra it is possible to differentiate between the hepatic SFA, MUFA and PUFA fractions in vivo in subjects with and without NAFL. Implementation of this methodology in future studies makes it possible to further explore the importance of hepatic fat composition in metabolic health and the factors that could modulate fatty acid composition.

High rates of DNL may change hepatic lipid composition. The end product of the lipogenic pathway is palmitate (C16:0), which in turn can undergo elongation to stearate (C18:0) and desaturation by Stearoyl-CoA desaturase-1 (SCD1) to the MUFAs palmitoleate (C16:1 n-7) and oleate (C18:1 n-9). Previously, it has been shown that changes in DNL as determined by stable isotope techniques correlate with changes in the fraction of palmitate in VLDL-TG (32). In line with this, DNL was positively correlated with the hepatic SFA fraction in the current study. The hepatic MUFA fraction was negatively correlated with DNL, whereas the hepatic PUFA fraction did not correlate with DNL. From these results, it follows that upon 
increased DNL, the production of SFA is not necessarily accompanied by subsequent desaturation to MUFAs in humans. This is in contrast to animal studies that indicate that desaturation is upregulated in parallel to upregulation of lipogenic enzymes (13). In line, Peter et al. showed that SCD1 mRNA expression and C18:1/C18:0 ratio in TG and phospholipid fractions in human liver were not increased in individuals with NAFL (33). Thus, handling of fatty acids originating from DNL by the liver may be different in humans and animals. Increased desaturation following lipogenesis is believed to be a rescue mechanism to reduce the negative effects exerted by saturated fatty acids (13). Further, we find higher hepatic SFA fraction in patients with NAFL and T2DM compared to controls, which is in line with earlier results, showing that the relative contribution of DNL derived FA to VLDL-TG was around $5-10 \%$ in healthy individuals in the fasted state, whereas their contribution increased to around $20-25 \%$ in people with $\operatorname{NAFL}(11,30,34,35)$. These results suggest that the negative health effects that have been attributed to increased rates of DNL may also be due to the high fraction of SFA that result from DNL. Therefore, drug development targeting DNL is promising (36-38) although it is important to note that the evidence in the present study is only associative and future research will have to show whether decreasing the amount of SFA has beneficial effects on metabolic health. Interestingly however, animal studies support this notion. In mice overexpressing carbohydrate-responsive elementbinding protein (ChREBP), resulting in increased activity of SCD1 and higher MUFA fraction, the increased MUFA fraction at the expense of SFA fraction has been associated with increased insulin sensitivity, despite high amounts of total hepatic fat content (13). Furthermore, it has been shown in vitro that specifically SFA negatively affect insulin signaling and that desaturation of fatty acids can rescue these negative effects (13). In line with these animal and cell data, we here show that the hepatic SFA fraction was negatively correlated and the MUFA/SFA ratio positively correlated with hepatic insulin sensitivity in overweight and obese participants. Plasma (VLDL-)TG is often used as a surrogate for hepatic TG. In addition, with sophisticated centrifugation methods, saturation of plasma VLDL-TG can be determined. Here we showed that saturation of hepatic lipids correlated with the saturation in plasma VLDL-TG. Interestingly however, unlike the MRS determined SFA fraction, the SFA fraction in VLDL-TG did not correlate with DNL. These results indicate, that the SFA fraction determined by MRS is a better measure of DNL compared to the SFA fraction in VLDL-TG, and thus the use of our $1 \mathrm{H}-\mathrm{MRS}$ approach provides extra valuable information that could not be determined from plasma VLDL-TG.

In summary, we developed an MR protocol that enabled us to non-invasively quantify the fractions of hepatic SFA, MUFA and PUFA. Applying this ${ }^{1} \mathrm{H}-\mathrm{MRS}$ methodology, we here show that the hepatic SFA fraction is positively associated with DNL, is elevated in patients with NAFL and T2D, and negatively associated with hepatic insulin sensitivity. These results 
Chapter 3

indicate that hepatic lipid composition, as determined by our ${ }^{1} \mathrm{H}-\mathrm{MRS}$ methodology, might be used as measure of DNL and furthermore suggest that specifically the hepatic SFA fraction may determine metabolic health. 


\section{ACKNOWLEDGEMENTS}

These authors contributed equally: Kay Roumans, Lucas Lindeboom

K.R. performed the experiments, analysed data, and wrote the manuscript. L.L. was involved in design of experiments, has set up the post-processing pipeline and contributed to writing the manuscript. P.V. was involved in setting up the post-processing pipeline, performed experiments, analysed data and reviewed and edited the manuscript. C.R., E.P., B.H., Y.B., M.S. performed experiments and reviewed and edited the manuscript. M.B., M.A., H.P., R.M., B.S., M-R.T., J.B., P.S., V.S. were involved in the design of the study, interpretation of the data, and reviewed and edited the manuscript.

This research was in part financed by the Ministry of Economic Affairs and Climate Policy by means of the PPP Allowance made available by the Top Sector Life Sciences \& Health to stimulate public-private partnerships and by Unilever R\&D Wageningen. We acknowledge the support from the Netherlands Cardiovascular Research Initiative: an initiative with support of the Dutch Heart Foundation (CVON2014-02 ENERGISE). LL is a recipient of a veni grant from ZonMW (016.veni.188.036) and a junior fellowship (2017.81.004) from the Diabetes Fonds (Dutch Diabetes Research Foundation). BS is a recipient of an Advanced ERC Grant (694717). VS is a recipient of an ERC starting grant (grant no. 759161 'MRS in diabetes').

HP and MA were employees of Unilever at the time this research was designed and conducted and have since changed their professional affiliations. HP: Superfoods, Landsmeer. MA: Health Counsel, NL.

The authors declare no competing interests. 


\section{SUPPLEMENTARY MATERIAL FOR CHAPTER 3}

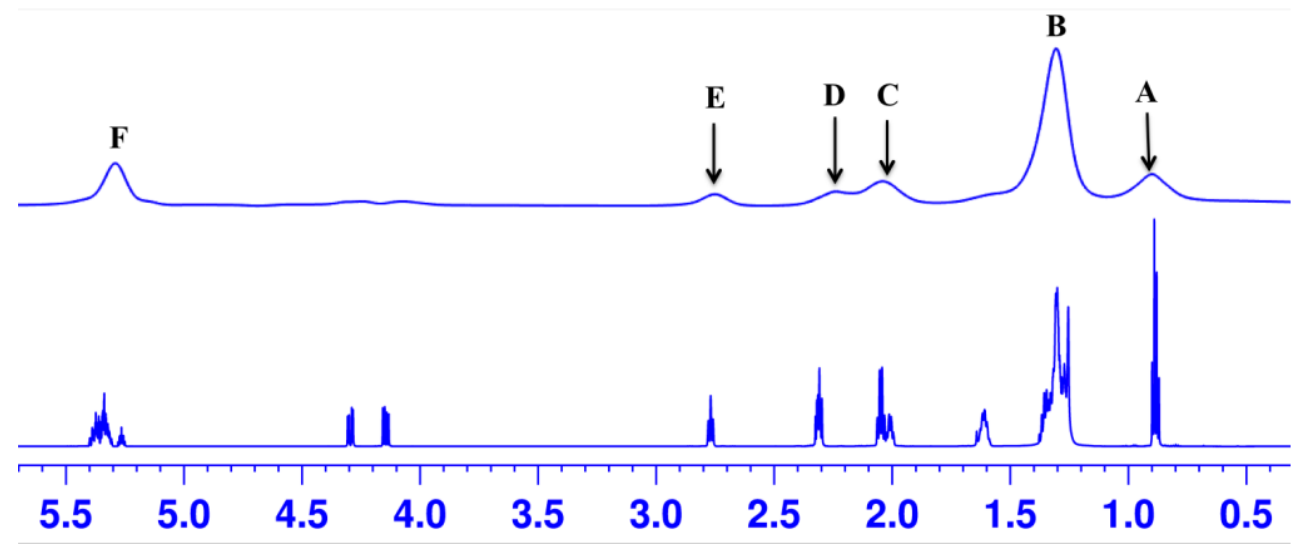

Supplementary figure 1: Comparison of lipid spectrum acquired at $3 \mathrm{~T}$ and high-resolution ${ }^{1} \mathrm{H}-\mathrm{NMR}$. Lipid spectrum acquired from the sunflower oil at $3 \mathrm{~T}$ (top) and high-resolution ${ }^{1} \mathrm{H}-\mathrm{NMR}$ spectrum (bottom) showing the variation in spectra resolution. A-methyl $\left(-\mathrm{CH}_{3}\right)$; B-methylene $\left(-\mathrm{CH}_{2}\right)$; C-allylic $\left(\mathrm{CH}_{3}-\mathrm{CH}=\mathrm{CH}-\mathrm{CH}\right)$; D-alpha carbonyl $\left(\mathrm{CH}_{2}-\mathrm{COO}\right)$; E-diallylic ( $\left.=\mathrm{CH}-\mathrm{CH}_{2}-\mathrm{CH}=\right)$; F-methine $(\mathrm{CH}=\mathrm{CH})$.

Supplementary table 1: Correction factors for PUFA and MUFA calculation

\begin{tabular}{lcc}
\hline & CA & CB \\
\hline Olive oil & 0,759 & 0,997 \\
Arachis oil & 0,735 & 0,987 \\
Sunflower oil & 0,907 & 1,003 \\
Safflower oil & 0,889 & 0,982 \\
Rice oil & 0,849 & 0,990 \\
\hline
\end{tabular}


Supplementary table 2: List of triglyceride species determined by mass spectrometry

\begin{tabular}{l} 
Triglyceride species \\
\hline TAG 48:0 \\
TAG 48:1 \\
TAG 48:2 \\
TAG 48:3 \\
TAG 50:0 \\
TAG 50:1 \\
TAG 50:2 \\
TAG 50:3 \\
TAG 50:4 \\
TAG 50:5 \\
TAG 52:0 \\
TAG 52:1 \\
TAG 52:2 \\
TAG 52:3 \\
TAG 52:4 \\
TAG 52:5 \\
TAG 52:6 \\
TAG 54:1 \\
TAG 54:2 \\
TAG 54:3 \\
TAG 54:4 \\
TAG 54:5 \\
TAG 54:6 $58: 8$ \\
TAG 54:7 $58: 9$ \\
TAG 56:3 $5: 4$ \\
TAG 56:5 $56: 6$ \\
TAG 58:7 \\
\hline
\end{tabular}

Supplementary table 3: List of fatty acid species determined by mass spectrometry

\begin{tabular}{ll}
\hline FA species & Saturation \\
\hline FA 14:0 & SFA \\
FA 16:0 & SFA \\
FA 18:0 & SFA \\
FA 16:1 & MUFA \\
FA 18:1 & MUFA \\
FA 18:2 & PUFA \\
FA 20:3 & PUFA \\
FA 20:4 & PUFA \\
FA 20:5 & PUFA \\
FA 22:5 & PUFA \\
FA 22:6 & PUFA \\
\hline
\end{tabular}




\section{Chapter 3}

Supplementary table 4: Clamp parameters in control participants, and patients with NAFL and T2D

\begin{tabular}{lccc}
\hline & Control $(n=7)$ & NAFL $(n=14)$ & T2D $(n=9)$ \\
\hline Ra $(\mu \mathrm{mol} / \mathrm{kg} / \mathrm{min})$ & & & \\
Baseline & $9.74 \pm 2.40$ & $7.64 \pm 1.50$ & $12.84 \pm 2.03^{\mathrm{A}, \mathrm{B}}$ \\
$\quad$ Low insulin & $11.88 \pm 1.34$ & $9.08 \pm 1.69^{\mathrm{A}}$ & $9.83 \pm 2.44$ \\
$\quad$ High insulin & $35.16 \pm 11.29$ & $26.58 \pm 7.74$ & $23.02 \pm 10.76$ \\
Rd ( $\mu \mathrm{mol} / \mathrm{kg} / \mathrm{min})$ & & & \\
Baseline & $8.87 \pm 2.06$ & $7.18 \pm 1.64$ & $13.28 \pm 3.10^{\mathrm{A}, \mathrm{B}}$ \\
Low insulin & $12.68 \pm 1.30$ & $9.04 \pm 1.58^{\mathrm{A}}$ & $10.28 \pm 2.47^{\mathrm{A}}$ \\
High insulin & $35.41 \pm 10.80$ & $26.16 \pm 7.51$ & $23.55 \pm 9.93^{\mathrm{A}}$ \\
EGP ( $\mu$ mol/kg/min) & & & \\
Baseline & $9.74 \pm 2.40$ & $7.64 \pm 1.50$ & $12.84 \pm 2.03^{\mathrm{A}, \mathrm{B}}$ \\
Low insulin & $2.68 \pm 2.55$ & $3.15 \pm 1.22$ & $7.54 \pm 2.09^{\mathrm{A}, \mathrm{B}}$ \\
High insulin & $0.98 \pm 1.47$ & $1.07 \pm 0.81$ & $2.73 \pm 0.51^{\mathrm{A}, \mathrm{B}}$ \\
\% suppression low insulin & $74.20 \pm 18.40$ & $56.92 \pm 18.50$ & $41.61 \pm 12.15^{\mathrm{A}}$ \\
\% suppression high insulin & $89.33 \pm 15.82$ & $85.24 \pm 12.06$ & $78.44 \pm 4.03$ \\
\hline
\end{tabular}

Data are presented as mean \pm SD. Overweight/obese controls without NAFL (controls, $n=7$ ), overweight/obese with NAFL (NAFL, $n=14$ ), patients with type 2 diabetes $(T 2 D, n=9)$. Ra baseline was significantly higher in $T 2 D$ compared to control $(p=0.010)$ and NAFL $\left(p=2.0 \times 10^{-6}\right)$. Ra low insulin was significantly lower in NAFL compared to control $(p=0.010)$. Rd baseline was significantly higher in T2D compared to control $(p=0.002)$ and NAFL $\left(p=3.0 \times 10^{-6}\right)$. Rd low insulin was significantly higher in control compared to NAFL $(p=0.001)$ and T2D $(p=0.045)$. Rd high insulin was significantly lower in T2D compared to control $(p=0.045)$. EGP baseline was significantly higher in T2D compared to control $(p=0.010)$ and NAFL $\left(p=2.0 \times 10^{-6}\right)$. EGP low insulin was significantly higher in T2D compared to control $\left(p=2.35 \times 10^{-4}\right)$ and NAFL $(p=0.001)$. EGP high insulin was significantly higher in T2D compared to control $(p=0.003)$ and NAFL $(p=0.001)$. EGP suppression low insulin was significantly lower in T2D compared to control $(p=0.002)$. A Significantly different from control, ${ }^{B}$ significantly different from NAFL (Kruskal-Wallis p $<0.05$ for EGP Low Insulin and EGP suppression high insulin, and one-way ANOVA $p<0.05$ for all other parameters). Bonferroni correction was used for post-hoc analyses. 


\section{REFERENCES}

1. Sayiner M, Koenig A, Henry L, Younossi ZM. Epidemiology of Nonalcoholic Fatty Liver Disease and Nonalcoholic Steatohepatitis in the United States and the Rest of the World. Clinics in liver disease. 2016;20(2):205-14.

2. Gaggini M, Morelli M, Buzzigoli E, DeFronzo RA, Bugianesi E, Gastaldelli A. Nonalcoholic fatty liver disease (NAFLD) and its connection with insulin resistance, dyslipidemia, atherosclerosis and coronary heart disease. Nutrients. 2013;5(5):1544-60.

3. Calzadilla Bertot L, Adams LA. The Natural Course of Non-Alcoholic Fatty Liver Disease. International journal of molecular sciences. 2016;17(5).

4. Mantovani A, Byrne CD, Bonora E, Targher G. Nonalcoholic Fatty Liver Disease and Risk of Incident Type 2 Diabetes: A Meta-analysis. Diabetes care. 2018;41(2):37282.

5. Targher G, Byrne CD, Lonardo A, Zoppini G, Barbui C. Non-alcoholic fatty liver disease and risk of incident cardiovascular disease: A meta-analysis. Journal of hepatology. 2016;65(3):589-600.

6. Lim S, Oh TJ, Koh KK. Mechanistic link between nonalcoholic fatty liver disease and cardiometabolic disorders. International journal of cardiology. 2015;201:408-14.

7. Targher G, Byrne CD. Clinical Review: Nonalcoholic fatty liver disease: a novel cardiometabolic risk factor for type 2 diabetes and its complications. The Journal of clinical endocrinology and metabolism. 2013;98(2):483-95.

8. Targher G, Day CP, Bonora E. Risk of cardiovascular disease in patients with nonalcoholic fatty liver disease. The New England journal of medicine. 2010;363(14):1341-50.

9. Brouwers B, Schrauwen-Hinderling VB, Jelenik T, Gemmink A, Havekes B, Bruls Y, et al. Metabolic disturbances of non-alcoholic fatty liver resemble the alterations typical for type 2 diabetes. Clinical science (London, England : 1979). 2017;131(15):1905-17.

10. Matikainen N, Adiels M, Söderlund S, Stennabb S, Ahola T, Hakkarainen A, et al. Hepatic lipogenesis and a marker of hepatic lipid oxidation, predict postprandial responses of triglyceride-rich lipoproteins. Obesity (Silver Spring, Md). 2014;22(8):1854-9.

11. Lambert JE, Ramos-Roman MA, Browning JD, Parks EJ. Increased de novo lipogenesis is a distinct characteristic of individuals with nonalcoholic fatty liver disease. Gastroenterology. 2014;146(3):726-35.

12. Gluchowski NL, Gabriel KR, Chitraju C, Bronson RT, Mejhert N, Boland S, et al. Hepatocyte Deletion of Triglyceride-Synthesis Enzyme Acyl CoA: Diacylglycerol Acyltransferase 2 Reduces Steatosis Without Increasing Inflammation or Fibrosis in Mice. Hepatology (Baltimore, Md). 2019.

13. Benhamed F, Denechaud PD, Lemoine M, Robichon C, Moldes M, Bertrand-Michel $J$, et al. The lipogenic transcription factor ChREBP dissociates hepatic steatosis from insulin resistance in mice and humans. The Journal of clinical investigation. 2012;122(6):2176-94. 
14. Puri P, Baillie RA, Wiest MM, Mirshahi F, Choudhury J, Cheung O, et al. A lipidomic analysis of nonalcoholic fatty liver disease. Hepatology (Baltimore, Md). 2007;46(4):1081-90.

15. Araya J, Rodrigo R, Videla LA, Thielemann L, Orellana M, Pettinelli $P$, et al. Increase in long-chain polyunsaturated fatty acid $n-6 / n-3$ ratio in relation to hepatic steatosis in patients with non-alcoholic fatty liver disease. Clinical science (London, England : 1979). 2004;106(6):635-43.

16. Johnson NA, Walton DW, Sachinwalla T, Thompson $\mathrm{CH}$, Smith $\mathrm{K}$, Ruell PA, et al. Noninvasive assessment of hepatic lipid composition: Advancing understanding and management of fatty liver disorders. Hepatology (Baltimore, Md). 2008;47(5):1513-23.

17. Hamilton G, Yokoo T, Bydder M, Cruite I, Schroeder ME, Sirlin CB, et al. In vivo characterization of the liver fat (1)H MR spectrum. NMR in biomedicine. 2011;24(7):784-90.

18. Lundbom J, Hakkarainen A, Söderlund S, Westerbacka J, Lundbom N, Taskinen MR. Long-TE $1 \mathrm{H}$ MRS suggests that liver fat is more saturated than subcutaneous and visceral fat. NMR in biomedicine. 2011;24(3):238-45.

19. Gajdosik M, Chadzynski GL, Hangel G, Mlynarik V, Chmelik M, Valkovic L, et al. Ultrashort-TE stimulated echo acquisition mode (STEAM) improves the quantification of lipids and fatty acid chain unsaturation in the human liver at $7 \mathrm{~T}$. NMR in biomedicine. 2015;28(10):1283-93.

20. Frahm J, Bruhn H, Gyngell ML, Merboldt KD, Hanicke W, Sauter R. Localized highresolution proton NMR spectroscopy using stimulated echoes: initial applications to human brain in vivo. Magnetic resonance in medicine. 1989;9(1):79-93.

21. Tkac I, Starcuk Z, Choi IY, Gruetter R. In vivo 1 H NMR spectroscopy of rat brain at 1 ms echo time. Magnetic resonance in medicine. 1999;41(4):649-56.

22. Dong Z, Dreher W, Leibfritz D. Experimental method to eliminate frequency modulation sidebands in localized in vivo $1 \mathrm{H}$ MR spectra acquired without water suppression. Magnetic resonance in medicine. 2004;51(3):602-6.

23. Diraison F, Pachiaudi C, Beylot M. In vivo measurement of plasma cholesterol and fatty acid synthesis with deuterated water: determination of the average number of deuterium atoms incorporated. Metabolism: clinical and experimental. 1996;45(7):817-21.

24. Dempster P, Aitkens S. A new air displacement method for the determination of human body composition. Medicine and science in sports and exercise. 1995;27(12):1692-7.

25. Siri WE. Body composition from fluid spaces and density: analysis of methods. 1961. Nutrition (Burbank, Los Angeles County, Calif). 1993;9(5):480-91; discussion , 92.

26. Lofgren L, Stahlman M, Forsberg GB, Saarinen S, Nilsson R, Hansson GI. The BUME method: a novel automated chloroform-free 96-well total lipid extraction method for blood plasma. J Lipid Res. 2012. 
27. Lofgren L, Forsberg GB, Stahlman M. The BUME method: a new rapid and simple chloroform-free method for total lipid extraction of animal tissue. Scientific reports. 2016;6:27688.

28. Murphy RC, James PF, McAnoy AM, Krank J, Duchoslav E, Barkley RM. Detection of the abundance of diacylglycerol and triacylglycerol molecular species in cells using neutral loss mass spectrometry. Analytical biochemistry. 2007;366(1):59-70.

29. Steele R. Influences of glucose loading and of injected insulin on hepatic glucose output. Annals of the New York Academy of Sciences. 1959;82:420-30.

30. Donnelly KL, Smith Cl, Schwarzenberg SJ, Jessurun J, Boldt MD, Parks EJ. Sources of fatty acids stored in liver and secreted via lipoproteins in patients with nonalcoholic fatty liver disease. The Journal of clinical investigation. 2005;115(5):1343-51.

31. Bandsma RH, Prinsen BH, van Der Velden Mde S, Rake JP, Boer T, Smit GP, et al. Increased de novo lipogenesis and delayed conversion of large VLDL into intermediate density lipoprotein particles contribute to hyperlipidemia in glycogen storage disease type 1a. Pediatric research. 2008;63(6):702-7.

32. Hudgins LC, Parker TS, Levine DM, Hellerstein MK. A dual sugar challenge test for lipogenic sensitivity to dietary fructose. The Journal of clinical endocrinology and metabolism. 2011;96(3):861-8.

33. Peter A, Cegan A, Wagner S, Elcnerova M, Konigsrainer A, Konigsrainer I, et al. Relationships between hepatic stearoyl-CoA desaturase-1 activity and mRNA expression with liver fat content in humans. American journal of physiology Endocrinology and metabolism. 2011;300(2):E321-6.

34. Timlin MT, Barrows BR, Parks EJ. Increased dietary substrate delivery alters hepatic fatty acid recycling in healthy men. Diabetes. 2005;54(9):2694-701.

35. Hodson L. Hepatic fatty acid synthesis and partitioning: the effect of metabolic and nutritional state. The Proceedings of the Nutrition Society. 2019;78(1):126-34.

36. Kim CW, Addy C, Kusunoki J, Anderson NN, Deja S, Fu X, et al. Acetyl CoA Carboxylase Inhibition Reduces Hepatic Steatosis but Elevates Plasma Triglycerides in Mice and Humans: A Bedside to Bench Investigation. Cell metabolism. 2017;26(2):394-406.e6.

37. Loomba R, Kayali Z, Noureddin M, Ruane P, Lawitz EJ, Bennett M, et al. GS-0976 Reduces Hepatic Steatosis and Fibrosis Markers in Patients With Nonalcoholic Fatty Liver Disease. Gastroenterology. 2018;155(5):1463-73.e6.

38. Lawitz EJ, Coste A, Poordad F, Alkhouri N, Loo N, McColgan BJ, et al. Acetyl-CoA Carboxylase Inhibitor GS-0976 for 12 Weeks Reduces Hepatic De Novo Lipogenesis and Steatosis in Patients With Nonalcoholic Steatohepatitis. Clinical gastroenterology and hepatology : the official clinical practice journal of the American Gastroenterological Association. 2018;16(12):1983-91.e3. 


\section{CHAPTER 4}

Hepatic glycogen is not modulated by one night of prolonged fasting in people with non-alcoholic fatty liver

Kay H.M. Roumans ${ }^{1}$, Pandichelvam Veeraiah ${ }^{1,2}$, Julian V Cven $/ 9 m p$

Bas Havekes ${ }^{3}$, Harry P.F. Peters ${ }^{4}$, Lucas Lindeboom 1,2 Dath SSch Auwen ${ }^{1}$,

Vera B. Schrauwen-Hinderling ${ }^{1,2}$.

6200 MD, Maastricht, The Netherland

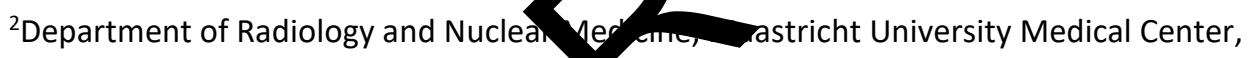

P.O. BOX 5800, 6202 AZ, Maastricht, The therlands.

${ }^{3}$ Department of Internal Medici \& Dy Ondocrinology and Metabolic Disease, Maastricht University Marical Ce M, P.O. BOX 5800, 6202 AZ, Maastricht, The Netherlands.

${ }^{4}$ Unilever Food now on Ce ter, Plantage 14, 6708 WJ Wageningen, The Netherlands.

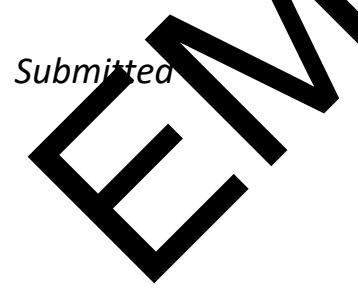




\section{CHAPTER 5}

The effect of a low versus high glycemic index and saturated fat diet on liver fat content and substrate metabolism

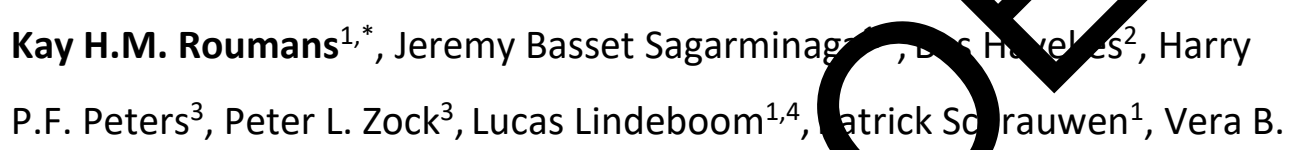
Schrauwen-Hinderling ${ }^{1,4}$.

${ }^{1}$ Department of Nutrition and Movem

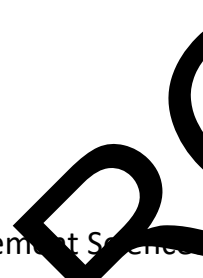
( 8 政 6200 MD, Maastricht, The Netbarlands.

${ }^{2}$ Department of Internal Medici , Dly ondocrinology and Metabolic Disease, Maastricht University Morical Ce A, P.O. BOX 5800, 6202 AZ, Maastricht, The Netherlands.

${ }^{3}$ Unilever Food Iprova Cente Plantage 14, 6708 WJ Wageningen, The Netherlands. ${ }^{4}$ Department of $\mathrm{R}$ a. Nogh Nuclear Medicine, Maastricht University Medical Center, P.O. BOX 020 A 6 , Maastricht, The Netherlands.

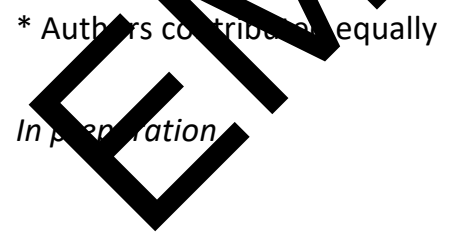




\section{CHAPTER 6}

Hepatic water and lipid T2 relaxation times are associated with level of steatosis - implications for absolute fat quantification

Pandichelvam Veeraiah ${ }^{1}$, Kay H.M. Roumans ${ }^{2}$, N MKe
C.G.J. Brouwers ${ }^{3}$, Yvonne M.H Bruls ${ }^{1}$, Matthijs K. Hesseli $k^{2}$, Joachim E. Wildberger ${ }^{1}$, Patrick Schrauwen ${ }^{2}$, Lucas , deb $^{-1,}$,

Vera B. Schrauwen-Hinderling. ${ }^{1,2}$
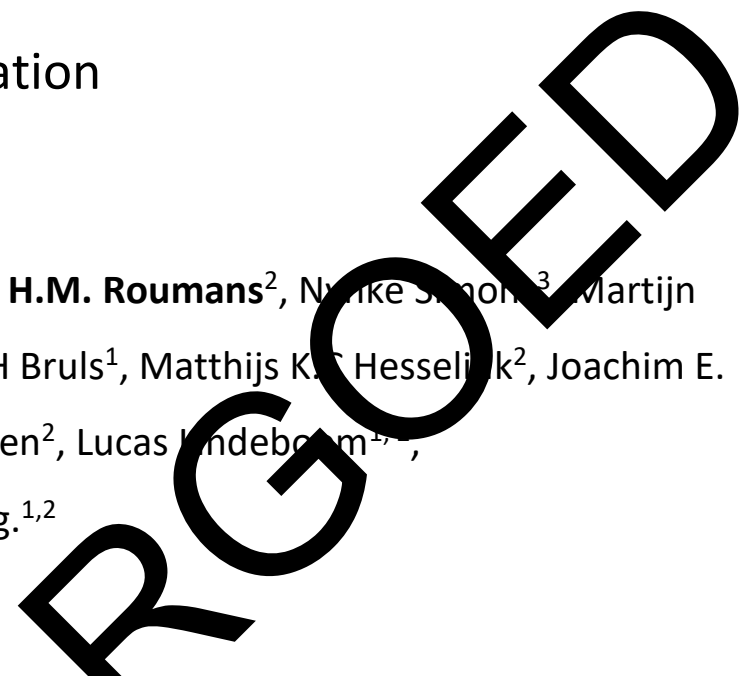

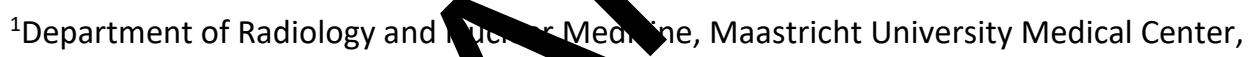
Maastricht, The Netherlands

${ }^{2}$ Department of Nutrit nat Mont Ment Sciences, Maastricht University Medical Center, Maastricht, The Neth lap

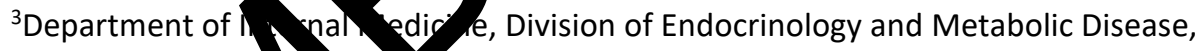
Maastricht/Univers v edical Center, Maastricht, The Netherlands 


\section{CHAPTER 7}

\section{Nicotinamide riboside supplementation alters body composition and skeletal muscle acetylcarnitine} concentrations in healthy obese humans

Carlijn M.E. Remie ${ }^{1}$, Kay H.M. Roumans ${ }^{1}$, Michiel P.B. Moonen ${ }^{1}$, Niels J. Connell ${ }^{1}$, Bas Havekes ${ }^{1,2}$, Julian Mevenkamp ${ }^{1,3}$, Lucas Lindeboom ${ }^{1,3}$, Vera H.W. de Wit ${ }^{1}$, Tineke van de Weijer ${ }^{1,3}$, Suzanne A.B.M. Aarts ${ }^{4}$, Esther Lutgens $^{4,5}$, Bauke V. Schomakers ${ }^{6,7}$, Hyung L. Elfrink ${ }^{6,7}$, Rubén ZapataPérez ${ }^{6}$, Riekelt H. Houtkooper ${ }^{6}$, Johan Auwerx ${ }^{8}$, Joris Hoeks ${ }^{1}$, Vera B. Schrauwen-Hinderling ${ }^{1,3}$, Esther Phielix ${ }^{1}$, Patrick Schrauwen ${ }^{1}$

\footnotetext{
${ }^{1}$ Department of Nutrition and Movement Sciences, School for Nutrition and Translational Research in Metabolism, Maastricht University, Maastricht, Netherlands.

${ }^{2}$ Division of Endocrinology, Department of Internal Medicine, Maastricht University Medical Center, Maastricht, Netherlands.

${ }^{3}$ Department of Radiology and Nuclear Medicine, Maastricht University Medical Center, Maastricht, Netherlands.

${ }^{4}$ Department of Medical Biochemistry, Amsterdam Cardiovascular Sciences, Amsterdam University Medical Centers, University of Amsterdam, Amsterdam, Netherlands.

${ }^{5}$ Institute for Cardiovascular Prevention (IPEK), Ludwig Maximilian University, Munich, Germany.

${ }^{6}$ Laboratory Genetic Metabolic Diseases, Amsterdam Gastroenterology and Metabolism, Amsterdam Cardiovascular Sciences, Amsterdam University Medical Centers, University of Amsterdam, Amsterdam, Netherlands.

${ }^{7}$ Core Facility Metabolomics, Amsterdam University Medical Centers, University of Amsterdam, Amsterdam, Netherlands.

${ }^{8}$ Laboratory of Integrative and Systems Physiology, École Polytechnique Fédérale de Lausanne, Lausanne, Switzerland.
}

Am J Clin Nutr 2020;00:1-14 


\section{ABSTRACT}

Background: Nicotinamide riboside (NR) is a $\mathrm{NAD}^{+}$precursor that boosts cellular $\mathrm{NAD}^{+}$ concentrations. Preclinical studies have shown profound metabolic health effects after NR supplementation.

Objective: Investigate the effects of six weeks NR supplementation on insulin sensitivity, mitochondrial function and other metabolic health parameters in overweight and obese volunteers.

Design: A randomized, double-blinded, placebo-controlled, cross-over intervention study was conducted in thirteen healthy overweight and obese men and women. Participants received six weeks NR (1000 $\mathrm{mg} /$ day) and placebo supplementation, followed by broad metabolic phenotyping, including hyperinsulinemic-euglycemic clamps, magnetic resonance spectroscopy, muscle biopsies, and assessment of ex vivo mitochondrial function and in vivo energy metabolism.

Results: Markers of increased NAD ${ }^{+}$synthesis - NAAD and MeNAM - were elevated in skeletal muscle after NR compared to placebo. NR increased body fat free mass $(62.65 \pm$ 2.49 vs. $61.32 \pm 2.58,1.34 \pm 0.50 \%$ in NR, placebo, and the change respectively, $p=0.02$ ) and increased sleeping metabolic rate. Interestingly, acetylcarnitine concentrations in skeletal muscle were increased upon NR (4558 \pm 749 vs. $3025 \pm 316$ and $1533 \pm 683$ $\mathrm{pmol} / \mathrm{mg}$ dry weight in NR, placebo, and the change respectively, $\mathrm{p}=0.04$ ) and the capacity to form acetylcarnitine upon exercise was higher in NR compared to placebo $(2.99 \pm 0.30$ vs. $2.40 \pm 0.33$ and $0.53 \pm 0.21 \mathrm{mmol} / \mathrm{kg}$ wet weight in $\mathrm{NR}$, placebo, and the change respectively, $p=0.01$ ). However, no effects of $N R$ were found on insulin sensitivity, mitochondrial function, hepatic and intramyocellular lipid accumulation, cardiac energy status, cardiac ejection fraction, ambulatory blood pressure, plasma markers of inflammation or energy metabolism.

Conclusion: NR supplementation of $1000 \mathrm{mg} /$ day for six weeks in healthy overweight and obese men and women increased skeletal muscle $\mathrm{NAD}^{+}$metabolites, affected skeletal muscle acetylcarnitine metabolism, and induced minor changes in body composition and sleeping metabolic rate. However, no other metabolic health effects were observed. 


\section{INTRODUCTION}

Nicotinamide riboside (NR) is a naturally occurring vitamin B3 present in the human diet, acts as a $N A D^{+}$precursor (1), and is suggested to improve mitochondrial function and insulin sensitivity (2). NR acts via activation of the $N A D^{+}$-dependent sirtuin enzyme family, thereby regulating oxidative metabolism (3-7). In vitro experiments have shown the successful NAD ${ }^{+}$ restoring capability of NR supplementation and the subsequent increase oxidative gene expression in skeletal muscle cells $(8,9)$. Results from in vivo mouse models have shown improvements in insulin sensitivity and oxidative energy metabolism, including enhanced metabolic flexibility, increased aerobic exercise capacity and indications of improved mitochondrial biogenesis (9-13). In these mouse models NR seems to specifically act on muscle, liver, heart and brown adipose tissue $(9,14)$. Reports indicate that $N A D^{+}$ metabolism including $\mathrm{NAD}^{+}$concentrations are decreased in the obese and older population (15-17). The number of human interventions with NR is small and the evidence that NR may have beneficial effects in humans is limited. Human pharmacokinetic studies showed increased circulatory $\mathrm{NAD}^{+}$metabolite concentrations in whole blood, PBMC and urine, after various dosages of NR supplementation (18-23), varying from 300 to $2000 \mathrm{mg} /$ day, indicating that NR supplementation is able to increase the $\mathrm{NAD}^{+}$pool in humans. So far, three randomized placebo-controlled NR supplementation studies have been performed in humans, in which the effect of NR supplementation on human metabolic health was investigated. Dollerup et al. $(21,24)$, investigated the effect of 2000 mg/day NR for 12 weeks in insulin resistant middle-aged obese men. No effects of NR supplementation were found on insulin sensitivity, muscle mitochondrial function or metabolic flexibility $(21,24)$. In addition, Martens et al. (20), investigated the effect of $1000 \mathrm{mg} /$ day NR for six weeks in healthy normal weight middle-aged and older men and women. Besides a trend in a reduction in a reduced arterial stiffness and lower blood pressure after NR supplementation, no effects were found on a wide variety of outcomes indicative of metabolic function, glucose metabolism, motor function and exercise capacity (20). Furthermore, Elhassan et al. (25) investigated the effects of $1000 \mathrm{mg} /$ day NR for 3 weeks in older men and showed increased skeletal muscle $\mathrm{NAD}^{+}$metabolite concentrations, however again no effect on skeletal muscle mitochondrial function was observed. To date, the effect of NR supplementation on both insulin sensitivity and skeletal muscle mitochondrial function has only been recently published in one human clinial trial $(21,24)$. Based on the promising preclinical findings reported until 2017, we designed a double blinded randomized placebo controlled cross-over study in which we aimed to investigate the effect of $1000 \mathrm{mg} /$ day NR supplementation on metabolic health in healthy obese and overweight men and women. The primary focus was on insulin sensitivity and muscle mitochondrial function, and secondary outcomes were related to energy metabolism and skeletal muscle 
$\mathrm{NAD}^{+}$metabolites. Therefore, our study expands the limited knowledge of the effect of NR supplementation on human metabolic health and aims to investigate the translational value of previous promising preclinical findings.

\section{METHODS}

The study was conducted in accordance with the principles of the declaration of Helsinki and approved by the Ethics Committee of the Maastricht University Medical Center. The study was registered at https://clinicaltrials.gov (NCT02835664). All participants provided written informed consent before screening.

\section{Participants}

Recruitment and data collection took place between December 2016 and December 2018 in Maastricht, The Netherlands. Thirteen participants completed the study, two dropped out because of personal or medical reasons (see Supplementary figure 1). Men and postmenopausal women were included. Participants underwent a screening including assessment of blood biochemistry, electrocardiography, anthropometric measurements and a questionnaire (including Baecke physical activity questionnaire (26)) to evaluate eligibility. Inclusion criteria were $45-65$ years of age, BMI $27-35 \mathrm{~kg} / \mathrm{m}^{2}$, sedentary lifestyle ( $<3 \mathrm{~h}$ exercise per week), non-smoking for at least 6 months, alcohol use of $\leq 2$ servings per day, stable body weight for at least 6 months and having no active diseases.

\section{Study design}

The study had a randomized controlled, double blinded, placebo controlled, cross-over design. Simple randomization was applied with use of computer-generated random numbers. Each participant received two interventions each of which lasted six weeks. During the first six weeks the participant was randomized to receive either NR supplementation or placebo. This six-week period was followed by a washout period of four to seven weeks. At the end of the washout period the participant crossed over to the treatment they did not receive during the first six weeks. In both intervention arms participants underwent exactly the same tests to determine the effect of the intervention (figure 1). These tests were performed in the end of week five and during week six. During the entire study period no changes in lifestyle (no change in diet, physical activity level or medication or supplement use) were allowed. Compliance was checked on a weekly basis by pill count. Furthermore, a fasted blood sample was taken, and body weight was monitored. During supplementation periods, any adverse effects and side effects were noted. 


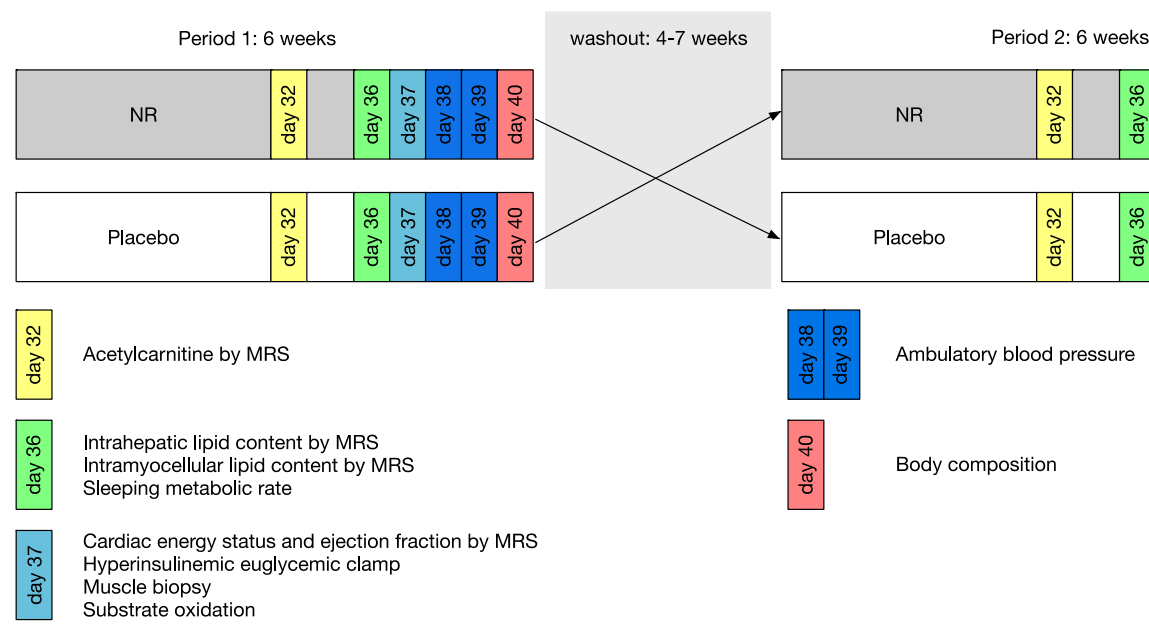

Figure 1: Study design. In this cross-over study, participants were randomly assigned to start with six weeks of NR supplementation or six weeks of placebo treatment. After a washout period of four-to-seven weeks, participants entered the other intervention arm such that all participants served as their own control. Participants were studied in week five (day 32) and in week six (day $36-40$ ) of each of the two interventions.

\section{Determination of sleeping metabolic rate}

At day 36 of each intervention, participants received a standardized dinner at 18:30 h after which they remained fasted and entered a metabolic chamber at 19:30 $\mathrm{h}$. The metabolic chamber is a small room with a bed, toilet, television, computer and access to water in which oxygen consumption and carbohydrate production were measured continuously in sampled room air. During the overnight stay in the respiration chamber sleeping metabolic rate was assessed. Participants decided themselves what time they went to sleep. At 06:00 $h$ the next morning participants were woken up and left the chamber in an overnight fasted state.

\section{Hyperinsulinemic euglycemic clamp}

To determine insulin sensitivity, a two-step hyperinsulinemic euglycemic clamp with coinfusion of D-[6.6- $\left.{ }^{2} \mathrm{H}_{2}\right]$ glucose tracer $(0.04 \mathrm{mg} / \mathrm{kg} / \mathrm{min})$ was performed on day 37 of both interventions (27). Insulin was infused, starting at $10: 30 \mathrm{~h}$, at $10 \mathrm{mU} / \mathrm{m}^{2} / \mathrm{min}$ for $2.5 \mathrm{~h}$ to assess hepatic insulin sensitivity and subsequently increased to $40 \mathrm{mU} / \mathrm{m}^{2} / \mathrm{min}$ for $2 \mathrm{~h}$ to measure whole body insulin sensitivity. Blood was frequently sampled to measure glucose concentrations directly from arterialized blood and tracer-enriched $20 \%$ glucose was coinfused at a variable rate to maintain euglycemia ( $5.0 \mathrm{mmol} / \mathrm{l})$ and reach steady state condition. During the steady state of 30 minutes, blood samples were collected, and 
substrate utilization was measured using indirect calorimetry. Due to technical failures, one participant was excluded from the clamp analysis.

\section{Skeletal muscle biopsies}

On day 37 of each intervention, a muscle biopsy was taken at 8:30 h after an overnight stay in the respiration chamber from the $\mathrm{m}$. vastus lateralis under local anesthesia (1\% lidocaine, without epinephrine) using the Bergström technique (28). The muscle biopsy was divided in several parts. One part was immediately frozen in melting isopentane for biochemical analyses. The remaining part was used for mitochondrial respiration analysis.

\section{Skeletal muscle $\mathrm{NAD}^{+}$metabolites}

$\mathrm{NAD}^{+}$content was determined in muscle biopsies using an enzymatic spectrophotometric cycling assay based on the coupled reaction of malate and alcohol dehydrogenases, as previously described (29). $\mathrm{NAD}^{+}$metabolites were measured in muscle biopsies through metabolomics. Freeze-dried muscle tissue (2-4 mg) was transferred to $2 \mathrm{~mL}$ tubes, and then $425 \mu \mathrm{L}$ water, $500 \mu \mathrm{L}$ methanol and $75 \mu \mathrm{L}$ of internal standards mixture (see Supplementary table 1) was added to each sample. Samples were homogenized using Tissuelyser II (Qiagen; $5 \mathrm{~min}$ at 30/s), followed by addition of $1000 \mu \mathrm{L}$ chloroform and thorough mixing. After centrifugation, the top layer containing the polar phase was transferred to $1.5 \mathrm{~mL}$ tubes and dried in a vacuum evaporator at $60^{\circ} \mathrm{C}$. Dried samples were reconstituted in 100 $\mu \mathrm{L}$ methanol/water $(6 / 4, \mathrm{v} / \mathrm{v})$ and analyzed in an Aquity UPLC system (Waters) coupled to an Impact IITM Ultra-High Resolution qTOF mass spectrometer (Bruker). Chromatographic separation of the compounds was achieved using a SeQuant ZIC-cHILIC column (PEEK $100 \mathrm{x}$ $2.1 \mathrm{~mm}, 3 \mu \mathrm{m}$ particle size, Merck, Darmstadt, Germany) at $30^{\circ} \mathrm{C}$. The LC method consisted in a gradient running at $0.25 \mathrm{~mL} / \mathrm{min}$ from $100 \%$ mobile phase $B$ (9:1 acetonitrile:water with $5 \mathrm{mM}$ ammonium acetate $\mathrm{pH} 6.8$ ) to $100 \%$ mobile phase A (1:9 acetonitrile:water with 5 $\mathrm{mM}$ ammonium acetate $\mathrm{pH} 6.8$ ) in 28 minutes, followed by a re-equilibration step at $100 \%$ $B$ of 5 minutes. MS data were acquired both in negative and positive ionization modes in full scan mode over the range of $\mathrm{m} / \mathrm{z}$ 50-1200. Due to limited sample availability, $\mathrm{NAD}^{+}$ content was measured in muscle biopsies of 8 participants and $\mathrm{NAD}^{+}$metabolomics was measured in muscle biopsies of 12 participants. 


\section{Skeletal muscle mitochondrial respiration and protein content}

From the muscle biopsies, permeabilized muscle fibers were prepared as described elsewhere (30). Thereafter ex vivo mitochondrial respiration was determined by measuring oxygen consumption rate upon addition of several substrates using high-resolution respirometry (Oxygraph, OROBOROS Instruments, Innsbruck, Austria) as described previously (31). All measurements were performed in quadruplicate and the integrity of the outer mitochondrial membrane was assessed in every experiment by the addition of cytochrome C (10 $\mu \mathrm{mol} / \mathrm{L})$ upon maximal coupled respiration. The average cytochrome $C$ response in the included traces was $1.7 \pm 0.03 \%$ and traces with a cytochrome $C$ response above $15 \%$ were excluded from statistical analyses. Data is presented per mg wet weight. Oxphos complex protein content was measured in skeletal muscle biopsies of 12 participants as previously described (32).

\section{Skeletal muscle acylcarnitine concentrations}

2-4 $\mathrm{mg}$ of freeze-dried muscle tissue was homogenized $1 \mathrm{~mL} 80 \%$ acetonitrile containing 50 $\mu \mathrm{L}$ of the internal standards. After centrifugation at $16000 \mathrm{~g}$, the resulting supernatant was dried under a stream of nitrogen at $40{ }^{\circ} \mathrm{C}$ and derivatized by addition of 1 propanol/acetylchloride $(4 / 1, \mathrm{v} / \mathrm{v})$ during 15 minutes at $60{ }^{\circ} \mathrm{C}$. After evaporation under nitrogen at $40{ }^{\circ} \mathrm{C}$, samples were redissolved in pure acetonitrile. Determination of the propylated acylcarnitines in the medium was performed by mass spectrometry in an Acquity UPLC System (Waters) coupled to a Quattro Premier XE Tandem Quadrupole Mass Spectrometer (Waters).

\section{$\mathrm{VO}_{2}$ peak}

Maximal oxygen consumption ( $\mathrm{VO}_{2}$ peak) was assessed during an incremental cycling test on an ergometer (Lode Excalibur Sport, Groningen, The Netherlands) (33), in the first week of the first supplementation period. After a warming-up of five minutes at $75 \mathrm{Watt}$, the power was increased every 2.5 minutes by 50 Watt until $80 \%$ of the maximally calculated heart rate was reached, then the power was increased every 2.5 minutes by 25 Watt until exhaustion. The highest average oxygen consumption over 25 seconds was used as the $\mathrm{VO}_{2}$ peak, reflecting physical fitness of the participant. 


\section{MR spectroscopy}

Proton magnetic resonance spectroscopy $\left({ }^{1} \mathrm{H}-\mathrm{MRS}\right)$ was used to quantify intrahepatic lipid content $(\mathrm{IHL})$, intramuscular lipid content (IMCL) and skeletal muscle acetylcarnitine concentrations. Phosphorus magnetic resonance spectroscopy ( $\left.{ }^{31} \mathrm{P}-\mathrm{MRS}\right)$ was used for assessment of phosphocreatine (PCr) to adenosine triphosphate (ATP) ratio as a marker of the energy status and in vivo mitochondrial function of the heart muscle. Magnetic resonance imaging was used for determination of cardiac left ventricle ejection fraction (EF). All measurements were performed on a 3.0T whole body scanner (Achieva Tx, Philips Healthcare, Best, The Netherlands).

IHL \& IMCL: IHL quantification took place on day 36 of each intervention at 17:00 h. Participants were fasted for at least 3 hours. Spectra were acquired as described before (34). Values are given as $\mathrm{T} 2$ corrected ratios of the $\mathrm{CH}_{2}$ peak relative to the unsuppressed water peak, expressed as percentage. Consecutive, IMCL was measured in the $\mathrm{m}$. tibialis anterior of the left leg, as reported earlier (34). Values are given as T1- and T2-corrected ratios of the $\mathrm{CH}_{2}$ peak relative to the unsuppressed water peak, expressed as percentage. Due to analytical problems only 9 participants could be included in the analyses of IMCL.

Acetylcarnitine: Acetylcarnitine concentrations in skeletal muscle were acquired on day 32 of each intervention at 17:00 $\mathrm{h}$ in the evening. Participants were fasted for at least 3 hours and were asked to refrain from strenuous physical activity 48 hours prior to the measurement. Resting skeletal muscle Acetylcarnitine concentrations were measured using a T1-editing method, as described earlier (35). Additionally, Acetylcarnitine concentrations were measured after 30 minutes exercise (70\% maximal output on an ergometer). Acetylcarnitine values were converted to absolute concentrations as described earlier (36). The creatine peak was used as a reference.

Cardiac PCr/ATP ratio \& ejection fraction: PCr/ATP ratio was quantified by ${ }^{31} \mathrm{P}-\mathrm{MRS}$ on day 37 of each intervention at 06:30 h, using an ISIS sequence. Participants were positioned prone and headfirst in the MRI. A ${ }^{1} \mathrm{H}^{31} \mathrm{P}$ surface heart coil was placed beneath the participants chest. The voxel of interest was carefully placed around the left ventricle of the heart. Spectra were acquired during the end-systolic phase (NSA = 96, number of points = 2048 , bandwidth $=3000 \mathrm{~Hz}$ ) with a repetition time of 5-8 heartbeats, depending on heart rate. $\mathrm{PCr}$ and ATP resonances were quantified using a custom written MATLAB (MATLAB 2014b, The MathWorks, Inc., Natick, Massachusetts, United States) script and values were corrected for T1 saturation and expressed as ratio of PCr over gamma-ATP. Due to technical errors, only 11 participants are included in the analyses of PCr/ATP ratio. Left ventricular size was measured for 12 participants based of MRI images of the heart. Left ventricular EF 
was calculated from the end diastiolic volume (EDV) and end systolic volume (ESV) according to the Biplane Ellipsoid Model, as described earlier (37).

\section{Ambulatory blood pressure}

Ambulatory blood pressure (BP) was measured (Mobil-O-Graph, I.E.M., Stolberg, Germany) on day 38 and 39 of each intervention for two days and one night (36-hours). Mean systolic (SBP) and diastolic (DBP) blood pressure during day time and night time, and night time dipping were calculated as previously described (38). Night was defined as the mean time going to bed of all participants $(23: 38 \mathrm{~h})$ until the mean time waking up of all participants (8:05 h). Due to technical failures night time BP could only by obtained from 12 participants.

\section{Body composition}

Body composition was determined on day 40 of each intervention at 08:30 $\mathrm{h}$ after an overnight fast of at least 10 hours. Body mass and body volume were assessed using airdisplacement plethysmography using the BodPod device (Cosmed, Italy, Rome) according to the manufacturer's instructions (39) and previously reported (40).

\section{Blood sampling and analyses}

Glucose (Hk-CP, Axonlab, Amsterdam, The Netherlands) and free fatty acids (FFA) (NEFAHR, WAKO chemicals, Neuss, Germany) were analyzed enzymatically in EDTA plasma using a Pentra 400 (Horiba, Montpellier, France). Triglycerides (Sigma, Zwijndrecht, The Netherlands), cholesterol (CHOD-PAP, Roche Diagnostics, Mannheim, Germany) and HDL-C (CHOD-PAP, Roche Diagnostics, Mannheim,Germany) after precipitation of apoB-containing lipoproteins with phosphotungstic acid and magnesium ions, were analyzed in serum also using a Pentra 400. All samples from one participant were analyzed within one run. LDL-C was calculated for 12 participants according to the Friedewald equation (41). In a subset of 7 participants (age $60 \pm 3$ years; BMI $30.0 \pm 1.7 \mathrm{~kg} / \mathrm{m}^{2} ; \mathrm{n}=2$ women) inflammatory cytokine concentrations were measured on a Luminex ${ }^{\circledR}$ 200TM system using an inflammation 20plex human Procartaplex panel (eBioscience, EPX200-12185-901) containing markers for sESelectin; ICAM-1/CD54; IL-1 $\alpha$; IL-4; IL-12p70; IL-17A/CTLA-8; IP-10/CXCL10; MCP-1/CCL2; MIP-1 $\alpha / C C L 3 ;$ MIP-1 $\beta / C C L 4 ;$ sP-Selectin; TNF $\alpha$. 


\section{Calculations}

Energy expenditure was calculated based on the measured averaged oxygen and carbon dioxide concentrations in the inspired and expired gasses with the assumption that protein oxidation was negligible, using the Weir equation $(42,43)$. Sleeping metabolic rate was calculated as the lowest average 3h energy expenditure during the sleeping period. Glucose oxidation and fat oxidation rates were calculated according to Perronet et al. $(42,43)$. Steele's single pool non-steady state equations were used to calculate the rate of glucose appearance ( $\mathrm{Ra}$ ) and the rate of glucose disappearance ( $\mathrm{Rd}$ ) during the clamp (44). Volume of distribution was assumed to be $0.160 \mathrm{l} / \mathrm{kg}$ for glucose. The change in insulin-stimulated glucose disposal (delta $\mathrm{Rd}$ ) was calculated by the difference between Rd under insulinstimulated conditions and Rd under basal non-insulin-stimulated conditions. Endogenous glucose production (EGP) was calculated as Ra minus exogenous glucose infusion rate. Nonoxidative glucose disposal (NOGD) was calculated as Rd minus carbohydrate oxidation.

\section{Sample size}

The sample size was determined based on demonstrating the statistical superiority of NR on insulin stimulated skeletal muscle glucose disposal compared with placebo. Twelve participants were required to achieve $80 \%$ power with an alpha of $5 \%$, an assumed treatment difference of within person changes of $3.25 \mu \mathrm{mol} / \mathrm{kg} / \mathrm{min}$, and an assumed SD of within person changes of $3.60 \mu \mathrm{mol} / \mathrm{kg} / \mathrm{min}$ for a one-group paired t-test for a hyperinsulinemic euglycemic clamp. A dropout of $20 \%$ was taken into account, so 15 participants were recruited. The expected effect size and SD was based on previous research within our research group (45).

\section{Statistical analyses}

Data are reported as mean $\pm S E$, unless otherwise stated. Data are presented for $n=13$, unless otherwise indicated. Differences between interventions were analyzed with a twotailed paired Students t-test for parametric data and with a Wilcoxon test for nonparametric data. A two-tailed $\mathrm{p}<0.05$ was considered statistically significant. Statistical analyses were performed using IBM SPSS version 23.0 for MacOS. 


\section{RESULTS}

\section{Participant population and study compliance}

Thirteen healthy overweight and obese men and women (age $59 \pm 5$ years; BMI $30.2 \pm 2.6$ $\mathrm{kg} / \mathrm{m}^{2}$ [mean $\pm \mathrm{SD}$ ]; 7 women) participated in the study. Participants were non-smokers, had no active diseases, used no medication or supplements interfering with the study outcomes, had a sedentary lifestyle according to the Baecke questionnaire (7.51 \pm 1.16 arbitrary units [mean $\pm \mathrm{SD}$ ]) and an average $\mathrm{VO}_{2}$ peak of $27.0 \pm 5.7 \mathrm{ml} / \mathrm{min} / \mathrm{kg}$ [mean $\pm \mathrm{SD}$ ] (see Supplementary table 2). NR at $1000 \mathrm{mg} /$ day was well tolerated and no adverse events or side effects were reported. Surplus NR and placebo supplements were returned by the participants and compliance rate was calculated as the proportion of capsules ingested relative to the prescribed number. The mean compliance rate during the six-week NR period was $99.3 \pm 1.8 \%$ [mean \pm SD] and during the six-week placebo period was $99.1 \pm 2.2 \%$ [mean \pm SD]. Participants were instructed to maintain their habitual diet and physical activity pattern during the entire study, this was confirmed by a stable body weight of $85.2 \pm 3.8 \mathrm{~kg}$ [mean \pm SD] after six weeks of NR supplementation compared to $85.5 \pm 3.8 \mathrm{~kg}$ [mean $\pm \mathrm{SD}$ ] upon six weeks placebo $(p=0.55)$.

A

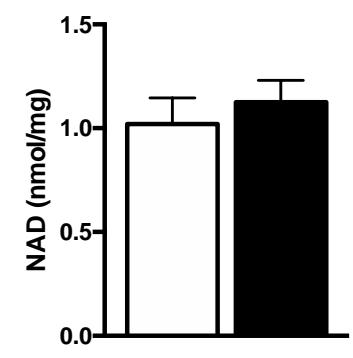

B

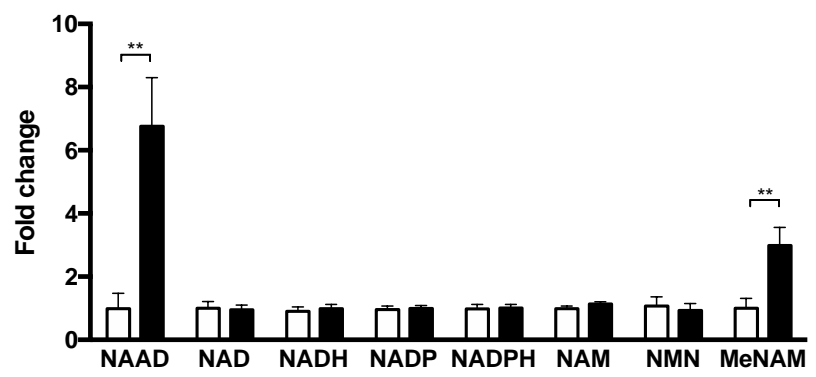

Figure 2: $\mathrm{NAD}^{+}$metabolites in skeletal muscle after NR and placebo supplementation. $A \mathrm{NAD}^{+}$concentrations measured in skeletal muscle biopsies by enzymatic assay, $n=8$. B NAD metabolites measured in skeletal muscle biopsies by mass spectrometry, $n=12$. Black bars is $N R$, open bars is placebo. Data are expressed as mean $\pm S E$. $P$ values are derived from the analysis of the mean within-person changes and the SEM of the within-group changes. ${ }^{* *} p<0.01$. 


\section{$\mathrm{NAD}^{+}$metabolites in skeletal muscle}

Compliance was further checked by analysis of $\mathrm{NAD}^{+}$-derived metabolites in muscle biopsy samples collected after six weeks of NR and placebo, 14-16 hours after supplement intake. First skeletal muscle $\mathrm{NAD}^{+}$content was measured. Quantitative analyses of $\mathrm{NAD}^{+}$ concentrations using enzymatic cycling assay in skeletal muscle showed that $\mathrm{NAD}^{+}$content was not different between NR and placebo (1.019 \pm 0.126 vs. $1.125 \pm 0.106,0.106 \pm 0.105$ $\mathrm{nmol} / \mathrm{mg}$ dry weight in NR, placebo, and the change respectively, $\mathrm{p}=0.34, \mathrm{n}=8$, figure $2 \mathrm{~A}$ ). The lack of increase in $\mathrm{NAD}^{+}$may indicate that $N A D^{+}$flux is increased without elevated steady state $\mathrm{NAD}^{+}$concentrations. Therefore, we next performed metabolomics to check if $\mathrm{NAD}^{+}$and $\mathrm{NAD}^{+}$metabolites in skeletal muscle were affected by NR. We confirmed that NR supplementation had no effect on skeletal muscle NAD ${ }^{+}$content itself $(0.96 \pm 0.15$ fold change in NR compared to placebo, $p=0.91, n=12$, figure $2 B$ ). However, oral $N R$ supplementation significantly increased two main markers of enhanced $\mathrm{NAD}^{+}$metabolism - nicotinic acid adenine dinucleotide (NAAD, $6.8 \pm 1.5$ fold change $p=<0.01, n=12$, Figure $2 B$ ) and methylnicotinamide (MeNAM, $3.0 \pm 0.6$ fold change $p=<0.01, n=12$, figure $2 B$ ), confirming that indeed NR was amplifying skeletal muscle $N A D^{+}$metabolism without affecting steady state. Moreover, NADH, NADP, NADPH, NAM and NMN concentrations remained unchanged $(p=0.73, p=0.79, p=0.75, p=0.25$ and $p=0.97$ respectively, $n=12$, see figure $2 \mathrm{~B}$ ).

\section{Mitochondrial respiration in skeletal muscle}

Supplementation with NR did not result in any change in mitochondrial respiration compared ot the placebo state. Respiration in the presence of substrate alone (state 2) (malate (M), malate + octanoyl carnitine (MO2) or malate + glutamate (MG2)) was not different between conditions $(p=0.34, p=0.19, p=0.74$ respectively, figure $3 A$ ). Furthermore, ADP stimulated (state 3 ) respiration on lipid-derived substrate (malate + octanoyl carnitine $+\operatorname{ADP}(\mathrm{MO3})$ ) and upon complex I substrates (malate + glutamate + ADP (MG3)) was unchanged ( $p=0.67$ and $p=0.64$ respectively, figure $3 B$ and $3 C$ ). Respiration upon parallel electron input to both complex I and II (malate + octanoyl carnitine + glutamate (MOG3)) was not different between conditions ( $49.87 \pm 2.80 \mathrm{vs}$. $50.92 \pm 2.44 \mathrm{pmol} / \mathrm{mg} / \mathrm{s},-1.04 \pm 2.58$ in NR, placebo, and the change respectively, $p=0.69$, figure 3D). Similar results were observed when succinate was sequentially added in both experiments (MGS3: $74.27 \pm 3.27$ vs. $72.90 \pm 4.64,1.37 \pm 5.01 \mathrm{pmol} / \mathrm{mg} / \mathrm{s}$ in NR, placebo, and the change respectively, $p=0.79$; MOGS3: $75.30 \pm 3.92$ vs. $76.09 \pm 3.31,-0.79 \pm 4.36 \mathrm{pmol} / \mathrm{mg} / \mathrm{s}$ in NR, placebo, and the change respectively, $p=0.86$, figure $3 \mathrm{D}$ ). Maximal $\mathrm{FCCP}$-induced uncoupled respiration (state $\mathrm{u})$, reflecting the maximal capacity of the electron transport chain, was also 
unchanged ( $p=0.81$, figure $3 E$ ). Finally, state 40 respiration (reflecting proton leak) was similar after NR and placebo conditions ( $p=0.89$, figure $3 F$ ). For a description of the different states, please see: https://www.bioblast.at/index.php/ MitoPedia: Respiratory states. Mitochondrial OXPHOS protein concentrations (Complex I $0.96 \pm 0.21$ vs. $0.91 \pm 0.15,0.05$ \pm 0.235 A.U. in NR, placebo, and the change respectively, $p=0.84$; Complex II $1.01 \pm 0.17$ vs. $0.78 \pm 0.05,0.23 \pm 0.20 \mathrm{~A}$.U. in NR, placebo, and the change respectively, $p=0.64$; Complex III $0.94 \pm 0.19$ vs. $0.92 \pm 0.17,0.02 \pm 0.22 \mathrm{~A}$.U. in NR, placebo, and the change respectively, $\mathrm{p}=0.93$; Complex IV $1.02 \pm 0.15$ vs. $0.94 \pm 0.10,0.09 \pm 0.14 \mathrm{~A}$.U. in NR, placebo, and the change respectively $p=0.55$; Complex V $1.00 \pm 0.17$ vs. $0.79 \pm 0.16,0.21 \pm 0.17$ A.U. in NR, placebo, and the change respectively $\mathrm{p}=0.24$ ) were similar after $N R$ and placebo supplementation, indicating no effect of NR on mitochondrial content (figure 3G). 
A

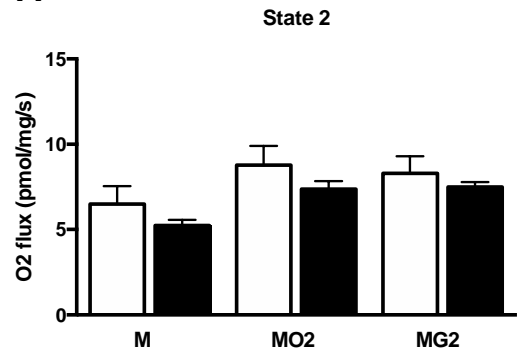

D

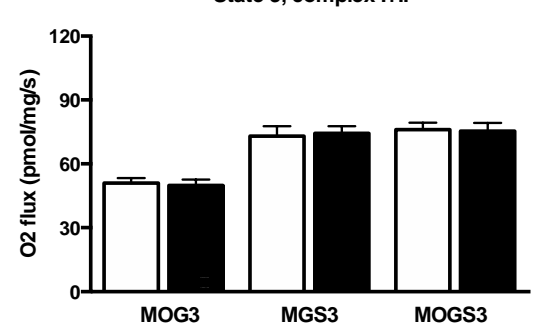

B

State 3, fat oxidation

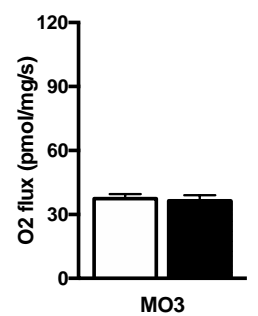

E State u, complex I+I

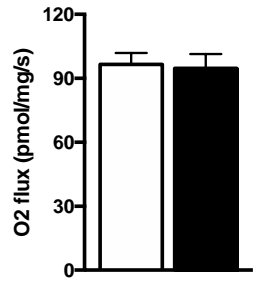

FCCP
C State 3, complex I

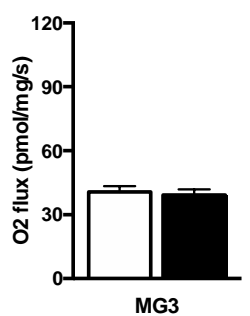

F State 40, complex I+II

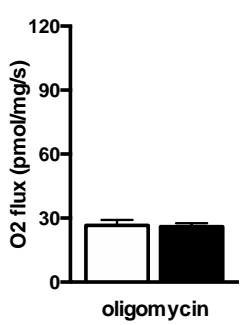

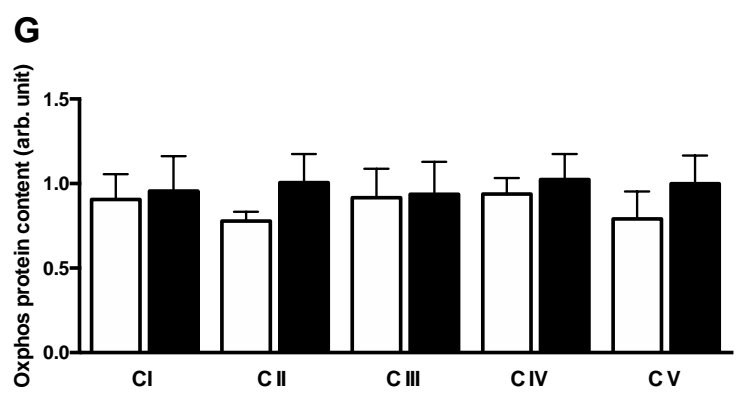

Figure 3: Skeletal muscle ex vivo mitochondrial respiratory capacity after NR and placebo supplementation. A State 2 respiration upon malate (M), malate + octanoyl carnitine (MO2) and malate + glutamate (MG2). B ADP stimulated state 3 respiration upon lipid derived substrate, malate + octanoyl carnitine + glutamate (MO3). C ADP stimulated state 3 respiration upon complex I substrates, malate + glutamate (MG3). D ADP stimulated state 3 respiration upon parallel electron input to both complex I and II, malate + octanoyl carnitine + glutamate (MOG3), malate + glutamate + succinate (MGS3), malate + octanoyl carnitine + glutamate + succinate (MOGS3). E State u: maximal FCCP-induced uncoupled respiration. F State 4o: Oligomycin induced respiration not coupled to ATP synthesis. G Protein content of individual complexes of the electron transport chain. Black bars is NR, open bars is placebo. $\mathrm{N}=12$. Data are expressed as mean $\pm \mathrm{SE}$. 


\section{Acetylcarnitine concentrations in skeletal muscle}

Quantification of acetylcarnitine concentration in skeletal muscle, measured by MRS technique in the early evening at 17:00 h after 3 hours fasting, revealed significantly lower baseline acetylcarnitine concentrations under NR supplementation compared to placebo $(1.30 \pm 0.16$ vs. $1.80 \pm 0.18,-0.53 \pm 0.19 \mathrm{mmol} / \mathrm{kg}$ wet weight in NR, placebo, and the change respectively, $p=0.02$, figure $4 \mathrm{~A})$. Maximally stimulated acetylcarnitine concentrations, measured upon exercise, were not different between conditions ( $4.29 \pm 0.29$ vs. $4.20 \pm 0.27$, $0.00 \pm 0.20 \mathrm{mmol} / \mathrm{kg}$ wet weight in NR, placebo, and the change respectively, $p=0.67$, figure $4 \mathrm{~A})$. Nonetheless, the capacity to increase acetylcarnitine formation, expressed as the change computed as the post exercise value minus the baseline value, was significantly higher in NR compared to placebo ( $2.99 \pm 0.30$ vs. $2.40 \pm 0.33,0.53 \pm 0.21 \mathrm{mmol} / \mathrm{kg}$ wet weight in NR, placebo, and the change respectively, $p=0.01$, figure $4 A$ ). Based on these results, we decided to perform full acylcarnitine analysis in skeletal muscle biopsies taken at 8:30 h after an overnight fast. Remarkably, acetylcarnitine (C2) concentrations were significantly higher under NR supplementation compared to placebo (C2: $4558 \pm 749$ vs. $3025 \pm 316,1533 \pm 683 \mathrm{pmol} / \mathrm{mg}$ dry weight in NR, placebo, and the change respectively, $p=0.04$; figure 4B). No differences were detected in free carnitine (CO), other short chain ( $\mathrm{C} 3$ to $\mathrm{C5}$ ), medium chain ( $\mathrm{C} 6$ to $\mathrm{C} 12$ ) or long chain acylcarnitines (C13 to $\mathrm{C20}$ ) between NR and placebo ( $p=0.25, p=0.27, p=0.99$ and $p=0.45$ respectively; figure $4 C$ ).

\section{Insulin sensitivity and substrate kinetics}

Potential effects of NR on whole body and tissue-specific insulin sensitivity were assessed by a two-step hyperinsulinemic-euglycemic clamp. Whole body insulin stimulated glucose uptake, as expressed by the change in glucose disposal (delta Rd) from baseline to high insulin dose, was not different between NR and placebo ( $p=0.98$, table 1$)$. Hepatic insulin sensitivity, reflected by EGP suppression (EGP\%) during the low insulin phase, was not affected by NR compared to placebo ( $p=0.30$, table 1 ). NR had no effect on baseline substrate oxidation (carbohydrate oxidation $p=0.84$, and fat oxidation $p=0.67$ ). Furthermore, insulin stimulated carbohydrate oxidation or suppression of fatty acid oxidation, reflecting metabolic flexibility, was not different between NR and placebo $(p=0.92$ and $p=0.70$ ) (see table 1 ). In addition, non-oxidative glucose disposal (delta NOGD) during low and high insulin phase of the clamp remained unchanged between conditions $(p=0.88$ and $p=0.99$ ) (see table 1). Plasma FFA concentrations were suppressed by insulin to a similar extend between NR and placebo $(p=0.97$ and $p=0.99$ during low and high insulin phase respectively, table 1 ), indicating similar white adipose tissue insulin sensitivity. 


\section{Chapter 7}

A

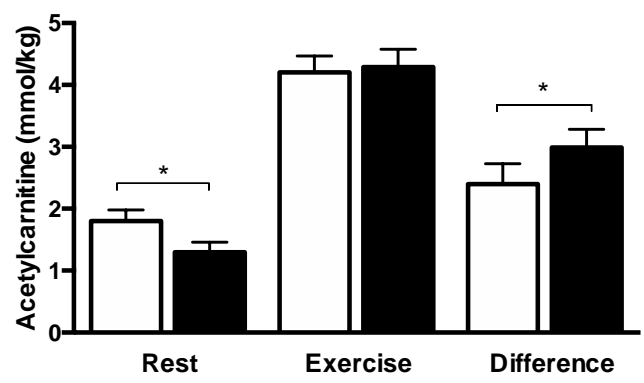

C

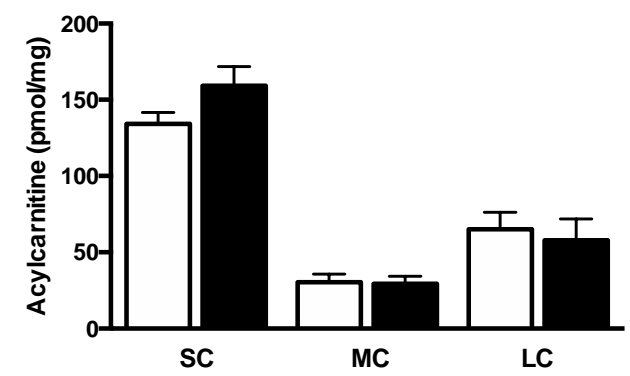

B

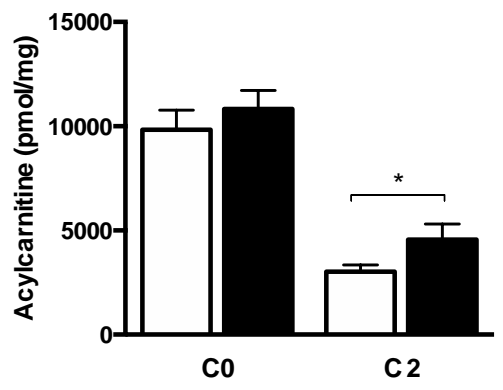

Figure 4: Skeletal muscle acylcarnitine concentrations measured in the morning and evening after NR and placebo supplementation. A Acetylcarnitine concentration measured by MRS in skeletal muscle in the evening during rest, after exercise and the capacity to form acetylcarnitine expressed as the difference between rest and exercise. B Free carnitine (CO) and acetylcarnitine (C2) concentrations measured in muscle biopsies in the morning during rest. C Sum of short chain acylcarnitines (SC), sum of medium chain acylcarnitines (MC) and sum of long chain acylcarnitines (LC) concentrations measured in biopsies during rest in the morning. Black bars is NR, open bars is placebo. $\mathrm{N}=13$. Data are expressed as mean $\pm \mathrm{SE}$. $\mathrm{P}$ values are derived from the analysis of the mean withinperson changes and the SEM of the within-group changes. ${ }^{*} p<0.05$. 
Table 1: Insulin sensitivity and substrate kinetics

\begin{tabular}{|c|c|c|c|c|}
\hline Parameter & Placebo & NR & Change & p-value \\
\hline \multicolumn{5}{|l|}{$\mathrm{Ra}(\mu \mathrm{mol} / \mathrm{kg} / \mathrm{min})^{1}$} \\
\hline Baseline & $9.32 \pm 0.33$ & $9.54 \pm 0.78$ & $0.22 \pm 0.72$ & 0.77 \\
\hline Low insulin & $11.66 \pm 0.93$ & $11.74 \pm 0.91$ & $0.08 \pm 0.42$ & 0.85 \\
\hline High insulin & $36.70 \pm 3.40$ & $37.60 \pm 2.66$ & $0.91 \pm 2.24$ & 0.69 \\
\hline \multicolumn{5}{|l|}{$\mathrm{Rd}(\mu \mathrm{mol} / \mathrm{kg} / \mathrm{min})^{1}$} \\
\hline Baseline & $9.90 \pm 0.53$ & $9.52 \pm 0.84$ & $-0.38 \pm 0.97$ & 0.70 \\
\hline Low insulin & $12.06 \pm 1.00$ & $12.36 \pm 0.94$ & $0.30 \pm 0.45$ & 0.51 \\
\hline High insulin & $36.76 \pm 3.36$ & $36.32 \pm 2.62$ & $-0.45 \pm 2.13$ & 0.84 \\
\hline Delta baseline-low insulin & $2.16 \pm 1.07$ & $2.84 \pm 1.01$ & $0.68 \pm 1.03$ & 0.47 \\
\hline Delta baseline-high insulin & $26.86 \pm 3.31$ & $26.79 \pm 2.86$ & $-0.07 \pm 2.17$ & 0.98 \\
\hline \multicolumn{5}{|l|}{$\mathrm{EGP}(\mu \mathrm{mol} / \mathrm{kg} / \mathrm{min})^{1}$} \\
\hline Baseline & $9.32 \pm 0.33$ & $9.54 \pm 0.78$ & $0.22 \pm 0.72$ & 0.77 \\
\hline Low insulin & $2.70 \pm 0.40$ & $3.38 \pm 0.56$ & $0.68 \pm 0.48$ & 0.18 \\
\hline$\%$ suppression low insulin & $70.44 \pm 4.57$ & $61.02 \pm 7.05$ & $-9.42 \pm 7.13$ & 0.30 \\
\hline High insulin & $-0.04 \pm 0.26$ & $0.51 \pm 0.56$ & $0.55 \pm 0.51$ & 0.30 \\
\hline$\%$ suppression high insulin & $99.78 \pm 2.52$ & $96.82 \pm 4.65$ & $-2.95 \pm 4.28$ & 0.50 \\
\hline \multicolumn{5}{|l|}{$\mathrm{NOGD}(\mu \mathrm{mol} / \mathrm{kg} / \mathrm{min})^{1}$} \\
\hline Baseline & $5.27 \pm 1.22$ & $4.88 \pm 1.06$ & $-0.40 \pm 1.18$ & 0.74 \\
\hline Low insulin & $3.77 \pm 0.92$ & $3.56 \pm 0.75$ & $-0.19 \pm 0.63$ & 0.76 \\
\hline High insulin & $21.28 \pm 2.84$ & $20.85 \pm 2.01$ & $-0.43 \pm 2.03$ & 0.84 \\
\hline Delta baseline-low insulin & $-1.51 \pm 1.36$ & $-1.30 \pm 0.85$ & $0.21 \pm 1.34$ & 0.88 \\
\hline Delta baseline-high insulin & $16.01 \pm 2.81$ & $15.97 \pm 2.04$ & $-0.03 \pm 1.83$ & 0.99 \\
\hline \multicolumn{5}{|c|}{ Carbohydrate oxidation ( $\mu \mathrm{mol} / \mathrm{kg} / \mathrm{min})$} \\
\hline Baseline & $4.42 \pm 0.81$ & $4.58 \pm 0.55$ & $0.16 \pm 0.61$ & 0.84 \\
\hline Low insulin & $7.84 \pm 0.69$ & $8.79 \pm 0.78$ & $0.95 \pm 0.76$ & 0.23 \\
\hline High insulin & $14.90 \pm 0.96$ & $14.99 \pm 1.15$ & $0.09 \pm 0.89$ & 0.92 \\
\hline \multicolumn{5}{|l|}{ Fat oxidation $(\mu \mathrm{mol} / \mathrm{kg} / \mathrm{min})$} \\
\hline Baseline & $3.78 \pm 0.23$ & $3.71 \pm 0.20$ & $-0.07 \pm 0.17$ & 0.67 \\
\hline Low insulin & $2.73 \pm 0.19$ & $2.55 \pm 0.17$ & $-0.18 \pm 0.21$ & 0.41 \\
\hline High insulin & $1.47 \pm 0.23$ & $1.38 \pm 0.22$ & $-0.09 \pm 0.22$ & 0.70 \\
\hline \multicolumn{5}{|l|}{ Plasma FFA ( $\mu \mathrm{mol} / \mathrm{l})$} \\
\hline Baseline & $555.34 \pm 30.87$ & $581.16 \pm 32.08$ & $25.83 \pm 35.55$ & 0.48 \\
\hline Low insulin & $128.55 \pm 21.74$ & $128.03 \pm 19.26$ & $-0.52 \pm 14.19$ & 0.97 \\
\hline High insulin & $49.84 \pm 8.26$ & $56.45 \pm 14.80$ & $6.61 \pm 10.04$ & 0.99 \\
\hline \multicolumn{5}{|l|}{ Respiratory exchange ratio } \\
\hline Baseline & $0.77 \pm 0.01$ & $0.77 \pm 0.01$ & $0.00 \pm 0.01$ & 0.83 \\
\hline Low insulin & $0.82 \pm 0.01$ & $0.83 \pm 0.01$ & $0.01 \pm 0.01$ & 0.37 \\
\hline High insulin & $0.91 \pm 0.01$ & $0.91 \pm 0.01$ & $0.00 \pm 0.01$ & 0.86 \\
\hline
\end{tabular}

$\mathrm{Ra}$, rate of appearance; Rd, rate of disappearance; EGP, endogenous glucose production; NOGD, non-oxidative glucose disposal; FFA, free fatty acids. ${ }^{1} n=12$. Data are expressed as mean \pm SE. $P$ values are derived from the analysis of the mean within-person changes and the SE of the within-group changes. 


\section{Plasma biochemistry and inflammatory markers}

NR supplementation did not affect fasting plasma glucose, triglycerides, total cholesterol, HDL-C, LDL-C, or inflammatory markers, including chemokine, cytokine or cell-adhesion molecule concentrations (table 2). However, interleukin 1 alpha (IL-1 $\alpha$ ) tended to be lower after NR supplementation compared to placebo (1.61 \pm 0.28 vs. $2.11 \pm 0.35$ respectively, $\mathrm{p}=0.06$, table 2).

Table 2: Blood biochemistry

\begin{tabular}{|c|c|c|c|c|}
\hline Parameter & Placebo & NR & Change & p-value \\
\hline Glucose (mmol/l) & $5.48 \pm 0.14$ & $5.44 \pm 0.13$ & $-0.04 \pm 0.10$ & 0.70 \\
\hline Triglycerides (mmol/l) & $1.57 \pm 0.35$ & $1.63 \pm 0.38$ & $0.06 \pm 0.08$ & 0.24 \\
\hline Total cholesterol (mmol/l) & $5.54 \pm 0.30$ & $5.55 \pm 0.35$ & $0.01 \pm 0.11$ & 0.99 \\
\hline HDL-C (mmol/l) & $1.32 \pm 0.12$ & $1.32 \pm 0.09$ & $-0.00 \pm 0.04$ & 0.99 \\
\hline LDL-C (mmol/l) ${ }^{1}$ & $3.42 \pm 0.18$ & $3.37 \pm 0.17$ & $-0.05 \pm 0.07$ & 0.52 \\
\hline sE-selectin $(\mathrm{pg} / \mathrm{ml})^{2}$ & $32726 \pm 5298$ & $34765 \pm 4354$ & $686 \pm 2068$ & 0.99 \\
\hline sP-selectin $(\mathrm{pg} / \mathrm{ml})^{2}$ & $21428 \pm 3662$ & $26645 \pm 4065$ & $2624 \pm 3739$ & 0.58 \\
\hline ICAM-1 (pg/ml) $)^{2}$ & $92493 \pm 19326$ & $129236 \pm 34547$ & $35350 \pm 27518$ & 0.58 \\
\hline TNF- $\alpha(\mathrm{pg} / \mathrm{ml})^{2}$ & $29.80 \pm 6.38$ & $31.85 \pm 6.14$ & $2.70 \pm 1.97$ & 0.22 \\
\hline $\mathrm{IL}-1 \alpha(\mathrm{pg} / \mathrm{ml})^{2}$ & $2.11 \pm 0.35$ & $1.61 \pm 0.28$ & $-0.57 \pm 0.24$ & 0.06 \\
\hline $\mathrm{IL}-4(\mathrm{pg} / \mathrm{ml})^{2}$ & $8.04 \pm 1.03$ & $8.86 \pm 1.41$ & $0.71 \pm 3.63$ & 0.69 \\
\hline IL-12p70 (pg/ml) $)^{2}$ & $76.07 \pm 2.73$ & $78.89 \pm 3.02$ & $1.94 \pm 2.82$ & 0.81 \\
\hline $\mathrm{IL}-17 \alpha(\mathrm{pg} / \mathrm{ml})^{2}$ & $6.36 \pm 1.36$ & $7.95 \pm 2.09$ & $1.76 \pm 1.02$ & 0.16 \\
\hline CXCL10 $(\mathrm{pg} / \mathrm{ml})^{2}$ & $3.71 \pm 0.55$ & $3.39 \pm 0.50$ & $-0.61 \pm 0.40$ & 0.22 \\
\hline CCL2 $(\mathrm{pg} / \mathrm{ml})^{2}$ & $127.39 \pm 24.54$ & $144.34 \pm 47.86$ & $21.42 \pm 40.12$ & 0.69 \\
\hline $\operatorname{CCL} 3(\mathrm{pg} / \mathrm{ml})^{2}$ & $15.42 \pm 8.59$ & $17.04 \pm 10.39$ & $2.43 \pm 1.92$ & 0.30 \\
\hline $\mathrm{CCL} 4(\mathrm{pg} / \mathrm{ml})^{2}$ & $23.89 \pm 5.98$ & $28.49 \pm 7.51$ & $4.52 \pm 4.45$ & 0.38 \\
\hline
\end{tabular}

Blood samples were taken in week six of NR supplementation and placebo after an overnight fast. ${ }^{1} n=12,{ }^{2} n=7$. Data are expressed as mean $\pm \mathrm{SE}$. $\mathrm{P}$ values are derived from the analysis of the mean within-person changes and the SE of the within-group changes.

\section{Body composition}

After six weeks of NR and placebo supplementation, several changes in body composition were detected. Percentage fat free mass (FFM) was significantly higher after NR compared to placebo $(62.65 \pm 2.49$ vs. $61.32 \pm 2.58,1.34 \pm 0.50 \%$ in NR, placebo, and the change respectively, $p=0.02$, figure $5 B$ ). In line with this, percentage fat mass (FM) was significantly lower after NR compared to placebo (37.35 \pm 2.49 vs. $38.68 \pm 2.58,-1.34 \pm 0.50 \%$ in NR, placebo, and the change respectively, $p=0.02$, figure $5 \mathrm{~B}$ ). However, total bodyweight remained unchanged $(p=0.55$, figure $5 A)$. 


\section{Sleeping metabolic rate}

Sleeping metabolic rate (SMR), measured during an overnight stay in a respiration chamber, was higher upon six weeks of NR compared to placebo (6.68 \pm 0.30 vs. $6.49 \pm 0.31,0.19 \pm$ $0.08 \mathrm{MJ} /$ day in NR, placebo, and the change respectively, $p=0.05$, figure $5 \mathrm{C}$ ). This could be explained by the increase in FFM and sleeping metabolic rate per FFM was not significantly different $(0.127 \pm 0.003$ vs. $0.125 \pm 0.003,0.00 \pm 0.00 \mathrm{MJ} / \mathrm{FFM} /$ day in NR, placebo, and the change respectively, $p=0.48$, figure $5 E$ ).

\section{Ectopic lipid storage}

Intrahepatic lipid content, measured by MRS, was not different between NR and placebo conditions ( $3.4 \pm 1.2$ vs. $3.4 \pm 1.3,0.0 \pm 0.0 \%$ in NR, placebo, and the change respectively, $\mathrm{p}=0.85$ ). Furthermore, intramyocellular lipid content, measured by MRS technique in the $\mathrm{m}$. tibialis anterior, was not affected by NR supplementation ( $0.5 \pm 0.1$ vs. $0.5 \pm 0.1,0.0 \pm 0.1 \%$ in NR, placebo, and the change respectively, $p=0.50$ ).

\section{Cardiac function}

To investigate if NR supplementation could affect cardiac energetics, we determined cardiac PCr/ATP ratios, which however were not affected by $N R(p=0.90$, table 3$)$. No differences were observed in left ventricular end systolic volume (ESV), end diastolic volume (EDV), stroke volume (SV) and subsequently ejection fraction (EF) between NR and placebo $(p=0.23, p=0.72, p=0.69$ and $p=0.24$ respectively, table 3$)$. NR supplementation had no effect on 24-hour systolic blood pressure (SBP), diastolic blood pressure (DBP), mean arterial pressure (MAP), pulse pressure (PP), and heart rate (HR) $(p=0.56, p=0.39, p=0.40$, $p=0.60$ and $p=0.60$ respectively, table 3 ). Separate analyses of day time and night time measurements did not reveal an effect of NR supplementation (see table 3 ). In addition, night time dipping of SBP and DBP was not affected by NR compared to placebo ( $p=0.53$ and $p=0.26$ respectively, table 3 ). 


\section{A}

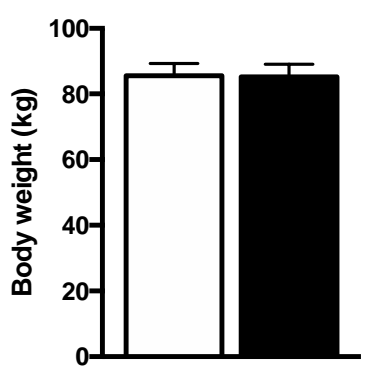

C

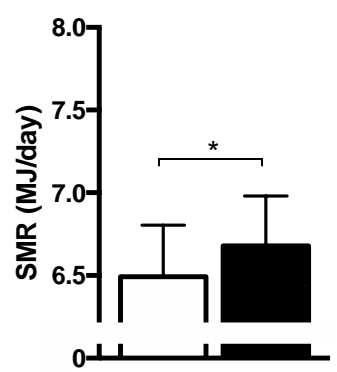

B

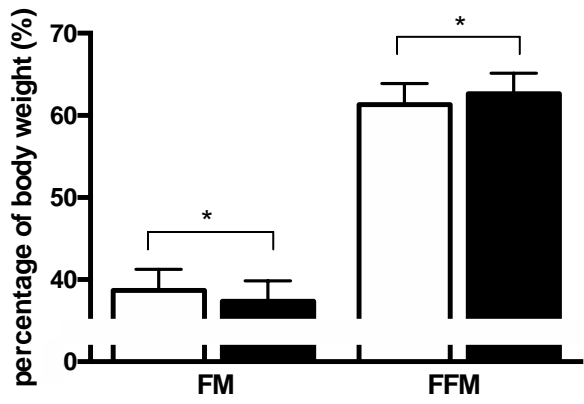

E

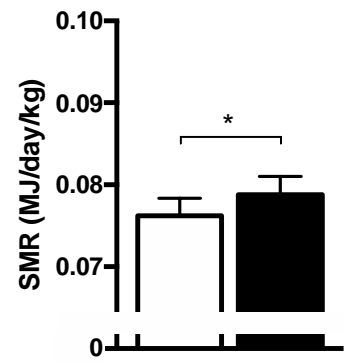

Figure 5: Body composition and sleeping metabolic rate after NR and placebo supplementation. A Bodyweight, fat mass (FM) and fat free mass (FFM) expressed in kilograms (kg). B FM and FFM expressed as percentage (\%) of body weight. C Sleeping metabolic rate expressed (SMR) as MJ per day. D SMR corrected for body weight. E SMR corrected for FFM. Black bars is NR, open bars is placebo. Data are expressed as mean $\pm S E$. $N=13$. $P$ values are derived from the analysis of the mean within-person changes and the SEM of the within-group changes. ${ }^{*} p<0.05$. 
Table 3: Cardiometabolic health parameters

\begin{tabular}{|c|c|c|c|c|}
\hline Parameter & Placebo & NR & Change & p-value \\
\hline \multicolumn{5}{|l|}{ MRS Cardiac left ventricle ${ }^{2}$} \\
\hline Cardiac PCr/ATP ratio & $1.29 \pm 0.11$ & $1.22 \pm 0.09$ & $-0.08 \pm 0.14$ & 0.90 \\
\hline \multicolumn{5}{|l|}{ MRI Cardiac left ventricle ${ }^{1}$} \\
\hline $\mathrm{EF}(\%)$ & $71.2 \pm 2.2$ & $68.2 \pm 2.1$ & $-3.0 \pm 2.4$ & 0.24 \\
\hline ESV (ml) & $35.0 \pm 4.2$ & $38.9 \pm 3.9$ & $4.0 \pm 3.6$ & 0.23 \\
\hline $\operatorname{EDV}(\mathrm{ml})$ & $119.4 \pm 8.2$ & $121.2 \pm 8.3$ & $1.8 \pm 5.1$ & 0.72 \\
\hline $\mathrm{SV}(\mathrm{ml})$ & $84.4 \pm 5.6$ & $82.3 \pm 5.6$ & $-2.1 \pm 3.8$ & 0.69 \\
\hline \multicolumn{5}{|l|}{ Ambulatory BP 36-hour ${ }^{1}$} \\
\hline $\mathrm{SBP}(\mathrm{mmHg})$ & $124.6 \pm 2.4$ & $126.6 \pm 3.2$ & $2.0 \pm 2.3$ & 0.56 \\
\hline $\mathrm{DBP}(\mathrm{mmHg})$ & $77.1 \pm 1.8$ & $77.9 \pm 2.0$ & $0.8 \pm 0.9$ & 0.39 \\
\hline MAP (mmHg) & $98.9 \pm 1.7$ & $100.2 \pm 2.1$ & $1.3 \pm 1.5$ & 0.40 \\
\hline $\mathrm{PP}(\mathrm{mmHg})$ & $47.5 \pm 2.6$ & $48.6 \pm 3.1$ & $1.2 \pm 2.2$ & 0.60 \\
\hline $\mathrm{HR}(\mathrm{bpm})$ & $76.4 \pm 3.3$ & $75.5 \pm 3.6$ & $-0.9 \pm 1.7$ & 0.60 \\
\hline Night time dipping SBP (\%) & $10.6 \pm 2.4$ & $12.4 \pm 0.8$ & $1.8 \pm 2.8$ & 0.53 \\
\hline Night time dipping DBP (\%) & $11.9 \pm 2.4$ & $14.8 \pm 1.9$ & $3.0 \pm 2.5$ & 0.26 \\
\hline \multicolumn{5}{|l|}{ Ambulatory BP daytime } \\
\hline $\mathrm{SBP}(\mathrm{mmHg})$ & $126.9 \pm 2.3$ & $129.0 \pm 2.9$ & $2.1 \pm 2.3$ & 0.54 \\
\hline $\mathrm{DBP}(\mathrm{mmHg})$ & $79.5 \pm 1.7$ & $80.5 \pm 1.9$ & $1.0 \pm 1.0$ & 0.32 \\
\hline MAP (mmHg) & $101.4 \pm 1.5$ & $102.7 \pm 1.9$ & $1.3 \pm 1.4$ & 0.39 \\
\hline $\mathrm{PP}(\mathrm{mmHg})$ & $47.6 \pm 2.7$ & $48.5 \pm 3.0$ & $0.9 \pm 2.1$ & 0.68 \\
\hline $\mathrm{HR}(\mathrm{bpm})$ & $78.3 \pm 3.2$ & $77.5 \pm 3.5$ & $-0.8 \pm 1.7$ & 0.67 \\
\hline \multicolumn{5}{|l|}{ Ambulatory BP night-time ${ }^{1}$} \\
\hline $\mathrm{SBP}(\mathrm{mmHg})$ & $113.5 \pm 3.4$ & $113.0 \pm 3.1$ & $-0.5 \pm 3.7$ & 0.90 \\
\hline $\mathrm{DBP}(\mathrm{mmHg})$ & $69.4 \pm 2.6$ & $68.1 \pm 2.3$ & $-1.3 \pm 1.5$ & 0.41 \\
\hline $\mathrm{MAP}(\mathrm{mmHg})$ & $89.6 \pm 2.7$ & $88.8 \pm 2.4$ & $-0.8 \pm 2.5$ & 0.75 \\
\hline $\mathrm{PP}(\mathrm{mmHg})$ & $43.8 \pm 2.5$ & $45.2 \pm 2.6$ & $1.4 \pm 2.3$ & 0.57 \\
\hline $\mathrm{HR}$ (bpm) & $65.6 \pm 2.5$ & $66.6 \pm 2.9$ & $1.0 \pm 1.9$ & 0.60 \\
\hline
\end{tabular}

PCr, phosphocreatin; ATP, adenosine triphosphate; EF, ejection fraction; ESV, end systolic volume; EDV, end diastolic volume; SV, stroke volume; BP, blood pressure; SBP, systolic blood pressure; DBP, diastolic blood pressure; MAP, mean arterial pressure; PP, pulse pressure; HR, heart rate. ${ }^{1} n=12,{ }^{2} n=11$. Data are expressed as mean $\pm S E$. $P$ values are derived from the analysis of the mean within-person changes and the SE of the withingroup changes.

\section{DISCUSSION}

We hypothesized that NR supplementation in humans would increase $\mathrm{NAD}^{+}$availability and thereby would improve a broad range of metabolic health parameters, mainly via improving mitochondrial function. To investigate this hypothesis, we performed a randomized doubleblinded placebo controlled cross-over study with detailed metabolic phenotyping in which we provided healthy overweight and obese men and women $1000 \mathrm{mg} /$ day NR for six weeks. In line with our hypothesis, NR supplementation did significantly increase markers of NAD ${ }^{+}$ 
metabolism - NAAD and MeNAM - in skeletal muscle. This effect was accompanied by small but statistically significant improvements in body composition, sleeping metabolic rate and skeletal muscle acetylcarnitine concentrations, and a trend towards increased circulatory IL-1 $\alpha$ concentrations. No further effects on skeletal muscle mitochondrial function, hepatic and whole-body insulin sensitivity, substrate oxidation, cardiovascular health markers and ectopic lipid accumulation were observed. However, it should be noted that many outcomes have been tested in our study and no adjustments for multiple comparisons were performed, therefore the possibility of false positive findings cannot be excluded. These results suggest that $\mathrm{NR}$, at the dose of $1000 \mathrm{mg} /$ day for six weeks, did have, albeit relatively small, effects on metabolic parameters in humans, but was not effective in boosting muscle mitochondrial function or insulin sensitivity.

Animal studies showed that NR is able to increase plasma and tissue $\mathrm{NAD}^{+}$concentrations (9-14). Also in humans, NR is able to increase circulatory $N A D^{+}$metabolites after several dosages ranging from $100 \mathrm{mg} /$ day to $2000 \mathrm{mg} /$ day (18-21). Here, we used a dose of 1000 $\mathrm{mg} /$ day for six weeks, and in line with other data presented we did not report side effects $(18,20)$. We investigated if NR supplementation was able to increase the NAD+ metabolome in skeletal muscle tissue. In agreement with findings by Elhassan et al. (20) and Dollerup et al. (24), we show that NR supplementation increased NAD ${ }^{+}$metabolites NAAD and MeNAM in skeletal muscle, but without an increase in total NAD+ content itself. NAAD is a highly sensitive biomarker of $\mathrm{NR}$ supplementation and increased $\mathrm{NAD}^{+}$synthesis rate in tissues (14). MeNAM is part of the NAD ${ }^{+}$degradation pathway and is a marker for increased $N A D^{+}$ flux. These results might suggest that NR increases $N A D^{+}$turnover rate, without affecting steady state $\mathrm{NAD}^{+}$concentrations in skeletal muscle.

We hypothesized that a NR-stimulated increase in $\mathrm{NAD}^{+}$metabolism would lead to an increase in muscle mitochondrial function and a subsequent increase in human insulin sensitivity. However, in contrast to our hypothesis, skeletal muscle mitochondrial function was not elevated upon NR supplementation. This is in agreement with the findings of Elhassan et al. (25) and Dollerup et al. (24), who also reported no effect of NR on skeletal muscle mitochondrial function. Consistent with the lack of effect on mitochondrial function, we and others (21) did not observe improvements on insulin sensitivity upon NR supplementation. Although the limited duration of our and other studies may explain the lack of effect of NR on insulin sensitivity, we have previously shown that nutritional supplements like resveratrol can increase skeletal muscle mitochondrial function after four weeks of supplementation (45-47). Trammel et al. (48) and Dollerup et al. (21) suggested that the underlying pathway of metabolic improvements observed in obese mice was a decrease in hepatic lipid accumulation. In addition, Dollerup et al. (21) described a decrease 
in hepatic lipid content in obese men with elevated baseline hepatic lipid content, although this was not significant. Here, we did not observe an effect of NR on hepatic lipid accumulation, which may be contributed to our study population which had in general a healthy hepatic lipid content (i.e. $<5 \%$ liver fat).

NR supplementation has also been suggested to improve cardiovascular health $(20,49)$. Therefore, we here examined the effect of NR on cardiovascular health via detailed cardiovascular phenotyping. In contrast to Martens et al. (20) but in accordance with Conze et al. (23), we did not observe an effect of NR on blood pressure values. Martens et al. observed a decrease in resting SBP and DBP after NR, whereas we measured 36-hour ambulatory blood pressure, which gives a better estimation of blood pressure values and gives a better indication of the risk of cardiovascular events $(50,51)$. Consistent with the lack of effect of NR on blood pressure we did not find effects of NR on cardiac energy status or cardiac ejection fraction. Of note, the cardiac status of our participants was considered 'healthy', and in rodents also no change in cardiac function after NR supplementation in control mice with a healthy cardiac function could be observed (49).

We reported an improvement in body composition by an increase in percentage fat free mass mirrored by a decrease in percentage fat mass, while body weight remained unchanged. An effect of NR on body composition in humans has not been reported before $(20,21)$. Interestingly, in six out of seven women NR did increase fat free mass and reduce fat mass whereas this was only the case in one out of six men, suggesting that there might be a gender difference in the effect of NR supplementation on body composition. Consistent with the effect of NR on body composition, we show that the sleeping metabolic rate was also affected by NR supplementation and a higher metabolic rate could potentially lead to a reduction in fat mass. The increase in sleeping metabolic rate was due to an increase in fat free mass, suggesting that the primary effect of these findings could be an effect of NR on fat free mass. Interestingly, it has previously been shown that NAD+ metabolism and homeostasis is involved in maintaining muscle mass (13). Future studies should be designed to investigate if NR supplementation can indeed increase muscle mass in humans and investigate the underlying mechanisms.

Next to an effect of NR on muscle mass, NR supplementation enhanced the exerciseinduced increase in acetylcarnitine. Moreover, acetylcarnitine concentrations measured in skeletal muscle biopsies obtained in the morning, showed significantly increased concentrations after NR supplementation. These data suggest that NR is able to increase skeletal muscle acetylcarnitine metabolism, which has been associated with metabolic flexibility and improved metabolic health (36). Remarkably, though, resting acetylcarnitine 
concentrations measured using MRS three hours after lunch and before the exercise session were significantly lower after NR compared to placebo. It has previously been shown that meal consumption lowers acetylcarnitines in skeletal muscle $(52,53)$, but why NR would substantiate such meal-induced lowering in acetylcarnitine metabolism cannot be deducted from this study. Interestingly, a recently published study in obese mice showed an effect of combined supplementation of NR with L-carnitine and reported a reduction in fat mass percentage and hepatic steatosis (54), which matches with the positive outcome parameters of our and other studies $(9,21,48)$. The exact link between NR metabolism and acetylcarnitine metabolism is however still unknown. Furthermore, the large gap between the clear metabolic improvements upon NR in mice and the lack of effects in humans might derive from the fact that mice studies applied a longer supplementation duration ( 8 to 15 weeks) $(9,10,12,48)$ compared to short-term supplementation in human trials (3 to 12 weeks) $(21,24,25,55)$.

In conclusion, we here show that NR supplementation of $1000 \mathrm{mg} /$ day for six weeks in healthy overweight and obese men and women increased the NAD ${ }^{+}$metabolites NAAD and MeNAM in human skeletal muscle and increased skeletal muscle acetylcarnitine metabolism. In addition, NR induced improvements in body composition and increased sleeping metabolic rate. However, no other metabolic health effects were observed. We conclude that NR, at this dose and short duration, may not be beneficial in improving metabolic health in healthy overweight and obese men and women. However, further research is warranted into the effects of long-term NR supplementation on acetylcarnitine concentrations, sex specific improvements in body composition and metabolic health effects in humans. 


\section{ACKNOWLEDGEMENTS}

The authors' contributions were as follows: CR BH JA JH VSH EP PS designed the study. CR KR MM NC VdW conducted the experiments. BH provided medical responsibility. SA BS HE RZP performed analysis in plasma samples and muscle biopsies. CR JM VdW TvdW SA RZP VSH analyzed data. CR SA EL RZP RH JH LL VSH EP PS interpreted the data. CR EP PS wrote paper and all authors reviewed and accepted the last version of the manuscript. PS had primary responsibility for final content. The authors would like to thank the participants for their commitment to this study and ChromaDex Inc. for providing NIAGEN and placebo capsules for the study. PS is the guarantor of this work and, as such, had full access to all the data in the study and takes responsibility for the integrity of the data and the accuracy of the data analysis. The authors declare no conflict of interest relevant to this article. 


\section{Chapter 7}

\section{SUPPLEMENTARY MATERIAL FOR CHAPTER 7}

Supplementary table 1: Internal standard mixture for $\mathrm{NAD}^{+}$targeted metabolomics in skeletal muscle biopsies

\begin{tabular}{|c|c|}
\hline Internal standard & Concentration ( $\mu \mathrm{M})$ \\
\hline Adenosine- ${ }^{15} \mathrm{~N}_{5}$-monophosphate & 100 \\
\hline Adenosine- ${ }^{15} \mathrm{~N}_{5}$-triphosphate & 1000 \\
\hline $\mathrm{D}_{4}$-alanine & 100 \\
\hline $\mathrm{D}_{7}$-arginine & 100 \\
\hline $\mathrm{D}_{3}$-aspartic acid & 100 \\
\hline $\mathrm{D}_{4}$-citric acid & 100 \\
\hline${ }^{13} \mathrm{C}_{1}$-citrulline & 100 \\
\hline${ }^{13} \mathrm{C}_{6}$-fructose-1,6-diphosphate & 100 \\
\hline Guanosine- ${ }^{15} N_{5}$-monophosphate & 100 \\
\hline Guanosine-- ${ }^{15} \mathrm{~N}_{5}$-triphosphate & 1000 \\
\hline${ }^{13} \mathrm{C}_{6}$-glucose & 1000 \\
\hline${ }^{13} C_{6}$-glucose-6-phosphate & 100 \\
\hline $\mathrm{D}_{3}$-glutamic acid & 100 \\
\hline $\mathrm{D}_{5}$-glutamine & 100 \\
\hline${ }^{13} \mathrm{C}_{6}$-isoleucine & 100 \\
\hline $\mathrm{D}_{3}$-leucine & 100 \\
\hline $\mathrm{D}_{4}$-lysine & 100 \\
\hline $\mathrm{D}_{3}$-methionine & 100 \\
\hline $\mathrm{D}_{6}$-ornithine & 100 \\
\hline$D_{5}$-phenylalanine & 100 \\
\hline D7-proline & 100 \\
\hline${ }^{13} \mathrm{C}_{3}$-pyruvate & 100 \\
\hline$D_{3}$-serine & 100 \\
\hline $\mathrm{D}_{5}$-tryptophan & 100 \\
\hline$D_{4}$-tyrosine & 100 \\
\hline D $_{8}$-valine & 100 \\
\hline${ }^{13} C_{5}$-nicotinamide adenine dinucleotide & 100 \\
\hline
\end{tabular}

\section{Supplementary table 2: Participant characteristics}

\begin{tabular}{ll}
\hline Parameter & Mean \pm SD \\
\hline Gender F/M & $7 / 6$ \\
Age (years) & $59 \pm 5$ \\
Body weight $(\mathrm{kg})$ & $87.2 \pm 13.6$ \\
Height $(\mathrm{m})$ & $1.70 \pm 0.11$ \\
$\mathrm{BMI}\left(\mathrm{kg} / \mathrm{m}^{2}\right)$ & $30.2 \pm 2.6$ \\
$\mathrm{VO}_{2}$ peak $(\mathrm{ml} / \mathrm{min} / \mathrm{kg})$ & $27.0 \pm 5.7$ \\
Physical activity level (Baecke score) & $7.51 \pm 1.16$
\end{tabular}

BMI, body mass index; $\mathrm{VO}_{2}$ peak, peak oxygen consumption. 
Supplementary figure 1: Flowchart participant inclusion

\section{Enrollment}

Assessed for eligibility $(n=25)$

Excluded $(n=11)$

- Not meeting inclusion

criteria $(n=8)$

- Declined to participate $(n=3)$

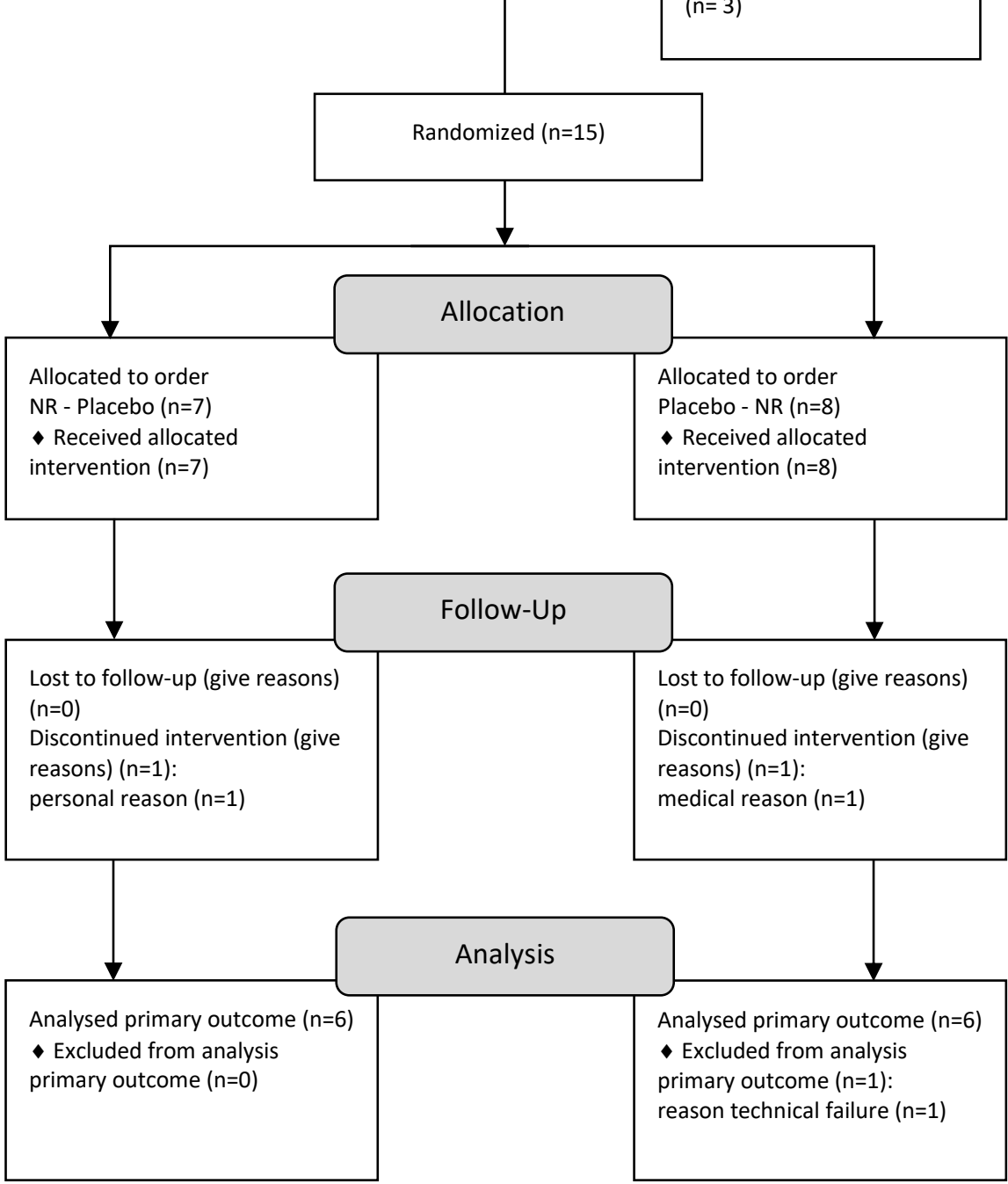




\section{REFERENCES}

1. Bieganowski P, Brenner C. Discoveries of nicotinamide riboside as a nutrient and conserved NRK genes establish a Preiss-Handler independent route to NAD+ in fungi and humans. Cell. 2004;117(4):495-502.

2. Connell NJ, Houtkooper RH, Schrauwen P. NAD(+) metabolism as a target for metabolic health: have we found the silver bullet? Diabetologia. 2019;62(6):88899.

3. Houtkooper RH, Auwerx J. Exploring the therapeutic space around NAD+. J Cell Biol. 2012;199(2):205-9.

4. Imai S, Guarente L. NAD+ and sirtuins in aging and disease. Trends in cell biology. 2014;24(8):464-71.

5. Bonkowski MS, Sinclair DA. Slowing ageing by design: the rise of NAD(+) and sirtuinactivating compounds. Nat Rev Mol Cell Biol. 2016;17(11):679-90.

6. Canto C, Auwerx J. Targeting sirtuin 1 to improve metabolism: all you need is NAD(+)? Pharmacological reviews. 2012;64(1):166-87.

7. Connell NJ, Houtkooper RH, Schrauwen P. NAD(+) metabolism as a target for metabolic health: have we found the silver bullet? Diabetologia. 2019.

8. Fletcher RS, Ratajczak J, Doig CL, Oakey LA, Callingham R, Da Silva Xavier G, Garten A, Elhassan YS, Redpath P, Migaud ME, et al. Nicotinamide riboside kinases display redundancy in mediating nicotinamide mononucleotide and nicotinamide riboside metabolism in skeletal muscle cells. Mol Metab. 2017;6(8):819-32.

9. Canto C, Houtkooper RH, Pirinen E, Youn DY, Oosterveer MH, Cen Y, FernandezMarcos PJ, Yamamoto H, Andreux PA, Cettour-Rose P, et al. The NAD(+) precursor nicotinamide riboside enhances oxidative metabolism and protects against highfat diet-induced obesity. Cell metabolism. 2012;15(6):838-47.

10. Khan NA, Auranen M, Paetau I, Pirinen E, Euro L, Forsstrom S, Pasila L, Velagapudi V, Carroll CJ, Auwerx J, et al. Effective treatment of mitochondrial myopathy by nicotinamide riboside, a vitamin B3. EMBO molecular medicine. 2014;6(6):721-31.

11. Trammell SAJ, Weidemann BJ, Chadda A, Yorek MS, Holmes A, Coppey LJ, Obrosov A, Kardon RH, Yorek MA, Brenner C. Nicotinamide Riboside Opposes Type 2 Diabetes and Neuropathy in Mice.

12. Shi W, Hegeman MA, van Dartel DAM, Tang J, Suarez M, Swarts $H$, van der Hee B, Arola L, Keijer J. Effects of a wide range of dietary nicotinamide riboside (NR) concentrations on metabolic flexibility and white adipose tissue (WAT) of mice fed a mildly obesogenic diet. Mol Nutr Food Res. 2017;61(8).

13. Frederick DW, Loro E, Liu L, Davila A, Jr., Chellappa K, Silverman IM, Quinn WJ, 3rd, Gosai SJ, Tichy ED, Davis JG, et al. Loss of NAD Homeostasis Leads to Progressive and Reversible Degeneration of Skeletal Muscle. Cell metabolism. 2016;24(2):26982.

14. Trammell SA, Schmidt MS, Weidemann BJ, Redpath P, Jaksch F, Dellinger RW, Li Z, Abel ED, Migaud ME, Brenner C. Nicotinamide riboside is uniquely and orally bioavailable in mice and humans. Nature communications. 2016;7:12948. 
15. Yoshino J, Mills KF, Yoon MJ, Imai S. Nicotinamide mononucleotide, a key NAD(+) intermediate, treats the pathophysiology of diet- and age-induced diabetes in mice. Cell metabolism. 2011;14(4):528-36.

16. Massudi H, Grant R, Braidy N, Guest J, Farnsworth B, Guillemin GJ. Age-associated changes in oxidative stress and NAD+ metabolism in human tissue. PloS one. 2012;7(7):e42357.

17. de Guia RM, Agerholm M, Nielsen TS, Consitt LA, Sogaard D, Helge JW, Larsen S, Brandauer J, Houmard JA, Treebak JT. Aerobic and resistance exercise training reverses age-dependent decline in $\mathrm{NAD}(+)$ salvage capacity in human skeletal muscle. Physiol Rep. 2019;7(12):e14139.

18. Dellinger RW, Santos SR, Morris M, Evans M, Alminana D, Guarente L, Marcotulli E. Erratum: Author Correction: Repeat dose NRPT (nicotinamide riboside and pterostilbene) increases $\mathrm{NAD}(+)$ levels in humans safely and sustainably: a randomized, double-blind, placebo-controlled study. NPJ Aging Mech Dis. 2017;4:8.

19. Airhart SE, Shireman LM, Risler LJ, Anderson GD, Nagana Gowda GA, Raftery D, Tian $R$, Shen DD, O'Brien KD. An open-label, non-randomized study of the pharmacokinetics of the nutritional supplement nicotinamide riboside (NR) and its effects on blood NAD+ levels in healthy volunteers. PloS one. 2017;12(12):e0186459.

20. Martens CR, Denman BA, Mazzo MR, Armstrong ML, Reisdorph N, McQueen MB, Chonchol M, Seals DR. Chronic nicotinamide riboside supplementation is welltolerated and elevates NAD+ in healthy middle-aged and older adults. Nature communications.9(1286).

21. Dollerup OL, Christensen B, Svart M, Schmidt MS, Sulek K, Ringgaard S, StodkildeJorgensen $\mathrm{H}$, Moller N, Brenner C, Treebak JT, et al. A randomized placebocontrolled clinical trial of nicotinamide riboside in obese men: safety, insulinsensitivity, and lipid-mobilizing effects. The American journal of clinical nutrition. 2018;108(2):343-53.

22. Conze D, Brenner C, Kruger CL. Safety and Metabolism of Long-term Administration of NIAGEN (Nicotinamide Riboside Chloride) in a Randomized, Double-Blind, Placebo-controlled Clinical Trial of Healthy Overweight Adults. Sci Rep. 2019;9(1):9772.

23. Conze DB, Crespo-Barreto J, Kruger CL. Safety assessment of nicotinamide riboside, a form of vitamin B3. Human \& experimental toxicology. 2016.

24. Dollerup OL, Chubanava S, Agerholm M, Søndergard SD, Altintas A, Møller AB, Høyer KF, Ringgaard S, Stødkilde-Jørgensen H, Lavery GG, et al. Nicotinamide riboside does not alter mitochondrial respiration, content or morphology in skeletal muscle from obese and insulin resistant men. The Journal of physiology. 2019.

25. Elhassan YS, Kluckova K, Fletcher RS, Schmidt MS, Garten A, Doig CL, Cartwright DM, Oakey L, Burley CV, Jenkinson N, et al. Nicotinamide Riboside Augments the Aged Human Skeletal Muscle NAD(+) Metabolome and Induces Transcriptomic and Anti-inflammatory Signatures. Cell reports. 2019;28(7):1717-28 e6. 
26. Baecke JA, Burema J, Frijters JE. A short questionnaire for the measurement of habitual physical activity in epidemiological studies. The American journal of clinical nutrition. 1982;36(5):936-42.

27. Defronzo RA, Tobin JD, Andres R. Glucose clamp technique: a method for quantifying insulin secretion and resistance. Am J Physiol. 1979;237(3):E214-23.

28. Bergstrom J, Hermansen L, Hultman E, Saltin B. Diet, muscle glycogen and physical performance. Acta physiologica Scandinavica. 1967;71(2):140-50.

29. Kato T, Berger SJ, Carter JA, Lowry OH. An enzymatic cycling method for nicotinamide-adenine dinucleotide with malic and alcohol dehydrogenases. Analytical biochemistry. 1973;53(1):86-97.

30. van de Weijer T, Phielix E, Bilet L, Williams EG, Ropelle ER, Bierwagen A, Livingstone $R$, Nowotny $P$, Sparks LM, Paglialunga $S$, et al. Evidence for a direct effect of the $\mathrm{NAD}+$ precursor acipimox on muscle mitochondrial function in humans. Diabetes. 2015;64(4):1193-201.

31. Hoeks J, van Herpen NA, Mensink M, Moonen-Kornips E, van Beurden D, Hesselink MK, Schrauwen P. Prolonged fasting identifies skeletal muscle mitochondrial dysfunction as consequence rather than cause of human insulin resistance. Diabetes. 2010;59(9):2117-25.

32. Wefers J, van Moorsel D, Hansen J, Connell NJ, Havekes B, Hoeks J, van Marken Lichtenbelt WD, Duez H, Phielix E, Kalsbeek A, et al. Circadian misalignment induces fatty acid metabolism gene profiles and compromises insulin sensitivity in human skeletal muscle. Proceedings of the National Academy of Sciences of the United States of America. 2018;115(30):7789-94.

33. Kuipers H, Verstappen FT, Keizer HA, Geurten P, van Kranenburg G. Variability of aerobic performance in the laboratory and its physiologic correlates. Int J Sports Med. 1985;6(4):197-201.

34. Lindeboom L, Nabuurs Cl, Hesselink MK, Wildberger JE, Schrauwen P, SchrauwenHinderling VB. Proton magnetic resonance spectroscopy reveals increased hepatic lipid content after a single high-fat meal with no additional modulation by added protein. The American journal of clinical nutrition. 2015;101(1):65-71.

35. Lindeboom L, Bruls YM, van Ewijk PA, Hesselink MK, Wildberger JE, Schrauwen $P$, Schrauwen-Hinderling VB. Longitudinal relaxation time editing for acetylcarnitine detection with (1) H-MRS. Magn Reson Med. 2017;77(2):505-10.

36. Lindeboom L, Nabuurs Cl, Hoeks J, Brouwers B, Phielix E, Kooi ME, Hesselink MK, Wildberger JE, Stevens RD, Koves T, et al. Long-echo time MR spectroscopy for skeletal muscle acetylcarnitine detection. The Journal of clinical investigation. 2014;124(11):4915-25.

37. van de Weijer T, van Ewijk PA, Zandbergen HR, Slenter JM, Kessels AG, Wildberger JE, Hesselink MK, Schrauwen P, Schrauwen-Hinderling VB, Kooi ME. Geometrical models for cardiac MRI in rodents: comparison of quantification of left ventricular volumes and function by various geometrical models with a full-volume MRI data set in rodents. Am J Physiol Heart Circ Physiol. 2012;302(3):H709-15.

38. Joris PJ, Plat J, Bakker SJ, Mensink RP. Long-term magnesium supplementation improves arterial stiffness in overweight and obese adults: results of a randomized, 
double-blind, placebo-controlled intervention trial. The American journal of clinical nutrition. 2016;103(5):1260-6.

39. Dempster P, Aitkens S. A new air displacement method for the determination of human body composition. Med Sci Sports Exerc. 1995;27(12):1692-7.

40. Plasqui G, Soenen S, Westerterp-Plantenga MS, Westerterp KR. Measurement of longitudinal changes in body composition during weight loss and maintenance in overweight and obese subjects using air-displacement plethysmography in comparison with the deuterium dilution technique. Int J Obes (Lond). 2011;35(8):1124-30.

41. Friedewald WT, Levy RI, Fredrickson DS. Estimation of the concentration of lowdensity lipoprotein cholesterol in plasma, without use of the preparative ultracentrifuge. Clin Chem. 1972;18(6):499-502.

42. Peronnet $F$, Massicotte $D$. Table of nonprotein respiratory quotient: an update. Can J Sport Sci. 1991;16(1):23-9.

43. Weir JB. New methods for calculating metabolic rate with special reference to protein metabolism. The Journal of physiology. 1949;109(1-2):1-9.

44. Steele R. Influences of glucose loading and of injected insulin on hepatic glucose output. Annals of the New York Academy of Sciences. 1959;82:420-30.

45. de Ligt M, Bruls YMH, Hansen J, Habets MF, Havekes B, Nascimento EBM, MoonenKornips E, Schaart G, Schrauwen-Hinderling VB, van Marken Lichtenbelt W, et al. Resveratrol improves ex vivo mitochondrial function but does not affect insulin sensitivity or brown adipose tissue in first degree relatives of patients with type 2 diabetes. Mol Metab. 2018;12:39-47.

46. Timmers S, Konings E, Bilet L, Houtkooper RH, van de Weijer T, Goossens GH, Hoeks $\mathrm{J}$, van der Krieken S, Ryu D, Kersten S, et al. Calorie restriction-like effects of 30 days of resveratrol (resVida) supplementation on energy metabolism and metabolic profile in obese humans. Cell metabolism. 2011;14(5).

47. Timmers $S$, de Ligt M, Phielix E, van de Weijer $T$, Hansen J, Moonen-Kornips E, Schaart G, Kunz I, Hesselink MK, Schrauwen-Hinderling VB, et al. Resveratrol as Add-on Therapy in Subjects With Well-Controlled Type 2 Diabetes: A Randomized Controlled Trial. Diabetes care. 2016;39:2211-7.

48. Trammell SA, Weidemann BJ, Chadda A, Yorek MS, Holmes A, Coppey LJ, Obrosov A, Kardon RH, Yorek MA, Brenner C. Nicotinamide Riboside Opposes Type 2 Diabetes and Neuropathy in Mice. Sci Rep. 2016;6:26933.

49. Diguet N, Trammell SAJ, Tannous C, Deloux R, Piquereau J, Mougenot N, Gouge A, Gressette M, Manoury B, Blanc J, et al. Nicotinamide Riboside Preserves Cardiac Function in a Mouse Model of Dilated Cardiomyopathy. Circulation. 2018;137(21):2256-73.

50. Verdecchia P, Angeli F, Cavallini C. Ambulatory blood pressure for cardiovascular risk stratification. Circulation. 2007;115(16):2091-3.

51. Hansen TW, Jeppesen J, Rasmussen S, Ibsen H, Torp-Pedersen C. Ambulatory blood pressure monitoring and risk of cardiovascular disease: a population based study. Am J Hypertens. 2006;19(3):243-50. 
52. Klepochova R, Valkovic L, Gajdosik M, Hochwartner T, Tschan H, Krebs M, Trattnig $S$, Krssak M. Detection and Alterations of Acetylcarnitine in Human Skeletal Muscles by $1 \mathrm{H}$ MRS at 7 T. Invest Radiol. 2017;52(7):412-8.

53. Watt MJ, Heigenhauser GJ, Stellingwerff T, Hargreaves M, Spriet LL. Carbohydrate ingestion reduces skeletal muscle acetylcarnitine availability but has no effect on substrate phosphorylation at the onset of exercise in man. The Journal of physiology. 2002;544(3):949-56.

54. Salic K, Gart E, Seidel F, Verschuren L, Caspers M, van Duyvenvoorde W, Wong KE, Keijer J, Bobeldijk-Pastorova I, Wielinga PY, et al. Combined treatment with Icarnitine and nicotinamide riboside improves hepatic metabolism and attenuates obesity and liver steatosis. International journal of molecular sciences. 2019;20:4359.

55. Martens CR, Denman BA, Mazzo MR, Armstrong ML, Reisdorph N, McQueen MB, Chonchol $M$, Seals DR. Chronic nicotinamide riboside supplementation is welltolerated and elevates $\mathrm{NAD}(+)$ in healthy middle-aged and older adults. Nature communications. 2018;9(1):1286. 
NR supplementation 
CHAPTER 8

General discussion and conclusion 
NAFL is the number one chronic liver condition worldwide (1). It is estimated that $25 \%$ of the adult population worldwide has NAFL (1). In obesity, NAFL rates are even higher, with reported prevalence ranging from $55 \%$ to $75 \%(2,3)$. Fatty liver can progress into more severe conditions such as NASH and cirrhosis, which will ultimately increase the risk for hepatocellular carcinoma (4). The negative consequences of NAFL are however not only related to progressive liver disease, but also include cardiometabolic effects. In this respect, NAFL has been associated with cardiovascular disease and type 2 diabetes $(5,6)$. People with NAFL have been shown to have a reduced whole-body and hepatic insulin resistance, which is a major risk factor in the development of T2D (7-10). Little is known about the disturbances in substrate metabolism of the liver in NAFL even though such disturbances could play an important role in NAFL etiology and its contribution to cardiometabolic health. To study hepatic substrate metabolism and substrate storage, advanced (MR) methodologies can be instrumental. Therefore, the research in this $\mathrm{PhD}$ thesis focusses on the use of advanced magnetic resonance (MR) methodology to investigate hepatic substrate metabolism and to better understand the metabolic changes that take place during the development of NAFL. Specifically, the importance of hepatic lipid composition (i.e. lipid saturation) and hepatic glycogen are investigated.

\section{How to measure different substrate stores in NAFL?}

A technique that is of great value in determining hepatic substrate stores non-invasively is magnetic resonance spectroscopy (MRS). Using ${ }^{1} \mathrm{H}-\mathrm{MRS}$, information on intrahepatic lipid (IHL) storage can be acquired, whereas ${ }^{13} \mathrm{C}-\mathrm{MRS}$ can be used to determine hepatic glycogen storage.

IHL per se has been negatively associated with metabolic health. However, there are also examples of dissociation between IHL and insulin sensitivity. For instance, increased ChREBP has been shown to promote IHL accumulation without worsening insulin sensitivity (11) and the presence of the rs738409 SNP in the PNPLA3 gene positively associates with hepatic fat storage, without being associated with insulin resistance (12). There is evidence that lipid composition may even be more important than content per se: the type of stored fat may also influence metabolic health. A high degree of palmitic (C16:0) acid in serum has been shown to be associated with impaired insulin sensitivity (13). In addition, differences in lipid composition have been shown in liver biopsies of people with and without NAFL $(14,15)$. Furthermore, high fractions of hepatic saturated fatty acids (SFA) might explain the associations seen between de novo lipogenesis (DNL) and poor metabolic health (16-18), as SFA is the main end product of DNL. 
Therefore, determining liver fat composition (determination of SFA, monounsaturated fatty acids (MUFA) and polyunsaturated fatty acids (PUFA)) in humans is of great interest. As taking biopsies to determine fat composition is not preferred because of its invasiveness, MRS could be applied as an excellent non-invasive alternative. Determining the hepatic fatty acid fractions is however challenging, because: 1 ) the resonances of unsaturated fatty acids are small, 2) resonances overlap and 3) imperfect suppression of the large water resonance leads to contamination of other resonances. In chapter 3, we set-up, validated and applied a novel MRS technique to non-invasively determine the fraction of hepatic SFA, MUFA and PUFA separately in humans, while addressing all the beforementioned difficulties. We validated our approach in oils with high resolution ${ }^{13} \mathrm{C}-\mathrm{NMR}$, in human subcutaneous adipose tissue by comparing MRS determined fat composition to MS-based values in biopsies, and by showing good reproducibility in liver in humans. This novel methodology enabled us to determine liver fat composition in healthy and metabolically compromised volunteers. Importantly, by using this new methodology, we could also determine the relation of the hepatic fatty acid composition to hepatic insulin sensitivity and de novo lipogenesis (DNL).

When quantifying $\mathrm{IHL}$ content per se one should keep in mind that different factors could influence the quantification. Determining IHL content requires accurate $T_{2}$ correction of MRS signals. Commonly, fixed water and lipid $T_{2}$ values are used (19-21). However, $T_{2}$ relaxation times might differ with increased levels of hepatic steatosis and therefore could influence IHL quantification. In chapter 6 we therefore examined how variations in $T_{2}$ relaxation times affect the calculations of absolute $I H L \%(w / w)$. We showed that $T_{2}$ relaxation times are different with different levels of steatosis. Correction with a fixed average $T_{2}$ is introducing only very minor error as long as the investigated population is homogenous in terms of steatosis. However, in individuals with different levels of steatosis, using fixed average $T_{2}$ correction factors lead to an overestimation of liver fat content in people with high IHL content. In general, it would be the best approach to determine the $T_{2}$ relaxation times individually in each volunteer in order to prevent any bias in the calculations of IHL. This strategy however requires additional acquisitions of MR spectra at multiple echo times, which is time consuming. We showed that by using STEAM sequence with short echo time the bias in IHL content was minimized.

Combining ${ }^{1} \mathrm{H}$-MRS with ${ }^{13} \mathrm{C}$-MRS provides the opportunity to acquire additional information on liver substrate stores and metabolism, giving a broad picture of liver substrate metabolism, which can aid in elucidating NAFL etiology. ${ }^{13} \mathrm{C}-\mathrm{MRS}$ can be used to determine hepatic glycogen content (22-26), another substrate that may be a key factor in determining substrate metabolism, specifically partitioning of fat towards oxidation or 
storage. Hepatic glycogen changes rhythmically during the day, with increased levels postprandially and decreasing glycogen levels during fasting periods (22-24). Little is known about the dynamics of glycogen and its effects on fat and carbohydrate metabolism in NAFL. We used ${ }^{13} \mathrm{C}$-MRS in chapter 4 to determine the effect of one-night prolonged fasting on hepatic glycogen levels in individuals with NAFL.

\section{How are liver substrate stores changed in NAFL?}

IHL content is (by definition) increased in NAFL, however, next to this, also carbohydrate metabolism and glycogen storage may be affected and the application of both ${ }^{1} \mathrm{H}$-MRS and ${ }^{13} \mathrm{C}$-MRS can give a broad picture of hepatic metabolism. We applied our novel ${ }^{1} \mathrm{H}-\mathrm{MRS}$ approach and ${ }^{13} \mathrm{C}$-MRS to determine hepatic lipid composition and hepatic glycogen and elucidated their role in NAFL.

As expected, we found a strong positive correlation between DNL and hepatic SFA fraction (chapter 3). In contrast, MUFA fraction negatively correlated with DNL. These results suggest that the formation of SFA in the liver is not necessarily accompanied by subsequent desaturation to MUFA, as is generally believed. Indeed, animal studies do show upregulation of desaturation markers when lipogenic enzymes are upregulated (11); this may suggest that the formation of SFA from DNL may be handled differently in animals and humans. The positive association between DNL and hepatic SFA fraction was further confirmed by our finding that patients with glycogen storage disease type 1a (GSD1a), a genetic model of increased DNL (27), showed an increased hepatic SFA fraction compared to healthy overweight or obese volunteers. Moreover, the hepatic SFA fraction was also increased in people with NAFL and T2D patients, two populations that have been identified with high DNL rates before $(16,28,29)$.

Next to changes in liver fat composition in NAFL, we also investigated hepatic glycogen in chapter 4, as another substrate that may serve as a key factor in the development of NAFL. We tested our hypothesis that an overnight reduction in hepatic glycogen would become more prominent when prolonging the overnight fast by 6.5 hours, in volunteers with NAFL. To this end, two protocols were used: an early dinner with a subsequent long fast and a late dinner with shorter overnight fast. Surprisingly, we found no significant reduction in hepatic glycogen either in the short or prolonged overnight fast. This indicates that the use of hepatic glycogen is reduced in people with NAFL. In chapter 5 we modulated IHL content by decreasing dietary glycemic index (GI) and dietary SFA content, while macronutrient composition was kept similar, and investigated whether changes in hepatic glycogen were underlying these differences in IHL content. Decreasing dietary GI and SFA content did 
however not affect hepatic glycogen levels in the morning after an overnight fast. Possibly, differences in glycogen levels that might have been present over the day were masked by the predominating effect of the overnight fast on reducing glycogen levels. From our studies it appears that in NAFL hepatic SFA fraction is increased and hepatic glycogen cycling is reduced. Reduced hepatic glycogen cycling could be a mechanism underlying the development of NAFL and increased saturation of hepatic fat, mediated through increased rates of DNL. A link between hepatic glycogen content and DNL has been suggested before. Overfeeding studies with carbohydrates show that carbohydrates are first used to replenish glycogen stores and when these glycogen stores have reached their maximal capacity DNL and carbohydrate oxidation are increased (30). Other indications can be found in GSD1a patients, which are characterised by increased rates of DNL as glycogen breakdown is hampered by a defect in glucose-6-phosphatase (27) and we show in chapter 3 that these patients also have an increased hepatic SFA fraction. This connection between hepatic glycogen stores and DNL is also apparent in mice unable to synthesize glycogen, shuttling carbohydrates to the DNL pathway instead and end up with hepatic steatosis (31). Apart from a link to DNL, hepatic glycogen may also influence fat oxidation. Whole-body fat oxidation on a high-fat diet has been shown to be increased when glycogen levels are lowered by exhaustive exercise, as compared to a high-fat diet without preceding glycogenlowering exercise (32). In chapter 4 we show that during overnight fasting whole-body fat oxidation did not increase in individuals with NAFL, in line with the absence of fluctuations in hepatic glycogen. Such metabolic inflexibility has earlier also been shown in volunteers with pre-diabetes (33). Thus, a decreased cycling of hepatic glycogen as indicated by the results of chapter 4 could promote DNL and reduce the switch to fat oxidation, both contributing to increased storage of hepatic fat, that will be relatively high in SFA. Furthermore, in chapter $\mathbf{5}$ we showed that even in a healthy overweight population, not defined as prediabetic or having NAFL, the shift from carbohydrate to fat oxidation that is seen in healthy individuals does not take place during the night. This suggests that the inertia in metabolic flexibility takes place early in the development of metabolic disease, and therefore can be an essential target for interventions.

\section{What are the metabolic consequences of altered substrate metabolism in NAFL?}

As we have shown that hepatic SFA is increased in NAFL, possibly influenced by reduced fluctuations in hepatic glycogen, the question arises what the metabolic consequences of increased hepatic SFA are. Therefore, we investigated in chapter $\mathbf{3}$ whether liver fat composition correlates to hepatic insulin sensitivity. We showed that the hepatic SFA fraction was negatively related to hepatic insulin sensitivity and that this correlation was 
stronger than for total IHL. This suggests that it is the type of fat that is of great importance in determining hepatic insulin sensitivity, rather than the total IHL content. As we have also shown in chapter 3 that the hepatic SFA fraction is a reflection of DNL and it has been shown before that also DNL is associated with hepatic insulin resistance (34), the question arises whether hepatic insulin resistance is due to the process of DNL or whether the accumulation of SFA per se causes hepatic insulin resistance. Diets high in SFA have been associated with decreased metabolic health before (35-37). Reducing the amount of dietary SFA may therefore have a therapeutic effect on metabolic health, as we showed in chapter $\mathbf{5}$ by improvements in IHL content and glycemic response. The proposed question is of clinical relevance, as it will give novel insights towards the best strategy for prevention and treatment of hepatic insulin resistance. Next to interventions targeting DNL, such as low carbohydrate, low fructose and low GI diets or drugs such as ACC inhibitors, diets targeting liver fat saturation directly could be effective. Indeed, increased desaturation following lipogenesis is believed to be a rescue mechanism to reduce the negative effects exerted by SFA (11). Thus, use of drugs targeting liver fat saturation might be an effective strategy to improve metabolic health in people with NAFL and T2D. It is well-known that hepatic desaturases are under the control of PPAR and therefore, PPARs may be a potential drug target. PPAR $\alpha / \delta$ stimulation has previously been shown to improve fasting plasma glucose, whole-body insulin sensitivity and especially hepatic insulin sensitivity $(38,39)$. Strikingly, these effects seem to be independent of reductions in liver fat per se and thus PPARs may improve metabolic health and specifically insulin resistance via decreased liver fat saturation.

\section{Future perspectives}

Using our novel MRS methodology, we showed that the hepatic SFA fraction is a reflection of DNL and that specifically this fraction may hamper hepatic insulin sensitivity (chapter 3 ). It is however important to keep in mind that these results are only associative and future research will have to show whether decreasing the amount of SFA in the liver has beneficial effects on metabolic health. Studies targeting hepatic fat saturation by diets or drugs could provide an answer to the question whether the relationship between hepatic SFA and hepatic insulin sensitivity is causal. If so, the use of PPAR agonists might prove to be an effective strategy to decrease hepatic fat saturation and thereby improve hepatic insulin sensitivity. These strategies, targeting hepatic SFA, will need to be investigated in the future. They are however only effective if the negative metabolic effects are mediated by hepatic SFA per se. In case increased hepatic SFA would merely be a marker of increased DNL and the process of DNL seems to be mediating the observed negative effects on hepatic insulin 
sensitivity, interventions should specifically target DNL instead. To answer this pivotal question, the metabolic effects of interventions targeting DNL and hepatic SFA per se need to be compared. This can be done by dietary means, using a high fructose diet targeting DNL versus a high SFA diet increasing the hepatic SFA fraction per se.

Another interesting finding in this thesis that requires more investigation is the lack of change in hepatic glycogen upon prolonged overnight fasting in people with NAFL (chapter 4). This needs to be investigated in more detail to determine whether stimulating hepatic glycogen cycling is beneficial. It could be that specifically in NAFL, fluctuations in glycogen are reduced and that this is underlying the excessive storage of hepatic fat. It would therefore first of all be interesting to study how hepatic glycogen levels respond in healthy individuals without NAFL when following a similar prolonged fasting intervention as used in chapter 4. Furthermore, measuring gluconeogenesis and gluconeogenic substrate availability in healthy volunteers and volunteers with NAFL may help us to understand the complex metabolic interplay in NAFL. Increased hepatic gluconeogenesis, that could be driven by blunted postprandial inhibition of lipolysis, might be used as a source for hepatic glucose and thereby prevent the need for the use of hepatic glycogen overnight. To investigate whether hepatic gluconeogenesis is already elevated in the early fed-to-fasting transition period and whether this results in a blunted decline in hepatic glycogen overnight, hepatic gluconeogenesis and hepatic glycogen stores could be measured in healthy volunteers and volunteers with NAFL in the late evening and in the morning. 


\section{REFERENCES}

1. Younossi Z, Tacke F, Arrese M, Chander Sharma B, Mostafa I, Bugianesi E, et al. Global Perspectives on Nonalcoholic Fatty Liver Disease and Nonalcoholic Steatohepatitis. Hepatology (Baltimore, Md). 2019;69(6):2672-82.

2. Gaggini M, Morelli M, Buzzigoli E, DeFronzo RA, Bugianesi E, Gastaldelli A. Nonalcoholic fatty liver disease (NAFLD) and its connection with insulin resistance, dyslipidemia, atherosclerosis and coronary heart disease. Nutrients. 2013;5(5):1544-60.

3. Bellentani S, Saccoccio G, Masutti F, Croce LS, Brandi G, Sasso F, et al. Prevalence of and risk factors for hepatic steatosis in Northern Italy. Annals of internal medicine. 2000;132(2):112-7.

4. Calzadilla Bertot L, Adams LA. The Natural Course of Non-Alcoholic Fatty Liver Disease. International journal of molecular sciences. 2016;17(5).

5. Mantovani A, Byrne CD, Bonora E, Targher G. Nonalcoholic Fatty Liver Disease and Risk of Incident Type 2 Diabetes: A Meta-analysis. Diabetes care. 2018;41(2):37282.

6. Targher G, Byrne CD, Lonardo A, Zoppini G, Barbui C. Non-alcoholic fatty liver disease and risk of incident cardiovascular disease: A meta-analysis. Journal of hepatology. 2016;65(3):589-600.

7. Lim S, Oh TJ, Koh KK. Mechanistic link between nonalcoholic fatty liver disease and cardiometabolic disorders. International journal of cardiology. 2015;201:408-14.

8. Targher G, Byrne CD. Clinical Review: Nonalcoholic fatty liver disease: a novel cardiometabolic risk factor for type 2 diabetes and its complications. The Journal of clinical endocrinology and metabolism. 2013;98(2):483-95.

9. Targher G, Day CP, Bonora E. Risk of cardiovascular disease in patients with nonalcoholic fatty liver disease. The New England journal of medicine. 2010;363(14):1341-50.

10. Brouwers B, Schrauwen-Hinderling VB, Jelenik T, Gemmink A, Havekes B, Bruls Y, et al. Metabolic disturbances of non-alcoholic fatty liver resemble the alterations typical for type 2 diabetes. Clinical science (London, England : 1979). 2017;131(15):1905-17.

11. Benhamed F, Denechaud PD, Lemoine M, Robichon C, Moldes M, Bertrand-Michel $\mathrm{J}$, et al. The lipogenic transcription factor ChREBP dissociates hepatic steatosis from insulin resistance in mice and humans. The Journal of clinical investigation. 2012;122(6):2176-94.

12. Romeo S, Kozlitina J, Xing C, Pertsemlidis A, Cox D, Pennacchio LA, et al. Genetic variation in PNPLA3 confers susceptibility to nonalcoholic fatty liver disease. Nature genetics. 2008;40(12):1461-5.

13. Vessby B, Gustafsson IB, Tengblad S, Boberg M, Andersson A. Desaturation and elongation of Fatty acids and insulin action. Annals of the New York Academy of Sciences. 2002;967:183-95.

14. Puri P, Baillie RA, Wiest MM, Mirshahi F, Choudhury J, Cheung O, et al. A lipidomic analysis of nonalcoholic fatty liver disease. Hepatology (Baltimore, Md). 2007;46(4):1081-90. 
15. Araya J, Rodrigo R, Videla LA, Thielemann L, Orellana M, Pettinelli $P$, et al. Increase in long-chain polyunsaturated fatty acid $n-6 / n-3$ ratio in relation to hepatic steatosis in patients with non-alcoholic fatty liver disease. Clinical science (London, England : 1979). 2004;106(6):635-43.

16. Lambert JE, Ramos-Roman MA, Browning JD, Parks EJ. Increased de novo lipogenesis is a distinct characteristic of individuals with nonalcoholic fatty liver disease. Gastroenterology. 2014;146(3):726-35.

17. Gluchowski NL, Gabriel KR, Chitraju C, Bronson RT, Mejhert N, Boland S, et al. Hepatocyte Deletion of Triglyceride-Synthesis Enzyme Acyl CoA: Diacylglycerol Acyltransferase 2 Reduces Steatosis Without Increasing Inflammation or Fibrosis in Mice. Hepatology (Baltimore, Md). 2019;70(6):1972-85.

18. Matikainen N, Adiels M, Söderlund S, Stennabb S, Ahola T, Hakkarainen A, et al. Hepatic lipogenesis and a marker of hepatic lipid oxidation, predict postprandial responses of triglyceride-rich lipoproteins. Obesity (Silver Spring, Md). 2014;22(8):1854-9.

19. Szczepaniak LS, Nurenberg P, Leonard D, Browning JD, Reingold JS, Grundy S, et al. Magnetic resonance spectroscopy to measure hepatic triglyceride content: prevalence of hepatic steatosis in the general population. American journal of physiology Endocrinology and metabolism. 2005;288(2):E462-8.

20. Guiu B, Petit JM, Loffroy R, Ben Salem D, Aho S, Masson D, et al. Quantification of liver fat content: comparison of triple-echo chemical shift gradient-echo imaging and in vivo proton MR spectroscopy. Radiology. 2009;250(1):95-102.

21. Lindeboom L, Nabuurs Cl, Hesselink MK, Wildberger JE, Schrauwen P, SchrauwenHinderling VB. Proton magnetic resonance spectroscopy reveals increased hepatic lipid content after a single high-fat meal with no additional modulation by added protein. The American journal of clinical nutrition. 2015;101(1):65-71.

22. Magnusson I, Rothman DL, Katz LD, Shulman RG, Shulman GI. Increased rate of gluconeogenesis in type II diabetes mellitus. A $13 \mathrm{C}$ nuclear magnetic resonance study. The Journal of clinical investigation. 1992;90(4):1323-7.

23. Krssak M, Brehm A, Bernroider E, Anderwald C, Nowotny P, Dalla Man C, et al. Alterations in postprandial hepatic glycogen metabolism in type 2 diabetes. Diabetes. 2004;53(12):3048-56.

24. Macauley M, Smith FE, Thelwall PE, Hollingsworth KG, Taylor R. Diurnal variation in skeletal muscle and liver glycogen in humans with normal health and Type 2 diabetes. Clinical science (London, England : 1979). 2015;128(10):707-13.

25. Taylor R, Magnusson I, Rothman DL, Cline GW, Caumo A, Cobelli C, et al. Direct assessment of liver glycogen storage by $13 \mathrm{C}$ nuclear magnetic resonance spectroscopy and regulation of glucose homeostasis after a mixed meal in normal subjects. The Journal of clinical investigation. 1996;97(1):126-32.

26. Bawden S, Stephenson M, Falcone Y, Lingaya M, Ciampi E, Hunter K, et al. Increased liver fat and glycogen stores after consumption of high versus low glycaemic index food: A randomized crossover study. Diabetes, obesity \& metabolism. 2017;19(1):70-7. 
27. Bandsma RH, Prinsen BH, van Der Velden Mde S, Rake JP, Boer T, Smit GP, et al. Increased de novo lipogenesis and delayed conversion of large VLDL into intermediate density lipoprotein particles contribute to hyperlipidemia in glycogen storage disease type 1a. Pediatr Res. 2008;63(6):702-7.

28. Donnelly KL, Smith Cl, Schwarzenberg SJ, Jessurun J, Boldt MD, Parks EJ. Sources of fatty acids stored in liver and secreted via lipoproteins in patients with nonalcoholic fatty liver disease. The Journal of clinical investigation. 2005;115(5):1343-51.

29. Schwarz JM, Linfoot P, Dare D, Aghajanian K. Hepatic de novo lipogenesis in normoinsulinemic and hyperinsulinemic subjects consuming high-fat, lowcarbohydrate and low-fat, high-carbohydrate isoenergetic diets. The American journal of clinical nutrition. 2003;77(1):43-50.

30. Acheson KJ, Schutz Y, Bessard T, Anantharaman K, Flatt JP, Jequier E. Glycogen storage capacity and de novo lipogenesis during massive carbohydrate overfeeding in man. The American journal of clinical nutrition. 1988;48(2):240-7.

31. Irimia JM, Meyer CM, Segvich DM, Surendran S, DePaoli-Roach AA, Morral N, et al. Lack of liver glycogen causes hepatic insulin resistance and steatosis in mice. The Journal of biological chemistry. 2017;292(25):10455-64.

32. Schrauwen $P$, van Marken Lichtenbelt WD, Saris WH, Westerterp KR. Role of glycogen-lowering exercise in the change of fat oxidation in response to a high-fat diet. The American journal of physiology. 1997;273(3 Pt 1):E623-9.

33. Wefers J, Connell NJ, Fealy CE, Andriessen C, de Wit V, van Moorsel D, et al. Daynight rhythm of skeletal muscle metabolism is disturbed in older, metabolically compromised individuals. Molecular metabolism. 2020;41:101050.

34. Low WS, Cornfield T, Charlton CA, Tomlinson JW, Hodson L. Sex Differences in Hepatic De Novo Lipogenesis with Acute Fructose Feeding. Nutrients. 2018;10(9).

35. Parry SA, Rosqvist F, Mozes FE, Cornfield T, Hutchinson M, Piche ME, et al. Intrahepatic Fat and Postprandial Glycemia Increase After Consumption of a Diet Enriched in Saturated Fat Compared With Free Sugars. Diabetes care. 2020.

36. Rosquist F, McNeil CA, Pramfalk C, Parry SA, Low WS, Cornfield T, et al. Fasting hepatic de novo lipogenesis is not reliably assessed using circulating fatty acid markers. The American journal of clinical nutrition. 2019;109(2):260-8.

37. Rosqvist F, Iggman D, Kullberg J, Cedernaes J, Johansson HE, Larsson A, et al. Overfeeding polyunsaturated and saturated fat causes distinct effects on liver and visceral fat accumulation in humans. Diabetes. 2014;63(7):2356-68.

38. Cariou B, Zaï $Y$, Staels B, Bruckert E. Effects of the new dual PPAR $\alpha / \delta$ agonist GFT505 on lipid and glucose homeostasis in abdominally obese patients with combined dyslipidemia or impaired glucose metabolism. Diabetes care. 2011;34(9):2008-14.

39. Cariou B, Hanf R, Lambert-Porcheron S, Zaïr $Y$, Sauvinet $V$, Noël B, et al. Dual peroxisome proliferator-activated receptor $\alpha / \delta$ agonist GFT505 improves hepatic and peripheral insulin sensitivity in abdominally obese subjects. Diabetes care. 2013;36(10):2923-30. 
General discussion 


\title{
Addendum
}

\author{
IMPACT \\ SUMMARY \\ SAMENVATTING \\ ABOUT THE AUTHOR \\ LIST OF PUBLICATIONS \\ DANKWOORD
}




\section{IMPACT}

\section{What is the main aim of the research described in this thesis and what are the most important results and conclusions?}

The aim of this thesis is to investigate how fat and glucose metabolism are changed in nonalcoholic fatty liver (NAFL) and how such changes contribute to impaired health. Specifically, this thesis focusses on liver fat, with special attention for the composition of the fat, and hepatic glycogen. Fats in the liver are composed of glycerol and fatty acids and the fatty acids can be classified based on the number of double bonds in saturated, monounsaturated and poly-unsaturated fatty acids. In general, saturated fatty acids are believed to be harmful to your health. Glycogen is the stored form of glucose and the liver is an important organ for this storage. This stored form of glucose can be used as energy source by the body when needed, for example during fasting when the body is not getting glucose from food. Liver glycogen could play an important role in determining whether body fat will be used or stored. In this thesis, liver fat content and composition and liver glycogen are determined by magnetic resonance spectroscopy methodologies. Magnetic resonance spectroscopy is a non-invasive technique that can be used to study the metabolism of organs such as the liver, without the need for taking biopsies.

In chapter 2 the available methods to study the different routes that contribute to storage and disposal of fat in the liver were reviewed. It appeared that most studies are using techniques that require specific expertise and are costly. Therefore, the number of studies investigating the importance of these storage and disposal routes in the development of NAFL are limited, and even less is known about how diet can influence these routes.

One of the routes that can contribute to NAFL is the formation of fat from carbohydrates, called de novo lipogenesis. This newly formed fat is mainly saturated fat. In chapter 3, a new magnetic resonance spectroscopy technique was set-up to measure the fraction of the saturated, mono-unsaturated and poly-unsaturated fat in the liver. Once it was possible to measure these fat fractions in the liver, the relationship with de novo lipogenesis and liver insulin sensitivity could be studied. Liver insulin sensitivity indicates how well the liver responds to the hormone insulin, which is essential in maintaining normal blood sugar levels. It was shown that the higher de novo lipogenesis, the larger the fraction of saturated fat in the liver. Also, it was shown that in people with a large fraction of saturated fat in the liver, the sensitivity for insulin was reduced. A next step will be to investigate whether these relationships are causal. For example, does insulin sensitivity improve when we lower the amount of saturated fat in the liver by medication or diet? 
In chapter 4, it was investigated if extending the overnight fast by 6.5 hours has beneficial effects on the health of volunteers with NAFL. This was done by eating the last meal of the day at $4.30 \mathrm{pm}$ and comparing this to eating the last meal of the day at $11 \mathrm{pm}$. Extending fasting time for a few hours each day has been shown before to have beneficial health effects. This could be due to fluctuations in liver glycogen. From our results, it appeared that people with NAFL are not sensitive to an extended overnight fast as liver glycogen did not change. Normally, fat oxidation is increased with fasting, but also this we did not see in these volunteers with NAFL. Furthermore, when the volunteers continued to extend their overnight fast for 5 days, no effects were seen on the amount and composition of liver fat. But why is the effect of fasting disturbed in people with NAFL? New research has to be performed to answer this question, which can lead to novel insights to improve health.

Another possible way to improve health and liver fat storage is by diet. There are indications that the amount of saturated fat in the diet and the glycemic index of the diet can play an important role. The glycemic index (GI) indicates how quickly food can increase blood glucose levels; the higher the GI of food, the quicker the blood glucose level increases. In chapter $\mathbf{5}$ it was tested in overweight and obese volunteers whether a two-week diet low in saturated fat and GI would reduce liver fat storage when compared to a two-week diet high in saturated fat and GI. Indeed, it appeared that by only reducing the saturated fat and GI content of the diet for 2 weeks, liver fat storage decreased.

In addition to these results, it was shown in chapter 6 that factors related to the way of measuring liver fat content with magnetic resonance spectroscopy and the calculations used can have a big influence on the amount of liver fat content that is measured. In chapter 7, a supplement, nicotinamide riboside, was used to improve health of overweight and obese people using some of the techniques that were also applied in abovementioned studies. It appeared that some health aspects, such as body composition, were improved, but that liver fat content and insulin sensitivity were unchanged.

\section{What is the contribution of the results to science and societal challenges?}

Obesity rates are extremely high worldwide and NAFL is frequently present in people with obesity. NAFL is the most common cause of chronic liver disease and can progress to more severe liver disease, but is also often accompanied by type 2 diabetes and cardiovascular disease. At present, knowledge about liver metabolism in people with NAFL and the health consequences of disturbed liver metabolism are limited.

The results of the research described in this thesis contribute to our understanding of human liver metabolism and its importance for human health. Based on the results of this 
research new targets can be found that are important for improving health. Future studies can extend the findings presented in this thesis by investigating if lowering saturated fat in the liver and promoting fluctuations in liver glycogen by diet, exercise or medication can improve health of people with NAFL and related diseases, such as type 2 diabetes. Also, lowering dietary saturated fat and GI could be implemented by food industry, health care professionals, and government agencies in order to prevent the development of NAFL or treat NAFL and related diseases. Ultimately, this will contribute to reducing health care costs and relieving the pressure on the health care system.

\section{For whom are the results interesting and of relevance?}

The results and conclusions presented in this thesis are interesting for other researchers, who can set-up new studies further investigating the exact role of liver glycogen, saturated fat and de novo lipogenesis in NAFL and how these factors contribute to the development of NAFL related diseases. These studies can make use of the techniques described in this thesis, specifically the technique to specifically determine saturated fatty acids in the liver is new and enables us and other researchers in the field to perform follow-up studies. Ultimately, this knowledge could help in the prevention and treatment of NAFL and related diseases. In terms of prevention, this knowledge would be of interest for people with overweight and obesity, as these are at increased risk for developing NAFL. Specific lifestyle advice, such as minimizing dietary saturated fat and GI intake, may help this group at risk to keep liver fat content low and composition beneficial. Improving the quality of food products by the food industry in terms of saturated fat and Gl could also aid in the prevention of fatty liver development. Next to this, knowledge from future studies building on our results can lead to the development of new drugs for the treatment of NAFL, thereby reducing the risk for progressed liver disease and the development of related diseases such as type 2 diabetes in people with NAFL.

Other researchers will be informed about the results described in this thesis through publications in scientific journals and presentations at national and international conferences. Results will also be shared on websites, social media and participant information events, thereby informing the people at risk for the development of NAFL and the people with NAFL or related diseases. 


\section{SUMMARY}

Non-alcoholic fatty liver (NAFL) is the leading cause of chronic liver disease and highly prevalent in obesity. NAFL is defined as intrahepatic lipids (IHL) exceeding $5 \%$ of liver weight, in absence of excessive alcohol consumption. NAFL can progress to more severe stages of liver disease, such as non-alcoholic steatohepatitis (NASH) and liver cirrhosis, conditions in which liver function is severely hampered and the risk for hepatocellular carcinoma is increased. Importantly, NAFL per se is already very strongly associated with metabolic diseases such as cardiovascular disease (CVD) and type II diabetes (T2D). Knowledge on the metabolic changes that take place during the development of NAFL and how NAFL contributes to cardiometabolic disease is limited, particularly because of the limitations in techniques available. Thus, advanced methodologies are needed to investigate metabolic processes in NAFL. Magnetic resonance spectroscopy (MRS) can be applied to acquire chemical information of tissues, thereby making it a useful technique to study hepatic substrate stores. The research presented in this thesis describes the investigation of liver substrate metabolism in NAFL using advanced MR methodology.

While the pathways determining IHL content have long been identified (direct fat storage from a meal, de novo lipogenesis (DNL), uptake of plasma non-esterified fatty acids (NEFA), mitochondrial fatty acid oxidation, and secretion into the circulations within VLDLparticles), knowledge on the contribution of each of these pathways to IHL content in humans is sparse. Such knowledge is however essential in order to develop strategies to prevent and treat NAFL. Therefore, in chapter $\mathbf{2}$ we reviewed the techniques available to study the different pathways leading to $\mathrm{IHL}$ accumulation and the studies using these techniques to estimate the relative contribution of the different pathways or to study dietary modulation of these pathways.

Increased DNL may be one of the underlying pathways leading to NAFL. The end product of DNL are saturated fatty acids (SFA), and therefore high DNL rates might contribute to an increased hepatic SFA fraction. It is suggested that specifically these SFA, negatively influence metabolic health. Therefore, in chapter $\mathbf{3}$ a novel MR technique was developed, validated and applied that enabled to non-invasively quantify the fractions of hepatic SFA, mono-unsaturated fatty acids (MUFA) and poly-unsaturated fatty acids (PUFA) separately, in healthy and metabolically compromised human volunteers. Using this methodology, we showed in chapter 3 that DNL is positively associated with hepatic SFA fraction and is elevated in patients with NAFL and T2D. Furthermore, we showed that hepatic SFA fraction is strongly and negatively correlated with hepatic insulin sensitivity. These results suggest 
that hepatic SFA fraction is reflecting DNL and suggest that specifically the hepatic SFA fraction may hamper hepatic insulin sensitivity.

Another key factor in hepatic substrate metabolism could be hepatic glycogen stores. Hepatic glycogen is thought to change dynamically, decreasing during fasting and being replenished upon feeding. It is suggested that the regular depletion of hepatic glycogen stores is underlying the beneficial effects seen with time restricting eating regimes. To study the importance of hepatic glycogen stores in NAFL, prolonged overnight fasting was used as a tool to modulate hepatic glycogen in volunteers with NAFL in chapter 4 . We investigate if extending fasting time from $9.5 \mathrm{~h}$ after the last meal to $16 \mathrm{~h}$ after the last meal would acutely lower hepatic glycogen by using ${ }^{13} \mathrm{C}-\mathrm{MRS}$ and thereby improve substrate oxidation in volunteers with NAFL. We showed that prolonging the overnight fast does not affect hepatic glycogen content. Interestingly, a prolonged fast of $16 \mathrm{~h}$ did not result in higher fat oxidation rates during the night either, suggesting that these volunteers with NAFL did not fully reach a fasting state. Furthermore, prolonging the overnight fast for five days did not improve IHL content or composition. Altogether, these results suggest that hepatic substrate metabolism is not sensitive to extending acute fasting periods in individuals with NAFL.

A healthy diet is a cornerstone in the prevention and treatment of obesity and related metabolic conditions such as NAFL. Importantly, not only the amount of dietary fat and carbohydrate, but also their quality seems to be of significance. Specifically, high amounts of dietary SFA and carbohydrate-rich foods with a high glycemic index (GI), are thought to increase IHL, but human data is yet limited to proof of principle studies with exaggerated differences in diet composition. In chapter 5, it was investigated whether a two-week low vs. high GI/SFA diet, with similar macronutrient composition, reduces liver fat content and whether this is paralleled by increased whole-body fat oxidation, decreased glycemic response and reduced hepatic glycogen levels. To this end, overweight/obese volunteers participated in a randomized cross-over trial, following a two-week high GI/SFA diet and a two-week low GI/SFA diet with a wash-out period of 4 weeks. We showed that IHL was lower after a two-week low vs. high GI/SFA diet. Furthermore, the glycemic response tended to be reduced after the low vs. high GI/SFA diet and fat oxidation was increased early after the meal in the low vs. high GI/SFA diet. Hepatic glycogen content in the morning and whole-body substrate oxidation during the night were similar between the two interventions. These results indicate that realistic reductions in both GI and SFA content beneficially affect IHL in overweight/obese subjects. 
Quantification of IHL could be influenced by different factors and it is important to understand how these can bias IHL quantification. In chapter 6 it was evaluated how variations in $\mathrm{T} 2$ relaxation times affect the calculations of absolute $\mathrm{IHL} \%(\mathrm{w} / \mathrm{w})$ and to what extend the bias occurs while using different IHL quantification formulas. We showed that in individuals with different levels of steatosis, using fixed average T2 correction factors lead to an overestimation of IHL content in people with high IHL levels. However, by using STEAM sequence with short echo time the bias in IHL content can be minimized. Additionally, it was shown that the formula to express IHL is important, as it can introduce bias in individuals with high IHL levels.

High prevalence of overweight and obesity put large population groups at risk for NAFL and metabolic disease. Results from animal studies suggest that nicotamine riboside (NR), as an NAD+ precursor, can have metabolically beneficial effects, but human data is largely lacking. In chapter 7, the metabolic effect of six-week NR supplementation was assessed. We showed that NR supplementation increases skeletal muscle NAD+ metabolites, affects skeletal muscle acetylcarnitine metabolism, and induces minor changes in body composition and sleeping metabolic rate. No effects were observed in other measurements of metabolic health, including IHL stores and insulin sensitivity.

Overall, the studies described in this thesis investigated liver substrate metabolism in NAFL using advanced MR methodology, specifically focusing on the role of IHL composition and hepatic glycogen stores. From our studies it appears that in NAFL, hepatic SFA fraction is increased and hepatic glycogen cycling is reduced and furthermore, that high hepatic SFA fractions may hamper hepatic insulin sensitivity. Therefore, the hepatic SFA fraction and hepatic glycogen cycling could be interesting novel targets in the prevention and treatment of NAFL and other metabolic diseases. 


\section{SAMENVATTING}

Niet-alcoholische vette lever (NAFL) is de belangrijkste oorzaak van chronische leverziekte en komt veel voor bij obesitas. NAFL wordt gedefinieerd als intrahepatische lipiden (IHL) van meer dan $5 \%$ van het levergewicht, zonder aanwezigheid van overmatig alcoholgebruik. NAFL kan ontwikkelen naar ernstigere stadia van leverziekte, zoals niet-alcoholische steatohepatitis (NASH) en levercirrose, aandoeningen waarbij de leverfunctie ernstig wordt belemmerd en het risico op hepatocellulair carcinoom toeneemt. Belangrijk is dat NAFL op zich al zeer sterk geassocieerd is met metabole ziekten zoals hart- en vaatziekten (CVD) en type II-diabetes (T2D). De kennis over de metabole veranderingen die plaatsvinden tijdens de ontwikkeling van NAFL en hoe NAFL bijdraagt tot cardiometabole ziekten is beperkt, vooral door beperkingen in de beschikbare technieken. Daarom zijn geavanceerde methodologieën nodig om metabole processen in NAFL te onderzoeken. Magnetische resonantie spectroscopie (MRS) kan worden toegepast om chemische informatie van weefsels te verkrijgen, waardoor het een bruikbare techniek is om substraatvoorraden in de lever te bestuderen. In dit proefschrift wordt het onderzoek naar het substraatmetabolisme in de lever bij NAFL met behulp van geavanceerde MR-methodologie beschreven.

Hoewel de routes die het IHL-gehalte bepalen al lang zijn geïdentificeerd (directe vetopslag na de maaltijd, de novo lipogenese (DNL), opname van vrije vetzuren (NEFA), mitochondriale vetoxidatie, en secretie in de circulatie in VLDL-deeltjes), is de kennis over de bijdrage van elk van deze routes aan het IHL-gehalte in de mens schaars. Dergelijke kennis is echter essentieel voor het ontwikkelen van strategieën om NAFL te voorkomen en te behandelen. Daarom hebben we in hoofdstuk 2 een overzicht gegeven van de technieken die beschikbaar zijn om de verschillende routes die leiden tot IHL ophoping te bestuderen en van de studies die deze technieken gebruiken om de relatieve bijdrage van de verschillende routes te schatten of om de modulatie van deze routes door voeding te bestuderen.

Verhoogde DNL kan een van de onderliggende pathways zijn die tot NAFL leidt. Het eindproduct van DNL zijn verzadigde vetzuren (SFA), en daarom zouden hoge DNL-waarden kunnen bijdragen aan een verhoogde SFA fractie in de lever. Er wordt gesuggereerd dat juist deze SFA, een negatieve invloed hebben op de metabole gezondheid. Daarom werd in hoofdstuk 3 een nieuwe MR techniek ontwikkeld, gevalideerd en toegepast die het mogelijk maakt om op een niet-invasieve manier de fracties van SFA, enkelvoudig onverzadigde vetzuren (MUFA) en meervoudig onverzadigde vetzuren (PUFA) afzonderlijk in de lever te kwantificeren, in gezonde en metabool gecompromitteerde vrijwilligers. Met behulp van 
deze methodologie hebben wij in hoofdstuk 3 aangetoond dat DNL positief geassocieerd is met de lever SFA fractie en verhoogd is bij patiënten met NAFL en T2D. Verder hebben we aangetoond dat de lever SFA fractie sterk en negatief gecorreleerd is met insulinegevoeligheid van de lever. Deze resultaten suggereren dat de lever SFA fractie een afspiegeling is van DNL en suggereren dat specifiek de lever SFA fractie de insulinegevoeligheid van de lever kan belemmeren.

Een andere belangrijke factor in het levermetabolisme zou de leverglycogeenvoorraad kunnen zijn. Aangenomen wordt dat leverglycogeen dynamisch verandert, afneemt tijdens het vasten en weer wordt aangevuld bij het eten. Er wordt gesuggereerd dat de regelmatige uitputting van de leverglycogeenvoorraden ten grondslag ligt aan de gunstige effecten die worden waargenomen bij 'time-restricted eating'. Om het belang van leverglycogeenvoorraden in NAFL te bestuderen, is in hoofdstuk 4 langdurig vasten gedurende de nacht gebruikt als een middel om leverglycogeen in vrijwilligers met NAFL te moduleren. We hebben met behulp van ${ }^{13} \mathrm{C}-\mathrm{MRS}$ onderzocht of het verlengen van de vastentijd van 9.5 uur na de laatste maaltijd tot 16 uur na de laatste maaltijd het leverglycogeen acuut zou verlagen en daarmee de substraatoxidatie zou verbeteren in vrijwilligers met NAFL. Wij toonden aan dat het verlengen van de gevaste tijd geen invloed heeft op het leverglycogeengehalte. Interessant is dat het verlengde vasten van 16 uur ook niet resulteerde in een hogere vetoxidatie gedurende de nacht, wat suggereert dat deze vrijwilligers met NAFL geen volledig gevaste toestand bereikten. Bovendien leidde het verlengd nachtelijke vasten gedurende vijf dagen niet tot een verbetering van het gehalte of de samenstelling van het IHL. Al met al suggereren deze resultaten dat het levermetabolisme niet gevoelig is voor het verlengen van de acute vastenperiode bij mensen met NAFL.

Een gezond dieet is een belangrijke pijler in de preventie en behandeling van obesitas en gerelateerde metabole aandoeningen zoals NAFL. Niet alleen de hoeveelheid vetten en koolhydraten in de voeding, maar ook de kwaliteit ervan lijkt belangrijk te zijn. Hoge hoeveelheden SFA in de voeding en koolhydraatrijke voedingsmiddelen met een hoge glycemische index (GI), zouden de IHL verhogen, maar humane data zijn nog beperkt tot 'proof of principle' studies met overdreven verschillen in voedingssamenstelling. In hoofdstuk 5 werd onderzocht of een twee weken durend laag vs. hoog GI/SFA dieet, met vergelijkbare macronutriënt samenstelling, het vetgehalte in de lever verlaagt en of dit gepaard gaat met een verhoogde vetoxidatie, een verlaagde glycemische respons en verlaagde leverglycogeenniveaus. Hiertoe namen vrijwilligers met overgewicht of obesitas deel aan een gerandomiseerde cross-over studie, waarbij ze twee weken een hoog GI/SFA dieet volgden en twee weken een laag GI/SFA dieet met een wash-out periode van 4 weken. 
Wij toonden aan dat het IHL lager was na een twee weken durend laag versus hoog GI/SFA dieet. Bovendien was de glycemische respons lager na het lage vs. hoge GI/SFA dieet en was de vetoxidatie kort na de maaltijd verhoogd bij het lage vs. hoge GI/SFA dieet. Het hepatische glycogeengehalte in de ochtend en de substraatoxidatie gedurende de nacht waren vergelijkbaar tussen de twee interventies. Deze resultaten wijzen erop dat realistische verlagingen van zowel de GI als de hoeveelheid SFA een gunstig effect hebben op het IHL bij mensen met overgewicht of obesitas.

Kwantificering van het IHL kan beïnvloed worden door verschillende factoren en het is belangrijk om te begrijpen hoe deze de IHL kwantificering kunnen vertekenen. In hoofdstuk 6 is onderzocht hoe variaties in T2 relaxatietijden de berekeningen van het absolute IHL\% $(w / w)$ beïnvloeden en in hoeverre de bias optreedt bij gebruik van verschillende IHL kwantificeringsformules. We toonden aan dat bij personen met verschillende niveaus van steatose het gebruik van vaste gemiddelde T2-correctiefactoren leidt tot een overschatting van het IHL-gehalte bij personen met een hoog IHL-gehalte. Door gebruik te maken van een STEAM sequentie met korte echotijd kan de bias in het IHL gehalte echter geminimaliseerd worden. Bovendien werd aangetoond dat de formule om IHL uit te drukken belangrijk is, aangezien deze een bias kan introduceren bij personen met een hoog IHL gehalte.

Door de hoge prevalentie van overgewicht en obesitas loopt een groot deel van de bevolking een risico op NAFL en metabole ziekten. Resultaten van dierstudies suggereren dat nicotamine riboside (NR), als een NAD+ precursor, metabool gunstige effecten kan hebben, maar informatie over de effecten in de mens ontbreken grotendeels. In hoofdstuk 7 werd het metabole effect van zes weken NR suppletie onderzocht. We toonden aan dat NR-suppletie de NAD+ metabolieten in de skeletspieren verhoogt, het acetylcarnitine metabolisme in de skeletspieren beïnvloedt, en kleine veranderingen in lichaamssamenstelling en slaapmetabolisme induceert. $\mathrm{Er}$ werden geen effecten waargenomen in andere metingen van metabole gezondheid, waaronder IHL-voorraden en insulinegevoeligheid.

In het algemeen hebben de in dit proefschrift beschreven studies het substraatmetabolisme van de lever bij NAFL onderzocht met behulp van geavanceerde MR-methodologie, waarbij de nadruk lag op de rol van de IHL-samenstelling en de leverglycogeenvoorraden. Uit onze studies blijkt dat bij NAFL de SFA fractie in de lever verhoogd en de leverglycogeencyclus verstoord is, en bovendien dat hoge SFA leverfracties de leverinsulinegevoeligheid kunnen belemmeren. Daarom zouden de lever SFA fractie en het lever glycogeen interessante nieuwe doelen kunnen zijn in de preventie en behandeling van NAFL en andere metabole ziekten. 


\section{ABOUT THE AUTHOR}

Kay Roumans was born on January 14th 1992 in Meerssen, the Netherlands. After finishing secondary education at Stella Maris Meerssen in 2010, Kay started his study Biomedical sciences at Maastricht University. During his master he performed internships at the department of Human Biology, investigating the effect of bile acid supplementation on brown adipose tissue activity and energy expenditure, and studying the relation between skeletal muscle mitochondrial function and hepatic lipid accumulation in people with compromised metabolic health. In 2015, he completed his master's in biomedical

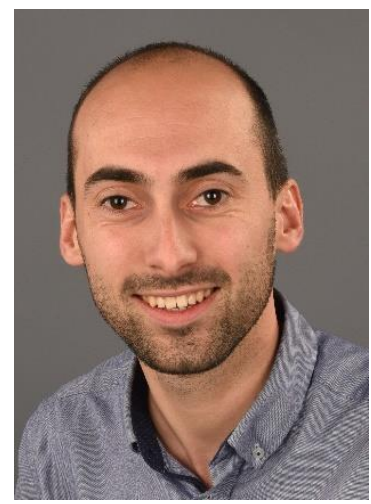
sciences, with a specialization in Nutrition and Metabolism. In 2016, he started his PhD at Maastricht University at the department of Nutrition and Movement Sciences under supervision of prof. dr. Patrick Schrauwen, dr. Vera Schrauwen-Hinderling and dr. ir. Lucas Lindeboom. During his PhD he worked on the topic of substrate metabolism in people with NAFL within a TKI/Health Holland consortium with partners from Leiden University Medical Center and Unilever R\&D. In this human research he applied both invasive techniques, such as hyperinsulinemic-euglycemic clamps, and non-invasive techniques, such as Magnetic Resonance Spectroscopy. Kay will continue his career as a postdoctoral researcher at the department of Nutrition and Movement Sciences and will perform a human intervention study to further unravel the role of de novo lipogenesis and hepatic saturated fatty acids in insulin resistance. 


\section{LIST OF PUBLICATIONS}

1. K.H.M. Roumans*, L. Lindeboom*, P. Veeraiah, C.M.E. Remie, E. Phielix, B. Havekes, Y.M.H. Bruls, M.C.G.J. Brouwers, M. StåhIman, M. Alssema, H.P.F. Peters, R. de Mutsert, B. Staels, M-R. Taskinen, J. Borén, P. Schrauwen, V. B. Schrauwen-Hinderling. Hepatic saturated fatty acid fraction is associated with de novo lipogenesis and hepatic insulin resistance. Nat. Comm. 2020 Apr 20;11(1):1891. doi: 10.1038/s41467-020-15684-0.

2. K.H.M. Roumans, J. Basset, H.P.F. Peters, P. Schrauwen, V.B. Schrauwen-Hinderling. Liver fat storage pathways: methodologies and dietary effects. Curr Opin Lipidol. 2021 Feb 1;32(1):9-15. doi: 10.1097/MOL.0000000000000720.

3. C.M.E. Remie, K.H.M. Roumans, M.P.B. Moonen, N.J. Connell, B. Havekes, J. Mevenkamp, L. Lindeboom, V.H.W. de Wit, T. van de Weijer, S.A.B.M. Aarts, E. Lutgens, B.V. Schomakers, H. L. Elfrink, R. Zapata-Pérez, R.H. Houtkooper, J. Hoeks, V.B. Schrauwen-Hinderling, E. Phielix, P. Schrauwen. A randomized placebo-controlled clinical trial of nicotinamide riboside supplementation in healthy obese humans: effects on metabolic health. Am J Clin Nutr. 2020 Aug 1;112(2):413-426. doi: 10.1093/ajcn/nqaa072/5823793

4. C.M.E. Remie*, M.P.B. Moonen*, K.H.M. Roumans, Emmani B.M. Nasimento, Anne Gemmink, B. Havekes, G. Schaart, E. Kornips, P.J. Joris, V.B. Schrauwen-Hinderling, J. Hoeks, S. Kersten, M.K.C. Hesselink, E. Phielix, W.D. van Marken Lichtenbelt, P. Schrauwen. Metabolic responses to mild cold acclimation in type 2 diabetes patients. Nat Commun 12, 1516 (2021). https://doi.org/10.1038/s41467-021-21813-0

5. E. van Eekelen, I. Verkouter, H.P.F. Peters, M. Alssema, B.G. de Roos, V.B. SchrauwenHinderling, K.H.M. Roumans, J.W. Schoones, P.L. Zock, P. Schrauwen, F.R. Rosendaal, O.M. Dekkers, and R. de Mutsert. Effects of dietary macronutrients on liver fat content in adults: a systematic review and meta-analysis of randomized controlled trials. Eur J Clin Nutr. 2020 Oct 22. doi: 10.1038/s41430-020-00778-1.

6. Broeders EP, Nascimento EB, Havekes B, Brans B, Roumans KH, Tailleux A, Schaart G, Kouach M, Charton J, Deprez B, Bouvy ND, Mottaghy F, Staels B, van Marken Lichtenbelt WD, Schrauwen P. The Bile Acid Chenodeoxycholic Acid Increases Human Brown Adipose Tissue Activity. Cell Metab. 2015 Sep 1;22(3):418-26. doi: 10.1016/j.cmet.2015.07.002. 


\section{DANKWOORD}

Dan rest mij nog om iedereen te bedanken die de totstandkoming van mijn proefschrift mede mogelijk heeft gemaakt. Ik zal vast vergeten een aantal mensen te bedanken, maar zij hebben hopelijk de afgelopen jaren ook al van mij gehoord dat ik ze dankbaar ben!

Mijn promotieteam: Patrick, Vera en Lucas. Dank voor jullie prettige begeleiding. Er is toch heel wat tijd door jullie gestoken in o.a. meetings en het nakijken van manuscripten. Vera, ik wil jou in het bijzonder bedanken. Jij hebt de dagelijkse begeleiding op je genomen en wekelijks de tijd genomen om dingen met mij te bespreken. Ik vond onze meetings altijd heel luchtig en effectief, dat heb ik altijd erg gewaardeerd.

Dank aan iedereen van het TKI Health-Holland consortium. Het was heel fijn met jullie samenwerken en zo veel van jullie te leren. Ik vond het altijd mooi om te zien hoe iedereen vanuit verschillende invalshoeken ons project nog interessanter kon maken.

Ik wil alle DMRG en VBW collega's bedanken waarmee ik de afgelopen jaren heb samengewerkt. Dankzij jullie ging alles toch net wat gemakkelijker en waren die vroege ochtenden en overnachtingen veel beter vol te houden. Een speciaal woord van dank aan Jeremy. Jij hebt een groot deel van de voedingsstudie op je genomen. Testdagen zijn altijd een groot feest met jou! Ook wil ik Pandi, Julian en Kim bedanken voor alle MRSgerelateerde hulp. Zonder jullie had ik het niet gekund. Dan nog een speciaal woord van dank aan mijn inmiddels oud-collega's 'achter in de gang': Bas, Carlijn, Yvo, Vera. De afgelopen jaren heb ik mijn collega's ook als vrienden gezien, maar jullie zijn meer dan gewoon vrienden. Alles kon ik met jullie delen: leuke verhalen, nieuwtjes, maar ook zorgen en problemen. Ook niet onbelangrijk waren de lekkernijen die we deelden, zoals de vlaai, snoep, chocola etc... Qua gezondheid ben ik er de afgelopen jaren vast niet op vooruit gegaan.

Audrey, jou bedank ik als allerlaatst. Het is met geen woorden te beschrijven hoe veel jij me steunt. Dat ga ik dan ook niet proberen, je weet hoe dankbaar ik je daar voor ben! 
